\title{
METEOROLOGICAL, STREAM-DISCHARGE, AND WATER-QUALITY DATA FOR WATER YEAR 1992 FROM TWO BASINS IN CENTRAL NEVADA
}

\section{by Patrick W. McKinley and Thomas A. Oliver}

\section{U.S. GEOLOGICAL SURVEY}

Open-File Report 94-456

Prepared in cooperation with the

NEVADA OPERATIONS OFFICE,

U.S. DEPARTMENT OF ENERGY under

Interagency Agreement DE-Al08-92NV10874

\section{DISCLAIMER}

This report was prepared as an account of work sponsored by an agency of the United States Government. Neither the United States Government nor any agency thereof, nor any of their employees, makes any warranty, express or implied, or assumes any legal liability or responsibility for the accuracy, completeness, or usefulness of any information, apparatus, product, or process disclosed, or represents that its use would not infringe privately owned rights. Reference herein to any specific commercial product, process, or service by trade name, trademark, manufacturer, or otherwise does not necessarily constitute or imply its endorsement, recommendation, or favoring by the United States Government or any agency thereof. The views and opinions of authors expressed herein do not necessarily state or reflect those of the United States Government or any agency thereof.

Denver, Colorado

1995

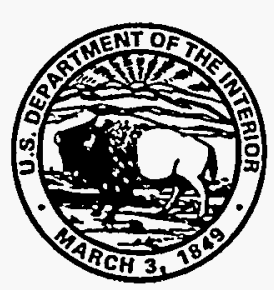




\title{
U.S. DEPARTMENT OF THE INTERIOR \\ BRUCE BABBITT, Secretary
}

\author{
U.S. GEOLOGICAL SURVEY
}

Gordon P. Eaton, Director

The use of trade, product, industry, or firm names is for descriptive purposes only and does not imply endorsement by the U.S. Government.

For additional information write to: Chief, Earth Science Investigations Program Yucca Mountain Project Branch U.S. Geological Survey Box 25046, MS 421 Denver Federal Center Denver, CO 80225
Copies of this report can be purchased from: U.S. Geological Survey Earth Science Information Center Open-File Reports Section Box 25286, MS 517 Denver Federal Center Denver, CO 80225 


\section{DISCLAIMER}

Portions of this document may be illegible in electronic image products. Images are produced from the best available original document. 


\section{CONTENTS}

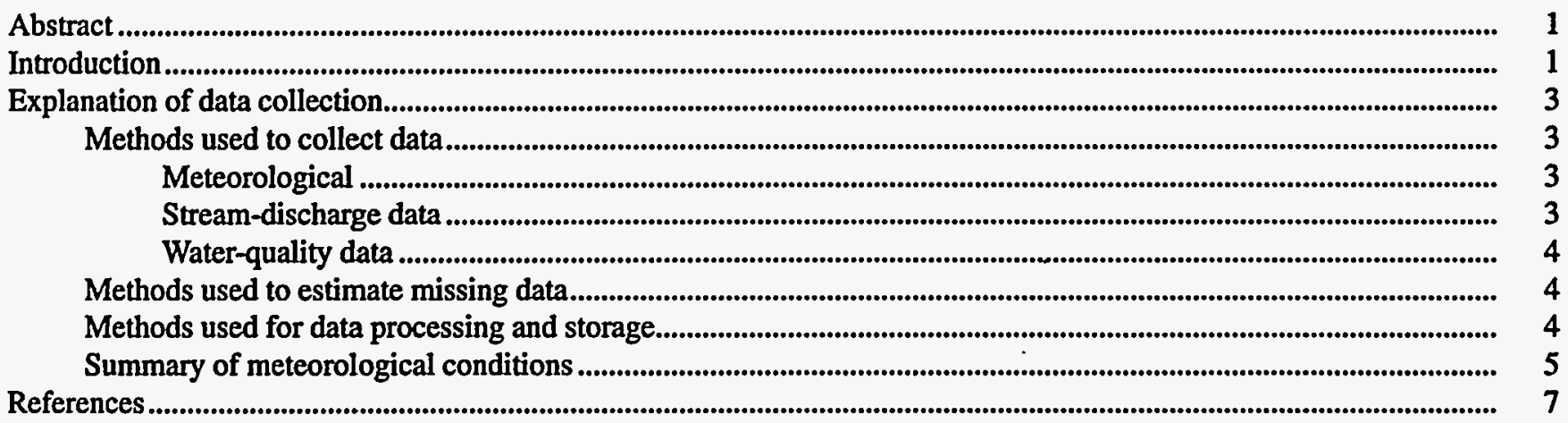

\section{DATA COLLECTION STATIONS FOR WHICH RECORDS ARE INCLUDED IN THIS REPORT}

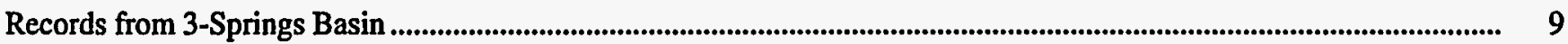

Kawich Peak near Warm Springs, Nevada .......................................................................................................................... 10

Daily mean air temperature for 1991-1992 _............................................................................................................. 11

Daily maximum air temperature for 1991-1992 ........................................................................................... 12

Daily minimum air temperature for 1991-1992 .............................................................................................. 13

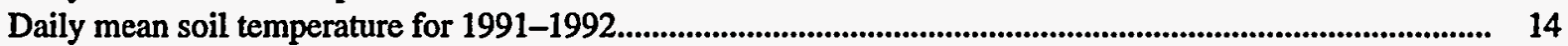

Daily total solar radiation, incidental for 1991-1992 ........................................................................................... 15

Daily mean relative humidity for 1991-1992 .................................................................................................. 16

Daily total precipitation for 1991-1992 ..................................................................................................................... 17

Precipitation water quality for 1991-1992.................................................................................................... 18

3-Springs Creek near Ledge Spring, near Warm Springs, Nevada .................................................................................... 19

Surface-water quality for 1991-1992 ..................................................................................................................... 19

3-Springs Creek near 3-Spring \#2, near Warm Springs, Nevada...................................................................................... 20

Surface-water quality for 1991-1992 ............................................................................................................................... 20

3-Springs Creek near Warm Springs, Nevada.......................................................................................................... 21

Daily mean air temperature for 1991-1992 ......................................................................................... 22

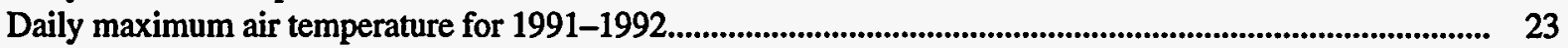

Daily minimum air temperature for 1991-1992 ............................................................................................. 24

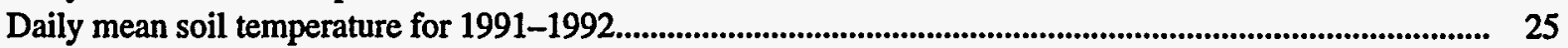

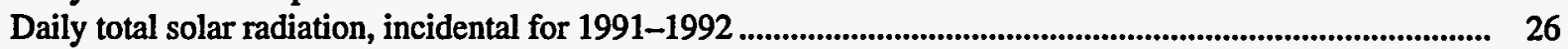

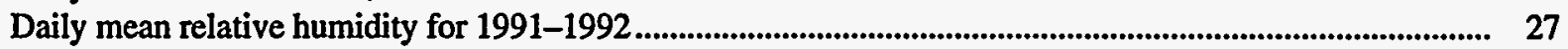

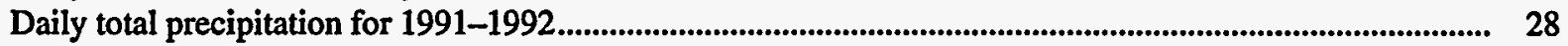

Precipitation water quality for 1991-1992 ............................................................................................................. 29

Water-quality analysis from the unsaturated-zone lysimeter for 1991-1992 ..................................................... 30

Daily mean discharge for 1991-1992 ................................................................................................... 31

Surface-water quality for 1991-1992 ...................................................................................................................... 32

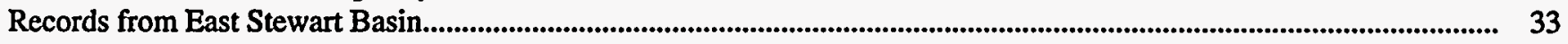

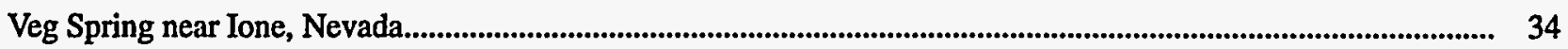

Daily mean air temperature for $1991-1992$............................................................................................................. 35

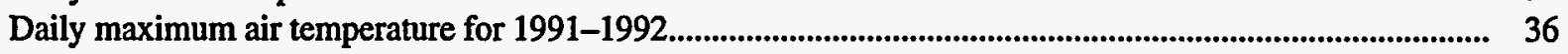

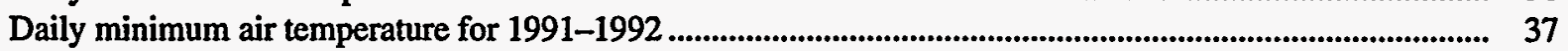

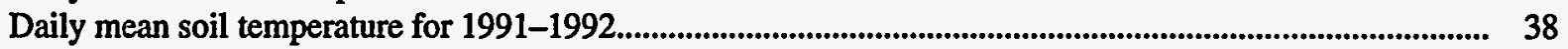

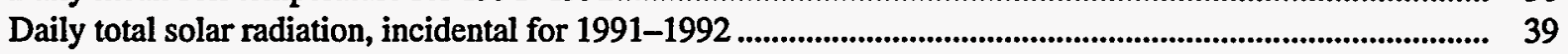

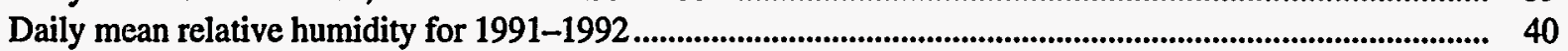

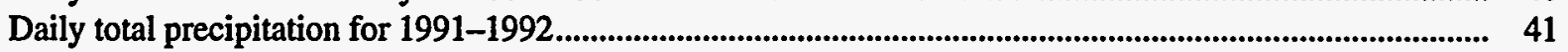

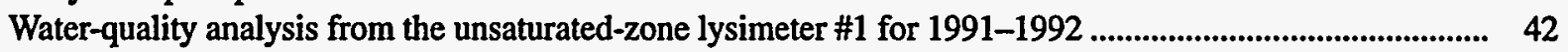

Surface-water quality for 1991-1992 ................................................................................................................. 43 


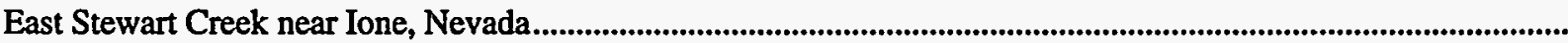

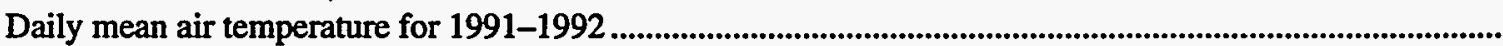

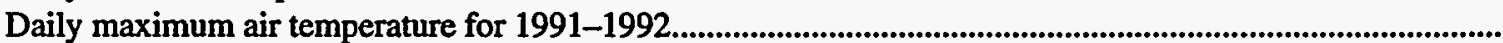

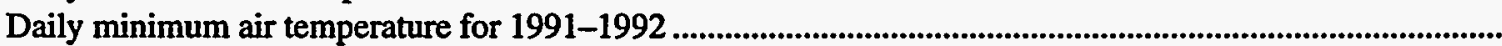

Daily mean soil temperature for 1991-1992..

Daily total solar radiation, incidental for 1991-1992 _........................................................................................... 49

Daily mean relative humidity for 1991-1992 ….................................................................................. 50

Daily total precipitation for 1991-1992 ...................................................................................................... 51

Precipitation water quality for 1991-1992 ..................................................................................................... 52

Water-quality analysis from the unsaturated-zone lysimeter for 1991-1992 .................................................... 53

Daily mean discharge for 1991-1992 ......................................................................................................................... 54

Surface-water quality for 1991-1992 .......................................................................................................... 55

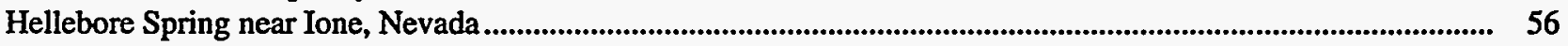

Surface-water quality for 1991-1992 ................................................................................................... 56

\section{FIGURES}

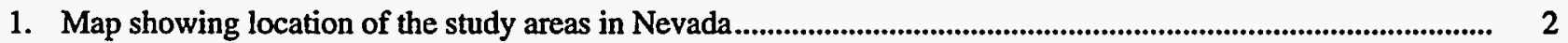

2. Boxplots of annual air temperature and annual precipitation at Austin, Nevada..................................................... 6

\section{TABLE}

1. 3-Springs and East Stewart Basin statistics

\section{CONVERSION FACTORS AND VERTICAL DATUM}

\begin{tabular}{rll}
\hline Multiply & By & To obtain \\
\hline & & \\
cubic foot per second $\left(\mathrm{ft}^{3} / \mathrm{s}\right)$ & 0.02832 & cubic meter per second \\
calorie $(\mathrm{cal})$ & 4.184 & joule \\
foot $(\mathrm{ft})$ & 0.3048 & meter \\
inch (in.) & 2.54 & centimeter \\
mile $(\mathrm{mi})$ & 1.609 & kilometer \\
square foot $\left(\mathrm{ft}^{2}\right)$ & 0.09294 & square meter \\
square mile $\left(\mathrm{mi}^{2}\right)$ & 2.59 & square kilometer \\
\hline
\end{tabular}

Degree Celsius $\left({ }^{\circ} \mathrm{C}\right)$ may be converted to degree Fahrenheit $\left({ }^{\circ} \mathrm{F}\right)$ by using the following equation:

$$
{ }^{\circ} \mathrm{F}=9 / 5\left({ }^{\circ} \mathrm{C}\right)+32 \text {. }
$$

Sea level: In this report "sea level" refers to the National Geodetic Vertical Datum of 1929 (NGVD of 1929)-a geodetic datum derived from a general adjustment of the first-order level nets of both the United States and Canada, formerly called Sea Level Datum of 1929. 


\title{
Meteorological, Stream-Discharge, and Water-Quality Data for Water Year 1992 from Two Basins in Central Nevada
}

\author{
ByPatrick W. McKinley andThomas A. Oliver
}

\section{Abstract}

Two small basins, measuring less than 2 square miles, were studied to determine the volume of precipitation available for recharge to ground water. The semiarid 3-Springs Basin is located to the east of Kawich Peak in the Kawich Range east of Tonopah, Nevada. Stewart Basin is a subalpine drainage basin north of Arc Dome in the Toiyabe Range north of Tonopah, Nevada. The purpose of this publication is to make available the meteorological, stream-discharge, and water-quality data collected during the study. Meteorological data collected include air temperature, soil temperature, solar radiation, and relative humidity. Stream-discharge data were collected from the surface-water outlet of each basin. Water-quality data are chemical analyses of water samples collected from surface- and ground-water sources.

Each basin has a meteorological station located in the lower and upper reaches of the basin. Hydrologic records include stream-discharge and water-quality data from the lower meteorological site and water-quality data from springs within the basins.

\section{INTRODUCTION}

The purpose of this report is to present the results of meteorological, stream-discharge, and water-quality measurements from two study basins in central Nevada. This is the second report of data from these basins; the first report covered the years 1986 through 1991. Collection techniques, locations, data processing, and presentation are the same as in the previous report but are restated for completeness and because the explanations are brief. A statistical representation of the study data collected from 1986 through 1992 and long term weather at Austin, Nevada, are compared to provide a means of evaluating the normality of the study period. The previous report did not contain an evaluation of the data normality as compared to a longterm record.

The U.S. Geological Survey, in cooperation with the U.S. Department of Energy, is studying Yucca Mountain, Nevada, as a potential repository for high level nuclear waste. As part of the Yucca Mountain Site Project, the analog recharge study is providing data for the evaluation of recharge to the Yucca Mountain ground-water system given a cooler and wetter climate than currently exists. The current arid climatic conditions are favorable to the isolation of radioactive waste. Because waste isolation from the accessible environment for 10,000 years is necessary, climatic change and the potential for increased ground-water recharge need to be considered as part of the characterization of the potential repository.

Two small basins, 3-Springs and East Stewart Basin, are similar geologically to the recharge area for Yucca Mountain. The 3-Springs Basin is in the Kawich Range east of Kawich Peak (fig. 1). Some basin statistics are listed in table 1. Vegetation is dominated by a pinon-juniper forest, but includes large areas of willows, mountain mahogany, and sage. The 3-Springs Creek is an intermittent creek at the basin outlet and is occasionally dry for the whole year. The major source of recharge is spring runoff; although, occasionally summer rain or heavy fall snowmelt may be sufficient to cause the creek to flow. 


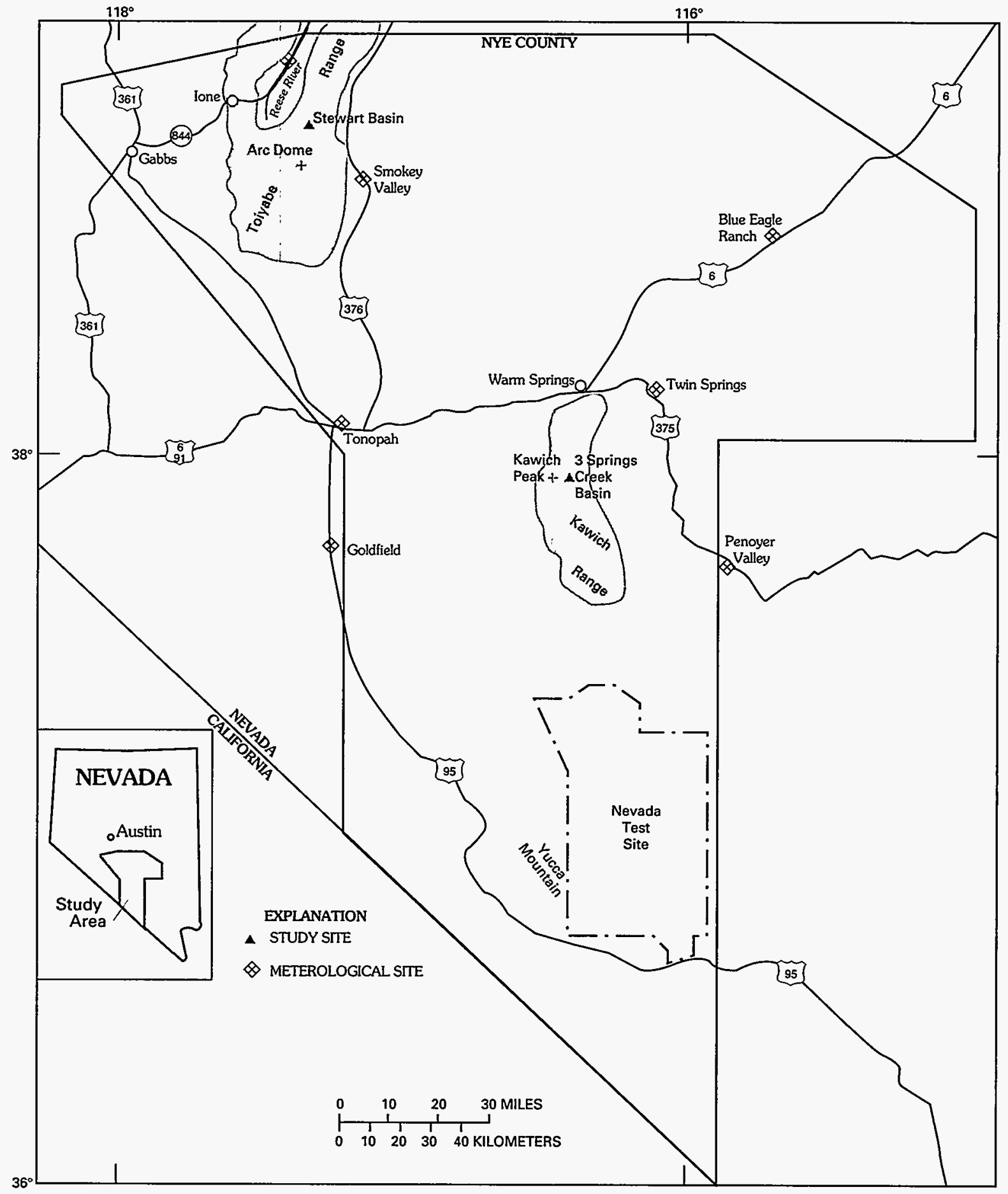

Figure 1. Location of the study areas in Nevada. 
Table 1. 3-Springs and East Stewart Basin statistics

(Locations and altitudes were taken from USGS 7 1/2" Kawich Peak NW, Nevada, provisional edition 1987, and $71 / 2^{n}$ South Toiyabe Peak, Nevada, 1979; mi $^{2}$, square miles; fh, feet]

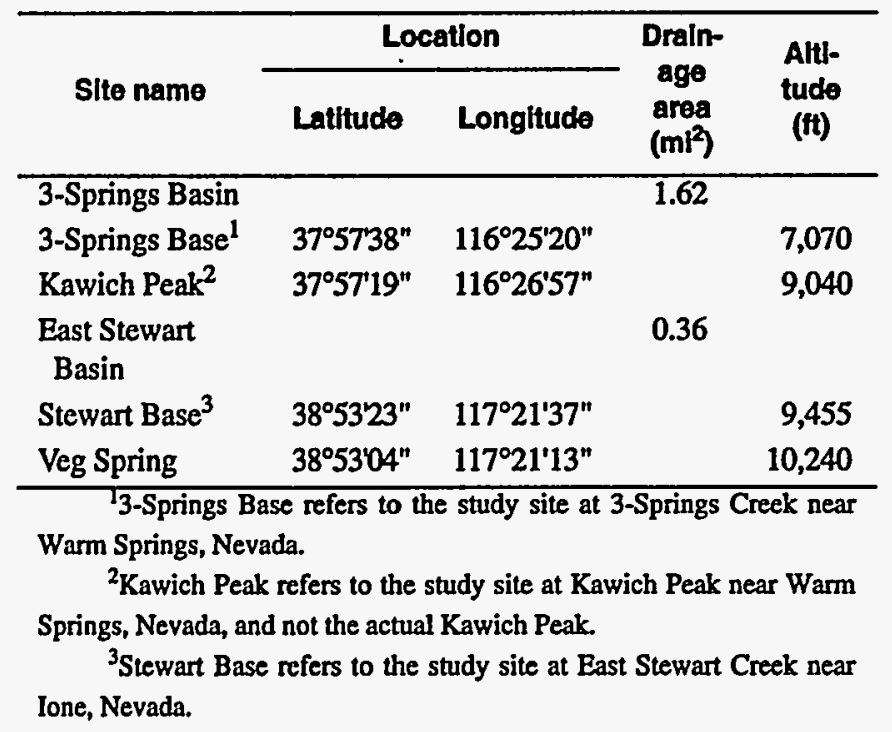

East Stewart Basin is in the Toiyabe Range north of Arc Dome (fig. 1). A subalpine forest containing aspen, mountain mahogany, and limber pine covers most of the basin. East Stewart Basin has a perennial stream that is fed by snowmelt from April through early to mid-June and by springs during the remainder of the year.

\section{EXPLANATION OF DATA COLLECTION}

\section{Methods Used to Collect Data}

\section{Meteorological}

Meteorological data were measured using Campbell Scientific Instrument Co., CSI, electronic dataloggers and the following probes: air temperature and relative humidity were measured by CSI 207 humidity probes, soil temperature was measured by CSI 107 temperature probes, and solar radiation was measured by Li-Cor Co. LI200SB probes.

Belfort weighing rain gages with Alter windshields were used to measure precipitation. Gages were mounted on adjustable platforms so they could be kept above the snow level. Precipitation was trapped by a film of oil to decrease evaporation in the summer. During the winter, an antifreeze solution was used to melt and retain the snowfall. A strip chart recorded time and amount of precipitation. Two gages were located in each basin - one at a low altitude in the basin and one in the higher part of the basin. The gage locations and altitudes are listed in table 1.

Air temperature, soil temperature, and relative humidity were measured every 30 seconds and averaged over a 24 -hour period. Solar radiation was measured over a 30-second interval and was totaled for a 24-hour period. Air temperature, soil temperature, and relative humidity are reported as daily average values. Precipitation and solar radiation are reported as daily total values. Calibrations of the meteorological measurement probes were at regular intervals and when possible, were field checked during each site visit. The CSI 107 temperature probes used to measure soil temperature were stable; probe measurements rarely varying more than $0.2^{\circ} \mathrm{C}$ from the calibration standard.

Relative humidity values reported are considered poor because the annual calibrations were generally off the standard by approximately 10 percent. Manufacturer specifications for the CSI 207 humidity probe indicated the probe was reliable between 15 through 85 percent relative humidity. The temperature measurements by the CSI 207 humidity probe were equivalent to the CSI 107 probe measurements discussed above. The LI200SB probes used for solar radiation measurements were calibrated every two years; the manufacture estimated a decline in sensitivity of about two percent per year. Radiation values were not corrected for the sensitivity decline. Calibration of rain gages was checked in the field annually using Belfort calibration weights. Rain gage accuracy was approximately 0.5 percent of full scale. More information about the measurements can be found in the Remarks section immediately preceding records for each site.

\section{Stream-Discharge Data}

Creek discharge was monitored using a V-notch weir and a Parshall flume. The gage height of the weir pool was monitored by a datalogger connected to a potentiometer-stilling well system. Gage heights were read by the datalogger every 30 seconds and averaged over a 30-minute period. In addition to the weir, 3-Springs Creek was equipped with a 6-in fiume and Stewart Creek with a 9-in flume. Because 3-Springs Creek is generally dry and low flows are significant to understanding the basin hydrology, discharge at 3-Springs Creek is expressed to three decimal places to indicate the presence of flow. The additional decimal place does not infer the record accuracy has increased. The water level in the flumes was monitored every 15 minutes by an automatic digital recorder and stilling well system. Additional information about the record 
is located in the Remarks section associated with the data tables at the back of the report.

\section{Water-Quality Data}

Precipitation samples taken during quarterly field trips were analyzed for major anions and cations; the stable isotopes, ${ }^{18} \mathrm{O}$ and deuterium; and the physical properties, $\mathrm{pH}$ and specific conductance. Concentrations of other elements and compounds were measured on an intermittent basis. Precipitation samples represent integrated soluble dry-plus-wetfall. The collection equipment and operation are described in Voegeli and Claassen (1971, p. 3-4). Surface-water samples were taken quarterly and analyzed for the same chemical constituents and properties as precipitation samples. Water temperatures reported for the surface-water samples were measured in the stream. Because of the small size of the streams, samples were dipped from the main flow of the stream and are referred to as grab samples.

Unsaturated-zone samples were taken from lysimeters located just below the root zone. Water samples were usually taken twice each year-one in the late spring, representing the infiltration resulting from snowmelt, and one in early fall representing summer rains. Springs also were sampled and were considered a representation of the shallow ground water. Chemical analysis consisted of major anions and cations; the stable isotopes ${ }^{18} \mathrm{O}$ and deuterium; and the physical properties, temperature, $\mathrm{pH}$, and specific conductance.

Samples generally were filtered within 24 hours of sampling. All samples then were analyzed at the USGS National Water-Quality Laboratory in Arvada, Colorado.

\section{Methods Used to Estimate Missing Data}

Estimating daily mean air and soil data was necessary only at Veg Spring near Ione. Temperatures were estimated by step-forward regression using data from the meteorological site at East Stewart Creek near Ione. Daily maximum and minimum air temperature and mean daily relative humidity were not estimated. Unrecoverable solar radiation data at Veg Spring was estimated by step-forward regression from the East Stewart Creek meteorological site data. For periods of less than a day, the Bird and Hulstrum (1981) clear sky model was used to calculate estimates. If only a few readings were missing, simple interpolation between known values was used. The use of the several meth- ods was necessary because there was no single method that provided the best estimate for all circumstances.

Two precipitation recorders in the East Stewart Basin enabled precipitation estimates to be made from data collected from within the immediate basin. Pen failure was the usual cause of lost precipitation record, but the amount of precipitation was available from the strip chart; only the timing of the precipitation was estimated. For example, humidity measurements from Veg Spring and precipitation records from East Stewart Creek were used to determine the timing of precipitation for the lost data at Veg Spring during the period June 12 through July 1. Estimated daily precipitation values for Veg Spring were calculated as noted in equation 1. The known daily precipitation at East Stewart Creek is $P, T a$ is the total precipitation at Veg Spring for the period to be estimated, and $T b$ is the total precipitation at East Stewart Creek for the total period to be estimated

$$
\text { estimated daily precipitation }=\frac{(P)(T a)}{T b}
$$

No surface-water estimates were necessary during the water year.

\section{Methods Used for Data Processing and Storage}

All time-sequence data were stored as unit values in the USGS National Water Information System (NWIS) data base, which provides calculation of daily, monthly, and annual means or totals. The NWIS software tabling routines were used to organize the data for this report. More information on the NWIS data base was reported by Dempster (1990).

Meteorological data, except for precipitation, were transferred electronically to NWIS and then processed by and stored in NWIS. Precipitation data were stored in NWIS, but the original data were recorded on strip charts and then manually translated and input into NWIS.

Surface-water-data computations were made according to methods described by Rantz (1982). Unit values, 30-minute average gage heights from the weir, and 15-minute instantaneous gage heights from the flume, were input into NWIS electronically. Datum and shift corrections were applied manually to the gage heights to correct for erroneous gage operation (datum) or for changes in the control (shift). Daily mean stage was computed by NWIS from the unit values; daily mean discharge was computed from the stagedischarge relation (rating curve) and the daily mean stage. 
The standard stage-discharge relation noted in equation 2, (U.S. Bureau of Reclamation, 1984),

$$
Q=2.49 H^{2.48}
$$

was used for conversion of stage to discharge from the $90^{\circ} \mathrm{V}$-notch thin-plate weirs. Where $Q$ is the discharge, in cubic feet per second, and $H$ is the stage, gage height, or head of the weir, in feet. Flume stagedischarge relations (ratings) were developed from actual laboratory tests (Dodge, 1990). Variations from standard flume dimensions and stream approach conditions accounted for the differences between the standard Parshall flume rating and the developed rating.

\section{Summary of Meteorological Conditions}

Weather records from Austin, Nevada, provide a 60-year meteorological setting (norm) to compare the 7-year study period. Austin weather data correlated better with data from the study sites than with data from other weather stations in central Nevada. For periods prior to 1992, when data were not available from a second site within the study basins, Austin weather data were used in estimating missing data at the study sites. Data for Austin were supplied by the Western Regional Climate Center in Reno, Nevada.

The boxplots in figure 2 show the annual air temperature median for the study period, 1986 through 1992 , to be as much as $0.8^{\circ} \mathrm{C}$ warmer than the 60 -year median and above the upper quartile of the 60 -year plot. During the period 1986 through 1992, monthly median air temperatures indicate that March and April were consistently above the long term monthly median for those months and were a major contributor to the annual air-temperature increase for the period. No correlation was found between median monthly air temperatures and total monthly precipitation at Austin for the period.

The boxplots in figure 2 of total annual precipitation show the precipitation median for the study period is the same as the 60- and 40-year medians. Total monthly precipitation at Austin for November 1986 and May 1987 was the maximum of record for those months; May 1987 precipitation was 5.85 in, 0.11 in less than the maximum monthly precipitation record. The precipitation at the study site was also above normal for these months and maximum of record at East Stewart Basin for May 1987.

From the above analysis, a general assessment of the period of record 1986 through 1992 is that the median annual air temperature was warmer than normal, and the total annual precipitation was about normal. There were no new annual extremes of record during the study period, but precipitation exceeded the upper quartile and the lower quartile of the 60 - and 40-year boxplots. Median annual air temperature also showed a wide range but was generally above the 60 - and 40-year medians. 

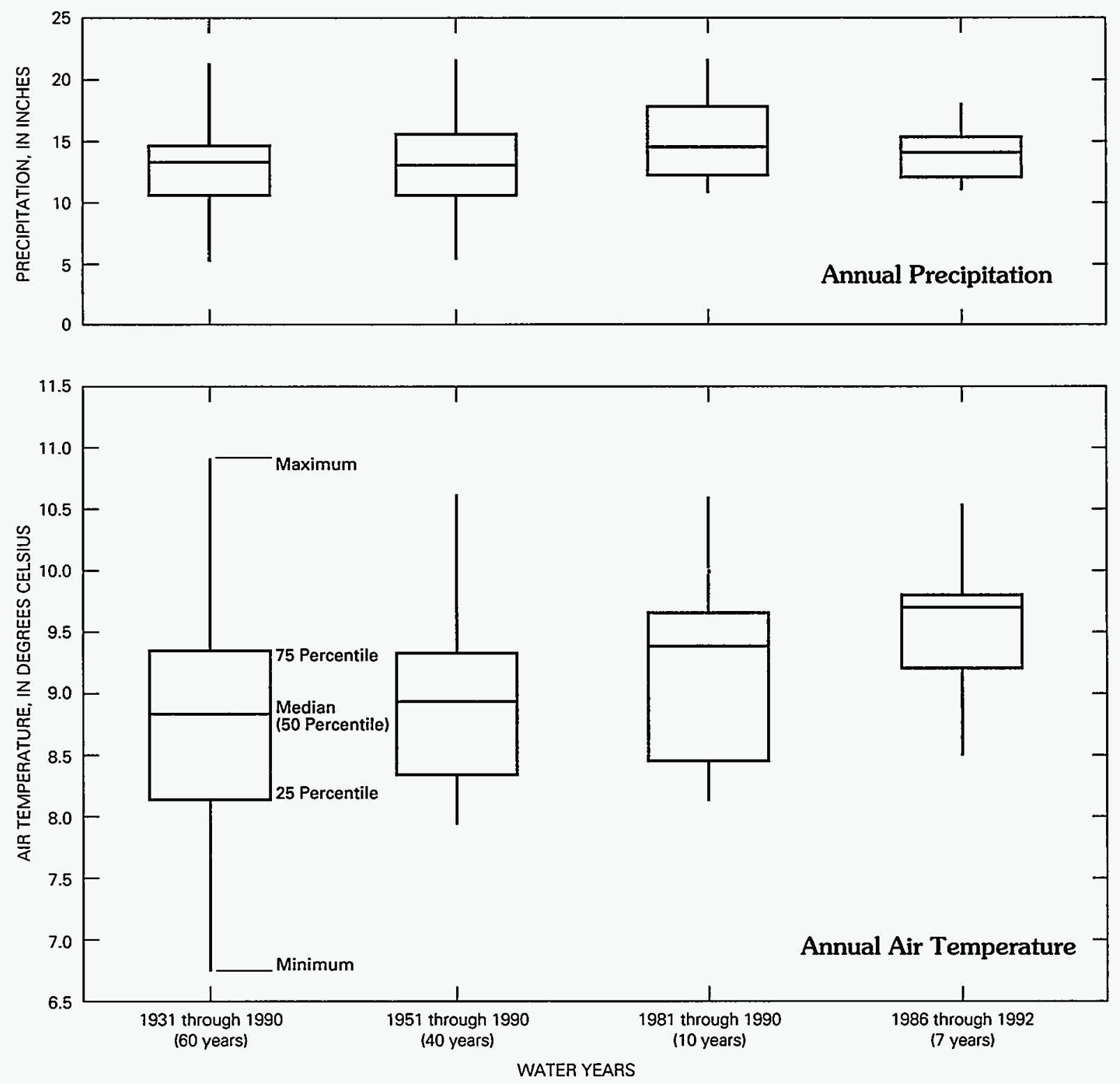

Figure 2. Annual air temperature and annual precipitation at Austin, Nevada. 


\section{REFERENCES}

Bird, R.E., and Hulstrum, R.L., 1981, Simplified clear sky model for direct and diffuse insolation on horizontal surface: Solar Energy Research Institute, TR-761, 47 p. (NNA.931124.0013)

Dempster, G.R., complier, 1990, National water information system user's manual, volume 2 , chapter 3 , Automated data processing system: U.S. Geological Survey OpenFile Report $90-116$, variously paginated. (NNA.931124.0014)

Dodge, R.A., 1990, Effects of mountain stream topography on the accuracy of small Parshall flumes: Bureau of Reclamation, Report R-90-03, 15 p. (NNA.931109.0002)

Rantz, S.E., 1982, Measurement and computation of streamflow, volume 2-Computation of discharge: U.S. Geo- logical Survey Water-Supply Paper 2175, p. 285-631. (NNA.931109.0003)

U.S. Bureau of Reclamation, 1984, Water measurement manual (2nd ed.): U.S. Bureau of Reclamation, $327 \mathrm{p}$. (NNA.931026.0002)

Voegelli, P.T., Sr., and Claassen, H.C., 1971, Radiochemical analyses of water from selected streams, wells, springs, and precipitation collected prior to reentry drilling, Project Rulison: U.S. Geological Survey Open-File Report USGS-474-101, 14 p. (NNA.931201.0115)

NOTE: Parenthesized numbers following each cited reference are for U.S. Department of Energy OCRWM Records Management purposes only and should not be used when ordering the publication. 


\section{RECORDS FROM 3-SPRINGS BASIN}




\section{SPRINGS BASIN}

\section{KAWICH PEAK NEAR WARM SPRINGS, NV}

LOCATION. -- Lat $37^{\circ} 57^{\prime} 19^{\prime}$, long $116^{\circ} 26^{\prime} 57^{\prime}$, Nye County, Hydrologic Unit 16060012, 1.4 mi west of the 3 Springs Creek surface-water equipment, $3.9 \mathrm{mi}$ west of Eden Ranch in the Reveille Valley, and $16.8 \mathrm{mi}$ south of Warm Springs at the intersection of US Highway 6 and Nevada Highway 375.

PERIOD OF RECORD. -- October 1988 to current year.

REMARKS. -- Values for the daily maximum and minimum air temperature were obtained from the forty minute average values. Estimated periods of daily record are considered poor.

PERIOD OF DAILY RECORD. --

AIR TEMPERATURE: October 1988 to current year.

SOIL TEMPERATURE: October 1988 to current year.

SOLAR RADIATION: November 1990 to current year.

RELATIVE HUMIDITY: October 1990 to current year.

TOTAL PRECIPITATION: October 1988 to current year.

EXTREMES FOR PERIOD OF RECORD. --

AIR TEMPERATURE: Maximum daily, $26.6^{\circ} \mathrm{C}$, July 4, 1991; minimum daily, -25.5 C, Feb. $6,1989$. See REMARKS for more information.

SOIL TEMPERATURE: Maximum daily mean, $20.0^{\circ} \mathrm{C}$, Aug 25,1991 ; minimum daily mean, $-6.1^{\circ} \mathrm{C}$,

Dec. $30,1990$.

SOLAR RADIATION: Maximum daily total, $799 \mathrm{cal} / \mathrm{cm}^{2}$, June 22-23, 1991; minimum daily total, $25 \mathrm{cal} / \mathrm{cm}^{2}$, Dec. 13, 1990.

RELATIVE HUMIDITY: Maximum daily mean, 100 pct, Mar. 4, 1991; minimum daily mean, 14.4 pct, July 29, 1992.

TOTAL PRECIPITATION: Maximum daily, 1.45 in, May 29, 1990; no precipitation, most days of record.

EXTREMES FOR CURRENT YEAR. --

AIR TEMPERATURE: Maximum daily, $25.4^{\circ} \mathrm{C}$, Aug. 18; minimum daily, $-14.9^{\circ} \mathrm{C}$, Nov. 30 . See REMARKS for more information.

SOIL TEMPERATURE: Maximum daily mean, $16.9^{\circ} \mathrm{C}$, Aug $19-20$; minimum daily mean, $-3.4^{\circ} \mathrm{C}$, Dec. 22. SOLAR RADIATION: Maximum daily total, $773 \mathrm{cal} / \mathrm{cm}^{2}$, May 20; minimum daily total, $31 \mathrm{cal} / \mathrm{cm}^{2}$,

Dec. 28 .

RELATIVE HUMIDITY: Maximum daily mean, 99.8 pct, Dec. 18; minimum daily mean, 14.4 pct, July 29. TOTAL PRECIPITATION: Maximum daily, 1.21 in, Jan. 5; no precipitation, most days of record. 


\section{SPRINGS BASIN}

KAWICH PEAK NEAR WARM SPRINGS, NV

DAILY MEAN AIR TEMPERATURE, WATER YEAR OCTOBER 1991 TO SEPTEMBER 1992 DEGREES CELSIUS

\begin{tabular}{|c|c|c|c|c|c|c|c|c|c|c|c|c|}
\hline DAY & OCT & NOV & DEC & JAN & FEB & MAR & APR & MAY & JUN & JUL & AUG & SEP \\
\hline $\begin{array}{l}1 \\
2 \\
3 \\
4 \\
5\end{array}$ & $\begin{array}{l}12.1 \\
12.9 \\
13.1 \\
11.7 \\
14.3\end{array}$ & $\begin{array}{l}2.0 \\
2.1 \\
3.7 \\
6.2 \\
7.9\end{array}$ & $\begin{array}{r}-8.5 \\
-1.1 \\
.1 \\
2.6 \\
2.0\end{array}$ & $\begin{array}{l}-2.3 \\
-2.9 \\
-4.4 \\
-5.1 \\
-4.9\end{array}$ & $\begin{array}{l}-2.6 \\
-3.9 \\
-5.5 \\
-3.7 \\
-2.5\end{array}$ & $\begin{array}{l}-1.8 \\
-3.1 \\
-1.7 \\
-1.2 \\
-1.4\end{array}$ & $\begin{array}{l}2.5 \\
5.1 \\
7.0 \\
5.8 \\
4.5\end{array}$ & $\begin{array}{r}5.3 \\
7.2 \\
9.8 \\
11.2 \\
9.7\end{array}$ & $\begin{array}{l}16.0 \\
15.7 \\
16.1 \\
17.3 \\
15.4\end{array}$ & $\begin{array}{r}6.5 \\
10.7 \\
14.1 \\
15.1 \\
15.4\end{array}$ & $\begin{array}{l}17.5 \\
19.8 \\
20.1 \\
17.5 \\
15.6\end{array}$ & $\begin{array}{r}11.4 \\
12.1 \\
10.9 \\
7.0 \\
11.0\end{array}$ \\
\hline $\begin{array}{r}6 \\
7 \\
8 \\
9 \\
10\end{array}$ & $\begin{array}{l}14.1 \\
13.3 \\
11.8 \\
11.0 \\
12.2\end{array}$ & $\begin{array}{l}8.4 \\
6.5 \\
6.9 \\
4.4 \\
1.9\end{array}$ & $\begin{array}{r}3.3 \\
-3.4 \\
-5.9 \\
-3.1 \\
-2.3\end{array}$ & $\begin{array}{r}-9.4 \\
-8.7 \\
-7.2 \\
-.4 \\
2.6\end{array}$ & $\begin{array}{l}-3.6 \\
-2.8 \\
-2.3 \\
-3.6 \\
-4.4\end{array}$ & $\begin{array}{r}-4.3 \\
-6.0 \\
-3.4 \\
-1.6 \\
.8\end{array}$ & $\begin{array}{l}4.6 \\
4.5 \\
6.8 \\
7.2 \\
6.9\end{array}$ & $\begin{array}{r}7.0 \\
9.2 \\
10.7 \\
7.3 \\
8.2\end{array}$ & $\begin{array}{l}10.6 \\
11.5 \\
11.1 \\
13.5 \\
13.2\end{array}$ & $\begin{array}{l}16.0 \\
14.3 \\
13.3 \\
15.6 \\
16.1\end{array}$ & $\begin{array}{l}14.0 \\
16.4 \\
16.9 \\
18.4 \\
18.8\end{array}$ & $\begin{array}{l}11.8 \\
12.4 \\
15.0 \\
14.6 \\
15.5\end{array}$ \\
\hline $\begin{array}{l}11 \\
12 \\
13 \\
14 \\
15\end{array}$ & $\begin{array}{l}12.9 \\
10.5 \\
12.2 \\
13.0 \\
14.3\end{array}$ & $\begin{array}{r}3.7 \\
5.8 \\
5.2 \\
-5.9 \\
-6.9\end{array}$ & $\begin{array}{r}-3.7 \\
-1.0 \\
-1.2 \\
1.9 \\
2.5\end{array}$ & $\begin{array}{r}-5.4 \\
-10.1 \\
-2.7 \\
-4.5 \\
-.6\end{array}$ & $\begin{array}{l}-3.9 \\
-3.5 \\
-3.4 \\
-5.2 \\
-6.5\end{array}$ & $\begin{array}{l}1.8 \\
2.8 \\
2.4 \\
1.3 \\
-.2\end{array}$ & $\begin{array}{l}6.8 \\
6.2 \\
6.0 \\
4.2 \\
3.8\end{array}$ & $\begin{array}{l}11.3 \\
11.2 \\
10.7 \\
11.3 \\
12.0\end{array}$ & $\begin{array}{r}12.4 \\
7.4 \\
4.2 \\
.6 \\
1.1\end{array}$ & $\begin{array}{r}12.2 \\
9.3 \\
10.5 \\
13.0 \\
13.4\end{array}$ & $\begin{array}{l}19.8 \\
19.2 \\
16.4 \\
17.3 \\
18.4\end{array}$ & $\begin{array}{l}14.0 \\
13.2 \\
12.3 \\
12.4 \\
12.2\end{array}$ \\
\hline $\begin{array}{l}16 \\
17 \\
18 \\
19 \\
20\end{array}$ & $\begin{array}{r}13.0 \\
12.6 \\
12.6 \\
9.4 \\
5.3\end{array}$ & $\begin{array}{r}.0 \\
-.4 \\
-5.9 \\
-1.5 \\
4.5\end{array}$ & $\begin{array}{r}.6 \\
-.7 \\
-2.3 \\
-9.0 \\
-7.2\end{array}$ & $\begin{array}{r}1.9 \\
-2.9 \\
-6.4 \\
-4.2 \\
-3.6\end{array}$ & $\begin{array}{r}-6.8 \\
-5.7 \\
-4.6 \\
-.5 \\
1.3\end{array}$ & $\begin{array}{r}-2.5 \\
-4.0 \\
-2.7 \\
-.5 \\
-.8\end{array}$ & $\begin{array}{r}5.2 \\
6.7 \\
.1 \\
1.4 \\
5.8\end{array}$ & $\begin{array}{r}12.1 \\
12.2 \\
12.0 \\
9.6 \\
7.7\end{array}$ & $\begin{array}{r}5.1 \\
9.7 \\
12.1 \\
12.7 \\
13.3\end{array}$ & $\begin{array}{l}15.5 \\
16.6 \\
17.3 \\
18.2 \\
17.6\end{array}$ & $\begin{array}{l}17.8 \\
19.1 \\
20.8 \\
20.8 \\
19.6\end{array}$ & $\begin{array}{r}12.9 \\
12.3 \\
6.8 \\
11.3 \\
13.8\end{array}$ \\
\hline $\begin{array}{l}21 \\
22 \\
23 \\
24 \\
25\end{array}$ & $\begin{array}{r}6.6 \\
5.9 \\
2.0 \\
-.8 \\
.9\end{array}$ & $\begin{array}{r}.1 \\
-4.9 \\
.9 \\
3.8 \\
3.7\end{array}$ & $\begin{array}{l}-2.9 \\
-2.9 \\
-2.6 \\
-2.6 \\
-1.1\end{array}$ & $\begin{array}{r}-2.1 \\
-.5 \\
1.9 \\
2.8 \\
3.1\end{array}$ & $\begin{array}{r}3.0 \\
.3 \\
-2.6 \\
1.3 \\
.7\end{array}$ & $\begin{array}{r}-1.9 \\
-3.1 \\
-2.7 \\
-.9 \\
.2\end{array}$ & $\begin{array}{r}4.3 \\
4.1 \\
4.6 \\
7.9 \\
10.2\end{array}$ & $\begin{array}{r}6.4 \\
7.4 \\
9.5 \\
13.0 \\
13.8\end{array}$ & $\begin{array}{l}14.8 \\
16.2 \\
14.9 \\
13.4 \\
10.8\end{array}$ & $\begin{array}{l}16.2 \\
15.3 \\
14.5 \\
14.9 \\
15.5\end{array}$ & $\begin{array}{l}17.0 \\
12.3 \\
10.4 \\
11.8 \\
13.3\end{array}$ & $\begin{array}{r}14.6 \\
15.9 \\
14.7 \\
13.5 \\
8.2\end{array}$ \\
\hline $\begin{array}{l}26 \\
27 \\
28 \\
29 \\
30 \\
31\end{array}$ & $\begin{array}{r}-1.1 \\
-7.9 \\
-7.9 \\
-6.1 \\
-9.8 \\
.7\end{array}$ & $\begin{array}{r}4.9 \\
-.6 \\
-8.3 \\
-10.7 \\
-12.9 \\
---\end{array}$ & $\begin{array}{l}-.9 \\
-2.2 \\
-6.1 \\
-6.6 \\
-5.7 \\
-4.7\end{array}$ & $\begin{array}{r}1.0 \\
.5 \\
-.9 \\
-.5 \\
2.0 \\
1.9\end{array}$ & $\begin{array}{l}2.6 \\
3.2 \\
2.7 \\
1.5 \\
--- \\
---\end{array}$ & $\begin{array}{r}.0 \\
.6 \\
.5 \\
.3 \\
-1.0 \\
.1\end{array}$ & $\begin{array}{r}12.1 \\
13.0 \\
13.2 \\
12.1 \\
9.4 \\
---\end{array}$ & $\begin{array}{l}13.9 \\
12.8 \\
10.2 \\
10.3 \\
10.7 \\
14.0\end{array}$ & $\begin{array}{r}13.4 \\
16.4 \\
15.9 \\
10.7 \\
9.5 \\
---\end{array}$ & $\begin{array}{l}17.3 \\
17.8 \\
19.5 \\
20.2 \\
19.6 \\
16.6\end{array}$ & $\begin{array}{l}13.4 \\
15.0 \\
15.9 \\
15.0 \\
11.0 \\
10.7\end{array}$ & $\begin{array}{r}10.1 \\
13.7 \\
14.2 \\
15.3 \\
14.4 \\
-.-\end{array}$ \\
\hline $\begin{array}{l}\text { MEAN } \\
\text { MAX } \\
\text { MIN } \\
\text { MED }\end{array}$ & $\begin{array}{r}7.3 \\
14.3 \\
-9.8 \\
11.7\end{array}$ & $\begin{array}{r}.8 \\
8.4 \\
-12.9 \\
2.0\end{array}$ & $\begin{array}{r}-2.4 \\
3.3 \\
-9.0 \\
-2.3\end{array}$ & $\begin{array}{r}-2.3 \\
3.1 \\
-10.1 \\
-2.3\end{array}$ & $\begin{array}{r}-2.1 \\
3.2 \\
-6.8 \\
-2.8\end{array}$ & $\begin{array}{r}-1.1 \\
2.8 \\
-6.0 \\
-1.0\end{array}$ & $\begin{array}{r}6.4 \\
13.2 \\
.1 \\
5.9\end{array}$ & $\begin{array}{r}10.2 \\
14.0 \\
5.3 \\
10.7\end{array}$ & $\begin{array}{r}11.8 \\
17.3 \\
.6 \\
13.0\end{array}$ & $\begin{array}{r}15.1 \\
20.2 \\
6.5 \\
15.5\end{array}$ & $\begin{array}{l}16.5 \\
20.8 \\
10.4 \\
17.0\end{array}$ & $\begin{array}{r}12.6 \\
15.9 \\
6.8 \\
12.6\end{array}$ \\
\hline
\end{tabular}

CAL YR 1991 MEAN 5.0 $\operatorname{MAX} 21.3$ MIN -12.9 MED 3.3 WTR YR 1992
MEAN 6.1 MAX 20.8 MIN -12.9 MED 6.5 
3 SPRINGS BASIN

KAWICH PEAK NEAR WARM SPRINGS, NV

DAILY MAXIMUM AIR TEMPERATURE, WATER YEAR OCTOBER 1991 TO SEPTEMBER 1992 DEGREES CELSIUS

\begin{tabular}{|c|c|c|c|c|c|c|c|c|c|c|c|c|}
\hline DAY & OCT & NOV & DEC & JAN & FEB & MAR & APR & MAY & JUN & JUL & AUG & SEP \\
\hline 1 & 17.9 & 5.2 & -1.8 & 3.1 & -.8 & -.6 & 6.5 & 10.9 & 21.7 & 12.2 & 23.3 & 16.6 \\
\hline 2 & 18.0 & 5.8 & 2.5 & 3.4 & -.9 & -2.3 & 10.0 & 12.5 & 21.2 & 16.6 & 24.0 & 16.9 \\
\hline 3 & 18.3 & 7.7 & 4.4 & -1.9 & -2.8 & .4 & 13.3 & 14.9 & 20.9 & 18.7 & 24.3 & 13.5 \\
\hline 4 & 17.6 & 9.8 & 8.1 & -1.4 & .2 & 3.1 & 10.3 & 15.0 & 22.1 & 20.2 & 21.7 & 13.0 \\
\hline 5 & 18.9 & 11.6 & 9.5 & -3.4 & -.5 & 2.2 & 8.7 & 14.8 & 20.0 & 19.9 & 20.7 & 15.7 \\
\hline 6 & 18.9 & 12.1 & 9.8 & -7.4 & -3.1 & -2.5 & 9.9 & 12.3 & 15.0 & 20.7 & 19.2 & 16.7 \\
\hline 7 & 17.4 & 10.5 & -1.2 & -2.7 & -.4 & -3.7 & 9.4 & 15.3 & 17.6 & 19.7 & 20.5 & 18.5 \\
\hline 8 & 16.8 & 10.2 & -2.1 & -2.0 & .6 & -.8 & 11.5 & 15.5 & 16.5 & 16.7 & 21.2 & 19.7 \\
\hline 9 & 15.8 & 8.4 & 2.5 & 4.9 & -2.7 & 2.1 & 11.5 & 12.8 & 17.5 & $21 \cdot 3$ & 23.7 & 21.3 \\
\hline 10 & 17.5 & 5.1 & 2.4 & 7.3 & -3.7 & 6.1 & 11.9 & 14.2 & 16.8 & 20.9 & 23.4 & 20.4 \\
\hline 11 & 17.3 & 8.1 & .0 & 2.8 & -1.4 & 7.5 & 10.7 & 16.6 & 16.6 & 16.5 & 24.5 & 17.6 \\
\hline 12 & 14.2 & 11.0 & 2.9 & -7.0 & -2.3 & 8.8 & 9.9 & 16.3 & 10.8 & 14.1 & 23.8 & 16.9 \\
\hline 13 & 16.5 & 9.0 & 2.6 & 1.2 & .7 & 5.8 & 10.1 & 15.6 & 9.6 & 15.0 & 23.7 & 17.8 \\
\hline 14 & 17.3 & -2.0 & 5.8 & -1.2 & -.9 & 4.9 & 8.3 & 16.1 & 2.7 & 18.2 & 23.1 & 16.7 \\
\hline 15 & 19.4 & -4.1 & 6.7 & 5.3 & -5.1 & 3.8 & 8.1 & 17.1 & 6.0 & 17.9 & 23.7 & 16.4 \\
\hline 16 & 17.2 & 4.5 & 7.1 & 7.4 & -1.0 & 3.7 & 9.6 & 17.9 & 11.0 & 20.1 & 23.4 & 17.6 \\
\hline 17 & 16.7 & 3.0 & 1.7 & -.2 & 1.4 & -.6 & 11.3 & 16.5 & 16.5 & 23.7 & 24.4 & 17.5 \\
\hline 18 & 16.3 & -3.6 & -.8 & -4.4 & -.2 & 2.3 & 4.1 & 16.6 & 17.2 & 22.9 & 25.4 & 10.7 \\
\hline 19 & 14.7 & 4.0 & -4.9 & -1.4 & 3.5 & 4.1 & 8.1 & 12.9 & 18.7 & 23.0 & 25.1 & 17.1 \\
\hline 20 & 9.6 & 8.9 & -2.1 & 1.3 & 6.6 & 3.0 & 11.9 & 12.6 & 19.2 & 22.5 & 23.6 & 19.0 \\
\hline 21 & 11.5 & 3.2 & 3.0 & 1.4 & 7.8 & 1.2 & 9.4 & 13.0 & 20.4 & 22.2 & 21.1 & 20.8 \\
\hline 22 & 9.4 & -.3 & 1.8 & 2.8 & 2.9 & -2.1 & 8.5 & 13.3 & 21.0 & 19.3 & 17.3 & 20.2 \\
\hline 23 & 6.1 & 7.4 & .0 & 5.4 & 1.7 & 1.9 & 10.6 & 15.4 & 19.2 & 19.0 & 16.3 & 18.4 \\
\hline 24 & 2.5 & 7.7 & 1.6 & 8.8 & 6.7 & $2 \cdot 5$ & 14.0 & 17.9 & 17.5 & 19.7 & 17.7 & 18.7 \\
\hline 25 & 5.1 & 6.6 & 2.7 & 7.3 & 4.2 & 3.3 & 16.3 & 19.5 & 14.1 & 20.8 & 18.1 & 13.3 \\
\hline 26 & 2.6 & 8.7 & 3.5 & 5.2 & 7.5 & 3.5 & 16.9 & 17.9 & 18.3 & 23.0 & 19.1 & 17.3 \\
\hline 27 & -5.1 & 4.7 & 1.0 & 6.9 & 9.6 & 5.4 & 18.3 & 17.3 & 22.2 & 21.3 & 20.7 & 19.5 \\
\hline 28 & -4.3 & -4.9 & -5.1 & 1.7 & 8.4 & 5.8 & 19.0 & 13.1 & 19.7 & 24.3 & 19.8 & 20.4 \\
\hline 29 & -3.5 & -5.1 & -5.8 & 2.9 & 4.9 & 4.6 & 16.4 & 15.3 & 14.7 & 24.7 & 19.0 & 19.4 \\
\hline 30 & -5.7 & -10.1 & -2.1 & 7.6 & --- & 1.4 & 14.0 & 15.1 & 14.4 & 24.4 & 15.5 & 18.2 \\
\hline 31 & 4.9 & --- & -.4 & 5.1 & --- & 3.7 & $-\infty$ & 19.3 & --- & 22.1 & 16.5 & -- \\
\hline MAX & 19.4 & 12.1 & 9.8 & 8.8 & 9.6 & 8.8 & 19.0 & 19.5 & 22.2 & 24.7 & 25.4 & 21.3 \\
\hline CAL YR & 1991 & MAX 26.6 & & & & & & & & & & \\
\hline WTR YR & 1992 & $\operatorname{MAX} 25.4$ & & & & & & & & & & \\
\hline
\end{tabular}




\section{SPRINGS BASIN}

KAWICH PEAK NEAR WARM SPRINGS, NV

DAILY MINIMUM AIR TEMPERATURE, WATER YEAR·OCTOBER 1991 TO SEPTEMBER 1992 DEGREES CELSIUS

\begin{tabular}{|c|c|c|c|c|c|c|c|c|c|c|c|c|}
\hline DAY & OCT & NOV & DEC & JAN & FEB & MAR & $A P R$ & MAY & JUN & JUL & AUG & SEP \\
\hline 1 & 6.2 & .0 & -14.3 & -7.2 & -5.5 & -2.7 & -.7 & .1 & 7.8 & 1.0 & 11.0 & 5.7 \\
\hline 2 & 8.3 & -.5 & -4.6 & -7.7 & -5.7 & $-\dot{4} .6$ & .3 & 1.9 & 9.1 & 2.6 & 15.3 & 7.1 \\
\hline 3 & 7.5 & 1.1 & -3.4 & -6.5 & -8.0 & -3.2 & .7 & 2.9 & 9.3 & 10.0 & 14.3 & 5.8 \\
\hline 4 & 6.9 & 2.9 & -2.4 & -6.6 & -6.7 & -4.6 & 2.2 & 7.3 & 10.7 & 10.6 & 14.3 & 1.9 \\
\hline 5 & 10.6 & 5.0 & -2.5 & -7.1 & -4.2 & -5.5 & 1.0 & 5.4 & 9.7 & 11.1 & 9.9 & 4.1 \\
\hline 6 & 8.7 & 5.5 & -1.4 & -10.9 & -4.0 & -9.3 & -.6 & 4.7 & 5.6 & 11.7 & 9.1 & 8.4 \\
\hline 7 & 10.7 & 3.8 & -6.3 & -10.9 & -4.4 & -10.7 & -1.7 & 1.5 & 4.4 & 8.3 & 12.4 & 4.9 \\
\hline 8 & 6.9 & 4.5 & -7.7 & -11.0 & -3.8 & -5.1 & 1.7 & 7.7 & 7.3 & 9.9 & 12.9 & 10.2 \\
\hline 9 & 6.8 & 1.1 & -7.3 & -3.5 & -4.8 & -4.7 & 3.6 & 1.5 & 9.2 & 10.3 & 12.8 & 7.8 \\
\hline 10 & 8.6 & .0 & -5.6 & -1.2 & -5.3 & -4.9 & 3.3 & 1.1 & 9.6 & 11.0 & 13.9 & 8.9 \\
\hline 11 & 9.8 & -.4 & -6.5 & -12.9 & -5.5 & -1.9 & 3.6 & 6.5 & 7.7 & 6.3 & 13.8 & 11.1 \\
\hline 12 & 7.4 & .1 & -4.6 & -13.8 & -4.3 & -3.2 & 3.6 & 6.2 & 1.0 & 6.2 & 14.2 & 9.5 \\
\hline 13 & 7.1 & -1.8 & -4.7 & -6.5 & -5.5 & -.4 & 2.2 & 6.3 & -2.0 & 6.9 & 10.9 & 5.1 \\
\hline 14 & 8.9 & -9.5 & -2.7 & -8.1 & -9.3 & -1.1 & -.4 & 6.7 & -2.2 & 6.2 & 10.9 & 9.2 \\
\hline 15 & 8.0 & -9.4 & -2.4 & -5.4 & -8.6 & -3.2 & .1 & 7.0 & -2.3 & 9.2 & 12.6 & 8.0 \\
\hline 16 & 9.6 & -6.8 & -2.9 & -3.6 & -10.7 & -5.6 & -1.1 & 5.6 & -1.3 & 8.9 & 12.0 & 9.5 \\
\hline 17 & 8.3 & -5.1 & -2.3 & -6.8 & -11.0 & -6.6 & 1.7 & 7.9 & 2.0 & 11.0 & 13.3 & 7.1 \\
\hline 18 & 8.5 & -8.2 & -4.5 & -8.2 & -8.7 & -6.7 & -3.9 & 7.6 & 7.6 & 10.0 & 16.4 & 3.8 \\
\hline 19 & 5.0 & -8.9 & -11.1 & -7.6 & -5.1 & -5.8 & -4.5 & 5.4 & 4.4 & 12.5 & 15.6 & 5.3 \\
\hline 20 & 1.6 & .7 & -11.2 & -7.6 & -2.4 & -2.8 & -.5 & 2.3 & 8.1 & 11.1 & 14.1 & 8.9 \\
\hline 21 & .7 & -7.4 & -6.3 & -5.5 & .0 & -4.0 & -.3 & 3.2 & 7.8 & 8.0 & 12.1 & 10.2 \\
\hline 22 & 2.9 & -9.4 & -7.1 & -2.7 & -5.1 & -5.4 & .1 & 3.3 & 10.8 & 12.0 & 7.3 & 11.9 \\
\hline 23 & -2.8 & -5.8 & -5.1 & -2.4 & -6.8 & -5.2 & -1.3 & 1.8 & 10.1 & 10.1 & 2.6 & 11.9 \\
\hline 24 & -4.7 & -.3 & -6.8 & -2.4 & -3.4 & -5.8 & 1.0 & 6.5 & 9.7 & 10.4 & 4.2 & 7.5 \\
\hline 25 & -2.1 & -.6 & -4.0 & -.6 & -1.6 & -2.2 & 3.4 & 8.5 & 5.3 & 10.3 & 8.6 & 4.0 \\
\hline 26 & -6.2 & 2.8 & -3.9 & -2.4 & -2.0 & -2.9 & 5.5 & 10.7 & 6.3 & 12.6 & 7.1 & 4.3 \\
\hline 27 & -11.4 & -12.6 & -4.8 & -4.9 & -3.1 & -3.1 & 8.5 & 7.6 & 8.3 & 13.9 & 7.9 & 9.6 \\
\hline 28 & -11.8 & -12.5 & -6.7 & -3.3 & -2.8 & -2.9 & 5.7 & 6.0 & 10.5 & 13.5 & 12.5 & 8.0 \\
\hline 29 & -10.6 & -14.2 & -7.1 & -3.4 & -1.0 & -3.7 & 8.5 & 5.3 & 6.7 & 15.9 & 10.6 & 11.5 \\
\hline 30 & -12.7 & -14.9 & -8.9 & -1.8 & -- & -2.3 & 3.9 & 6.4 & 4.5 & 15.2 & 7.2 & 11.0 \\
\hline 31 & -4.8 & -- & -10.5 & -1.4 & --- & -2.1 & --- & 9.0 & -- & 10.7 & 5.8 & --- \\
\hline MIN & -12.7 & -14.9 & -14.3 & -13.8 & -11.0 & -10.7 & -4.5 & .1 & -2.3 & 1.0 & 2.6 & 1.9 \\
\hline
\end{tabular}

CAL YR 1991 MIN -14.9

WTR YR 1992 MIN -14.9 


\section{SPRINGS BASIN}

KAWICH PEAK NEAR WARM SPRINGS, NV

DAILY MEAN SOIL TEMPERATURE, WATER YEAR OCTOBER 1991 TO SEPTEMBER 1992 DEGREES CELSIUS

\begin{tabular}{|c|c|c|c|c|c|c|c|c|c|c|c|c|}
\hline DAY & OCT & NOV & DEC & JAN & FEB & MAR & APR & MAY & JUN & JUL & AUG & SEP \\
\hline 1 & 8.5 & 2.9 & -.7 & -2.7 & -1.6 & -.3 & .0 & 7.8 & 11.2 & 12.8 & 15.6 & 12.3 \\
\hline 2 & 8.9 & 2.7 & -.8 & -3.1 & -1.7 & -.2 & .0 & 7.8 & 12.5 & 12.4 & 16.1 & 12.3 \\
\hline 3 & 8.8 & 2.5 & -.7 & -3.2 & -1.8 & -.2 & .1 & 8.0 & 12.8 & 12.9 & 16.5 & 12.3 \\
\hline 4 & 8.9 & 2.3 & -.6 & -2.8 & -2.0 & -.2 & .1 & 8.4 & 13.3 & 13.6 & 16.7 & 11.4 \\
\hline 5 & 9.1 & 3.0 & -.8 & -2.6 & -2.1 & -.2 & .1 & 8.5 & 13.6 & 13.4 & 16.5 & 10.8 \\
\hline 6 & 9.3 & 4.1 & -1.0 & -2.3 & -1.9 & -.1 & .1 & 7.9 & 12.6 & 13.6 & 15.8 & 11.7 \\
\hline 7 & 9.8 & 4.7 & -.6 & -2.2 & -1.8 & -.1 & .1 & 8.4 & 11.7 & 14.1 & 15.5 & 11.2 \\
\hline 8 & 9.8 & 4.5 & -.8 & -2.1 & -1.6 & -.1 & .1 & 8.9 & 12.1 & 14.3 & 15.7 & 12.0 \\
\hline 9 & 9.4 & 4.8 & -1.0 & -2.1 & -1.4 & -.1 & .2 & 8.8 & 11.8 & 13.7 & 16.0 & 12.0 \\
\hline 10 & 9.4 & 4.9 & -1.4 & -2.0 & -1.2 & -.1 & .2 & 8.7 & 12.6 & 14.3 & 16.3 & 12.4 \\
\hline 11 & 9.3 & 4.1 & -1.5 & -1.9 & -1.1 & -.1 & .2 & 9.6 & 12.8 & 14.1 & 16.0 & 13.0 \\
\hline 12 & 9.2 & 3.3 & -1.8 & -1.9 & -1.1 & -.1 & .2 & 10.4 & 12.4 & 11.2 & 16.1 & 12.9 \\
\hline 13 & 8.8 & 3.4 & -1.7 & -2.0 & -1.0 & -.1 & .2 & 10.6 & 10.6 & 10.9 & 15.5 & 12.0 \\
\hline 14 & 9.4 & 2.7 & -2.1 & -2.0 & -1.0 & -.1 & .2 & 10.6 & 9.7 & 10.8 & 15.0 & 12.2 \\
\hline 15 & 9.6 & 1.5 & -1.7 & -1.9 & -.9 & -.1 & .2 & 10.8 & 8.3 & 11.7 & 15.4 & 12.1 \\
\hline 16 & 9.8 & .8 & -2.3 & -1.9 & -.9 & .0 & .2 & 11.1 & 8.3 & 12.2 & 15.5 & 12.3 \\
\hline 17 & 9.9 & .9 & -2.1 & -1.7 & -.9 & .0 & .2 & 11.3 & 9.3 & 13.6 & 16.0 & 12.3 \\
\hline 18 & 9.8 & 1.0 & -1.4 & -1.7 & -.9 & .0 & .2 & 11.4 & 10.7 & 14.1 & 16.6 & 10.3 \\
\hline 19 & 9.4 & .5 & -1.4 & -1.8 & -.8 & .0 & .3 & 10.9 & 11.2 & 15.0 & 16.9 & 9.8 \\
\hline 20 & 8.9 & .6 & -2.2 & -1.9 & -.8 & .0 & .5 & 10.4 & 12.0 & 15.1 & 16.9 & 10.3 \\
\hline 21 & 7.6 & 1.1 & -2.9 & -2.0 & -.8 & .0 & .8 & 10.2 & 11.9 & 15.1 & 16.5 & 11.0 \\
\hline 22 & 7.8 & .9 & -3.4 & -2.0 & -.7 & .0 & 1.3 & 10.1 & 12.6 & 15.3 & 15.9 & 11.6 \\
\hline 23 & 7.5 & .4 & -3.1 & -1.9 & -.6 & .0 & 2.4 & 10.2 & 12.7 & 15.2 & 14.9 & 12.2 \\
\hline 24 & 5.7 & .5 & -3.1 & -1.8 & -.5 & .0 & 3.5 & 10.8 & 12.5 & 15.1 & 13.8 & 12.2 \\
\hline 25 & 5.2 & .9 & -3.3 & -1.6 & -.5 & .0 & 4.8 & 11.4 & 12.1 & 15.5 & 14.2 & 11.5 \\
\hline 26 & 5.0 & 1.3 & -3.2 & -1.6 & -.4 & .0 & 6.1 & 11.3 & 12.8 & 15.4 & 14.1 & 10.4 \\
\hline 27 & 4.5 & 1.6 & -2.7 & -1.7 & -.4 & .0 & 7.3 & 11.6 & 13.5 & 14.8 & 13.6 & 10.4 \\
\hline 28 & 4.0 & 1.0 & -2.7 & -1.7 & -.4 & .0 & 7.9 & 11.3 & 14.2 & 15.2 & 13.9 & 10.3 \\
\hline 29 & 3.6 & .2 & -2.8 & -1.7 & -.3 & .0 & 8.2 & 10.8 & 13.9 & 16.2 & 14.4 & 10.6 \\
\hline 30 & 3.4 & -.1 & -2.6 & -1.7 & --- & .0 & 8.3 & 10.3 & 13.4 & 16.6 & 14.1 & 11.0 \\
\hline 31 & 3.0 & --- & -2.6 & -1.7 & --- & .0 & --- & 10.3 & --- & 16.3 & 12.9 & --- \\
\hline MEAN & 7.8 & 2.1 & -1.9 & -2.0 & -1.1 & -.1 & 1.8 & 10.0 & 12.0 & 14.0 & 15.4 & 11.6 \\
\hline MAX & 9.9 & 4.9 & -.6 & -1.6 & -.3 & .0 & 8.3 & 11.6 & 14.2 & 16.6 & 16.9 & 13.0 \\
\hline MIN & 3.0 & -.1 & -3.4 & -3.2 & -2.1 & -.3 & .0 & 7.8 & 8.3 & 10.8 & 12.9 & 9.8 \\
\hline MED & 8.9 & 1.5 & -1.8 & -1.9 & -.9 & .0 & .2 & 10.3 & 12.4 & 14.1 & 15.7 & 11.9 \\
\hline
\end{tabular}

CAL YR 1991 MEAN 5.5 MAX 20.0 MIN -5.9 MED 2.7

WTR YR 1992 MEAN 5.8 MAX 16.9 MIN -3.4 MED 5.1 
3 SPRINGS BASIN

KAWICH PEAK NEAR WARM SPRINGS, NV

DAILY TOTAL SOLAR RADIATION, INCIDENTAL, WATER YEAR OCTOBER 1991 TO SEPTEMBER 1992 CALORIES PER SQUARE CENTIMETER

\begin{tabular}{|c|c|c|c|c|c|c|c|c|c|c|c|c|}
\hline DAY & OCT & NOV & DEC & JAN & FEB & MAR & APR & MAY & JUN & JUL & AUG & SEP \\
\hline 1 & 515 & 343 & 244 & 260 & 176 & 172 & 512 & 591 & 654 & 592 & 619 & 412 \\
\hline 2 & 510 & 354 & 271 & 218 & 308 & 69 & 603 & 619 & 588 & 754 & 646 & 407 \\
\hline 3 & 502 & 331 & 278 & 62 & 350 & 180 & 622 & 650 & 753 & 590 & 688 & 289 \\
\hline 4 & 503 & 351 & 279 & 231 & 298 & 416 & 501 & 560 & 746 & 736 & 565 & 528 \\
\hline 5 & 489 & 352 & 281 & 47 & 142 & 407 & 628 & 525 & 769 & 465 & 551 & 580 \\
\hline 6 & 469 & 355 & 267 & 122 & 38 & 160 & 602 & 381 & 417 & 578 & 467 & 484 \\
\hline 7 & 431 & 353 & 75 & 211 & 84 & 165 & 593 & 687 & 729 & 725 & 659 & 595 \\
\hline 8 & 412 & 274 & 183 & 280 & 199 & 239 & 629 & 430 & 423 & 525 & 696 & 580 \\
\hline 9 & 377 & 271 & 254 & 276 & 133 & 384 & 552 & 731 & 736 & 570 & 677 & 577 \\
\hline 10 & 462 & 226 & 249 & 272 & 75 & 505 & 493 & 731 & 769 & 572 & 532 & 555 \\
\hline 11 & 354 & 342 & 254 & 241 & 143 & 508 & 537 & 730 & 769 & 336 & 492 & 417 \\
\hline 12 & 216 & 327 & 256 & 278 & 77 & 517 & 530 & 654 & 705 & 376 & 405 & 499 \\
\hline 13 & 451 & 273 & 256 & 254 & 181 & 487 & 640 & 678 & 662 & 302 & 356 & 576 \\
\hline 14 & 457 & 127 & 236 & 281 & 171 & 380 & 448 & 721 & 477 & 463 & 614 & 576 \\
\hline 15 & 455 & 116 & 194 & 286 & 60 & 514 & 292 & 694 & 317 & 469 & 602 & 555 \\
\hline 16 & 449 & 324 & 253 & 282 & 364 & 354 & 583 & 710 & 770 & 521 & 592 & 496 \\
\hline 17 & 439 & 284 & 114 & 189 & 367 & 445 & 455 & 754 & 743 & 618 & 648 & 497 \\
\hline 18 & 435 & 160 & 154 & 287 & 284 & 497 & 685 & 732 & 771 & 673 & 665 & 336 \\
\hline 19 & 427 & 308 & 182 & 305 & 361 & 490 & 688 & 526 & 770 & 736 & 653 & 536 \\
\hline 20 & 427 & 290 & 256 & 306 & 367 & 317 & 648 & 773 & 750 & 742 & 657 & 537 \\
\hline 21 & 422 & 305 & 254 & 294 & 304 & 297 & 362 & 471 & 731 & 741 & 658 & 537 \\
\hline 22 & 346 & 307 & 239 & 316 & 413 & 151 & 647 & 750 & 664 & 742 & 593 & 525 \\
\hline 23 & 391 & 299 & 183 & 314 & 440 & 485 & 652 & 666 & 406 & 744 & 648 & 419 \\
\hline 24 & 267 & 230 & 246 & 318 & 431 & 344 & 619 & 674 & 618 & 717 & 631 & 527 \\
\hline 25 & 354 & 251 & 251 & 209 & 431 & 356 & 644 & 529 & 512 & 725 & $=631$ & 524 \\
\hline 26 & 184 & 255 & 254 & 212 & 451 & 321 & 640 & 522 & 713 & 576 & 644 & 521 \\
\hline 27 & 166 & 259 & 243 & 325 & 457 & 377 & 701 & 707 & 764 & 362 & 646 & 512 \\
\hline 28 & 394 & 270 & 31 & 105 & 457 & 252 & 688 & 340 & 731 & 734 & 494 & 507 \\
\hline 29 & 144 & 157 & 70 & 331 & 398 & 379 & 611 & 534 & 663 & 730 & 540 & 410 \\
\hline 30 & 373 & 269 & 104 & 326 & --- & 217 & 692 & 397 & 699 & 717 & 619 & 420 \\
\hline 31 & 377 & --- & 252 & 344 & $\cdots$ & 228 & -- & 582 & -- & 390 & 436 & --- \\
\hline TOTAL & 12198 & 8363 & 6663 & 7782 & 7960 & 10613 & 17497 & 19049 & 19819 & 18521 & 18324 & 14934 \\
\hline CAL YR & 1991 & & & & & & & & & & & \\
\hline WTR YR & 1992 & TOTAL & 723 & & & & & & & & & \\
\hline
\end{tabular}


3 SPRINGS BASIN

KAWICH PEAK NEAR WARM SPRINGS, NV

DAILY MEAN RELATIVE HUMIDITY, WATER YEAR OCTOBER 1991 TO SEPTEMBER 1992 PERCENT

\begin{tabular}{|c|c|c|c|c|c|c|c|c|c|c|c|c|}
\hline DAY & OCT & NOV & DEC & JAN & FEB & MAR & APR & MAY & JUN & JUL & AUG & SEP \\
\hline 1 & 38 & 48 & 55 & 37 & 53 & 70 & 51 & 53 & 23 & 61 & 44 & 36 \\
\hline 2 & 26 & 62 & 43 & 22 & 68 & 97 & 27 & 35 & 28 & 39 & 29 & 30 \\
\hline 3 & 21 & 57 & 36 & 67 & 48 & 98 & 33 & 22 & 28 & 21 & 21 & 29 \\
\hline 4 & 18 & 54 & 25 & 95 & 72 & 89 & 52 & 22 & 18 & 19 & 31 & 65 \\
\hline 5 & 18 & 56 & 21 & 98 & 86 & 85 & 29 & 48 & 16 & 17 & 50 & 31 \\
\hline 6 & 21 & 58 & 20 & 95 & 98 & 97 & 34 & 79 & 41 & 22 & 58 & 21 \\
\hline 7 & 22 & 67 & 54 & 96 & 100 & 96 & 55 & 54 & 34 & 26 & 28 & 19 \\
\hline 8 & 28 & 64 & 97 & 81 & 99 & 92 & 40 & 45 & 43 & 57 & 18 & 18 \\
\hline 9 & 26 & 79 & 94 & 28 & 99 & 72 & 37 & 30 & 32 & 44 & 17 & 17 \\
\hline 10 & 22 & 90 & 70 & 25 & 98 & 52 & 35 & 21 & 22 & 37 & 19 & 17 \\
\hline 11 & 21 & 32 & 51 & 49 & 98 & 39 & 32 & 29 & 28 & 71 & 23 & 19 \\
\hline 12 & 35 & 21 & 42 & 59 & 98 & 41 & 23 & 37 & 26 & 86 & 34 & 19 \\
\hline 13 & 25 & 30 & 23 & 29 & 98 & 50 & 34 & 29 & 26 & 82 & 52 & 18 \\
\hline 14 & 19 & 74 & 21 & 66 & 97 & 56 & 65 & 19 & 53 & 56 & 50 & 18 \\
\hline 15 & 18 & 90 & 20 & 30 & 97 & 55 & 63 & 25 & 64 & 52 & 37 & 24 \\
\hline 16 & 19 & 52 & 22 & 26 & 96 & 83 & 56 & 22 & 57 & 43 & 36 & 39 \\
\hline 17 & 20 & 54 & 82 & 50 & 96 & 88 & 48 & 19 & 39 & 38 & 31 & 42 \\
\hline 18 & 22 & 78 & 100 & 55 & 97 & 56 & 39 & 21 & 21 & 33 & 17 & 86 \\
\hline 19 & 31 & 40 & 92 & 27 & 89 & 53 & 32 & 23 & 24 & 22 & 15 & 53 \\
\hline 20 & 46 & 35 & 53 & 41 & 75 & 57 & 26 & 25 & 23 & 17 & 15 & 34 \\
\hline 21 & 40 & 63 & 67 & 34 & 48 & 94 & 55 & 52 & 16 & 17 & 17 & 33 \\
\hline 22 & 68 & 40 & 67 & 21 & 66 & 97 & 27 & 53 & 17 & 19 & 26 & 39 \\
\hline 23 & 66 & 21 & 72 & 21 & 42 & 96 & 27 & 41 & 28 & 18 & 18 & 50 \\
\hline 24 & 59 & 34 & 57 & 21 & 31 & 93 & 25 & 34 & 32 & 20 & 18 & 33 \\
\hline 25 & 58 & 47 & 40 & 20 & 66 & 77 & 24 & 36 & 42 & 40 & 19 & 34 \\
\hline 26 & 88 & 26 & 35 & 22 & 34 & 79 & 21 & 31 & 41 & 20 & 17 & 26 \\
\hline 27 & 92 & 48 & 49 & 29 & 41 & 77 & 19 & 24 & 29 & 19 & 16 & 20 \\
\hline 28 & 61 & 65 & 95 & 42 & 39 & 83 & 17 & 43 & 24 & 15 & 18 & 18 \\
\hline 29 & 81 & 67 & 97 & 44 & 43 & 89 & 18 & 67 & 46 & 14 & 20 & 23 \\
\hline 30 & 69 & 63 & 97 & 21 & --- & 97 & 31 & 57 & 39 & 18 & 31 & 31 \\
\hline 31 & 39 & --- & 64 & 25 & $-\cdots$ & 94 & -- & 35 & --- & 40 & 37 & --- \\
\hline MEAN & 39 & 54 & 57 & 44 & 75 & 77 & 36 & 36 & 32 & 35 & 28 & 31 \\
\hline MAX & 92 & 90 & 100 & 98 & 100 & 98 & 65 & 79 & 64 & 86 & 58 & 86 \\
\hline MIN & 18 & 21 & 20 & 20 & 31 & 39 & 17 & 19 & 16 & 14 & 15 & 17 \\
\hline MED & 28 & 55 & 54 & 34 & 86 & 83 & 33 & 34 & 28 & 26 & 23 & 30 \\
\hline
\end{tabular}

CAL YR 1991 MEAN 46 MAX 100 MIN 15 MED 41

WTR YR 1992 MEAN 45 MAX 100 MIN 14 MED 39 
3 SPRINGS BASIN

KAWICH PEAK NEAR WARM SPRINGS, NV

DAILY TOTAL PRECIPITATION, WATER YEAR OCTOBER 1991 TO SEPTEMBER 1992

INCHES

\begin{tabular}{|c|c|c|c|c|c|c|c|c|c|c|c|c|}
\hline DAY & OCT & NOV & DEC & JAN & FEB & MAR & APR & MAY & JUN & JUL & AUG & SEP \\
\hline 1 & .00 & .00 & .00 & .02 & .00 & .03 & .00 & .00 & .00 & .00 & .00 & .00 \\
\hline 2 & .00 & .00 & .02 & .00 & .00 & .40 & .00 & .00 & .00 & .00 & .00 & .00 \\
\hline 3 & .00 & .00 & .00 & .15 & .00 & .25 & .02 & .00 & .00 & .00 & .00 & .00 \\
\hline 4 & .00 & .00 & .00 & .04 & .00 & .00 & .11 & .00 & .00 & .00 & .00 & .20 \\
\hline 5 & .00 & .00 & .00 & 1.21 & .00 & .10 & .00 & .10 & .00 & .00 & .10 & .00 \\
\hline 6 & .00 & .00 & .00 & .32 & .67 & .02 & .00 & .05 & .05 & .00 & .00 & .00 \\
\hline 7 & .00 & .00 & .17 & .00 & .15 & .23 & .00 & .02 & .02 & .00 & .00 & .00 \\
\hline 8 & .00 & .00 & .00 & .00 & .10 & .05 & .00 & .00 & .05 & .00 & .00 & .00 \\
\hline 9 & .00 & .00 & .00 & .00 & .10 & .00 & .00 & .00 & .00 & .00 & .00 & .00 \\
\hline 10 & .00 & .00 & .00 & .00 & .95 & .00 & .00 & .00 & .00 & .00 & .00 & .00 \\
\hline 11 & .00 & .00 & .00 & .00 & .50 & .00 & .00 & .00 & .00 & .97 & .00 & .00 \\
\hline 12 & .00 & .00 & .00 & .00 & .90 & .00 & .00 & .00 & .00 & .10 & .02 & .00 \\
\hline 13 & .00 & .00 & .00 & .00 & .15 & .00 & .00 & .00 & .00 & .07 & .18 & .00 \\
\hline 14 & .00 & .00 & .00 & .00 & .03 & .00 & .00 & .00 & .00 & .02 & .02 & .00 \\
\hline 15 & .00 & .00 & .00 & .00 & .32 & .00 & .05 & .00 & .02 & .00 & .00 & .00 \\
\hline 16 & .00 & .00 & .00 & .00 & .00 & .20 & .00 & .00 & .04 & .00 & .00 & .00 \\
\hline 17 & .00 & .02 & .00 & .00 & .00 & .05 & .00 & .00 & .00 & .01 & .00 & .40 \\
\hline 18 & .00 & .02 & .18 & .00 & .00 & .00 & .00 & .00 & .00 & .00 & .00 & .50 \\
\hline 19 & .00 & .00 & .03 & .00 & .00 & .00 & .00 & .00 & .00 & .00 & .00 & .00 \\
\hline 20 & .00 & .00 & .00 & .00 & .00 & .00 & .00 & .00 & .00 & .00 & .00 & .00 \\
\hline 21 & .00 & .00 & .00 & .00 & .04 & .25 & .12 & .00 & .00 & .00 & .00 & .00 \\
\hline 22 & .00 & .00 & .00 & .00 & .00 & 1.00 & .00 & .00 & .00 & .00 & .00 & .00 \\
\hline 23 & .00 & .00 & .00 & .00 & .00 & .06 & .00 & .00 & .00 & .00 & .00 & .00 \\
\hline 24 & .00 & .00 & .00 & .00 & .00 & .00 & .00 & .00 & .00 & .00 & .00 & .00 \\
\hline 25 & .00 & .00 & .00 & .00 & .00 & .00 & .00 & .02 & .00 & .00 & .00 & .00 \\
\hline 26 & e. 73 & .00 & .00 & .00 & .00 & .00 & .00 & .00 & .00 & .00 & .00 & .00 \\
\hline 27 & .00 & .08 & .00 & .00 & .00 & .00 & .00 & .00 & .00 & .00 & .00 & .00 \\
\hline 28 & .00 & .00 & .33 & .00 & .00 & .44 & .00 & .07 & .00 & .00 & .00 & .00 \\
\hline 29 & e. 08 & .09 & .23 & .00 & .00 & .13 & .00 & .01 & .00 & .00 & .00 & .00 \\
\hline 30 & .00 & .00 & .01 & .00 & -- & .37 & .00 & .06 & .00 & .00 & .00 & .00 \\
\hline 31 & .00 & --- & .02 & .00 & --- & .15 & -- & .01 & --- & .13 & .00 & --- \\
\hline TOTAL & 0.81 & 0.21 & 0.99 & 1.74 & 3.91 & 3.73 & 0.30 & 0.34 & 0.18 & 1.30 & 0.32 & 1.10 \\
\hline $\begin{array}{l}\text { CAL YR } \\
\text { WTR YR }\end{array}$ & $\begin{array}{l}1991 \\
1992\end{array}$ & $\begin{array}{l}\text { TOTAL } \\
\text { TOTAL }\end{array}$ & & & & & & & & & & \\
\hline
\end{tabular}

e Estimated 
3 SPRINGS BASIN

KAWICH PEAK NEAR WARM SPRINGS, NV PRECIPITATION WATER QUALITY, WATER YEAR OCTOBER 1991 TO SEPTEMBER 1992

\begin{tabular}{|c|c|c|c|c|c|c|c|c|c|c|c|}
\hline DATE & TIME & $\begin{array}{l}\text { SPE- } \\
\text { CIFIC } \\
\text { CON- } \\
\text { DUCT- } \\
\text { ANCE } \\
\text { (US/CM) }\end{array}$ & $\begin{array}{c}\text { SPE- } \\
\text { CIFIC } \\
\text { CON- } \\
\text { DUCT- } \\
\text { ANCE } \\
\text { LAB } \\
\text { (US/CM) }\end{array}$ & $\begin{array}{c}\text { PH } \\
\text { WATER } \\
\text { WHOLE } \\
\text { FIELD } \\
\text { (STAND- } \\
\text { ARD } \\
\text { UNITS) }\end{array}$ & $\begin{array}{c}\text { PH } \\
\text { WATER } \\
\text { WHOLE } \\
\text { LAB } \\
\text { (STAND- } \\
\text { ARD } \\
\text { UNITS) }\end{array}$ & $\begin{array}{c}\text { ALKA- } \\
\text { IINITY } \\
\text { LAB } \\
\text { (HG/L } \\
\text { AS } \\
\text { CACO3) }\end{array}$ & $\begin{array}{l}\text { CALCIUM } \\
\text { DIS- } \\
\text { SOLVED } \\
\text { (MG/L } \\
\text { AS CA) }\end{array}$ & $\begin{array}{l}\text { MAGNE- } \\
\text { SIUM, } \\
\text { DIS- } \\
\text { SOLVED } \\
\text { (MG/L } \\
\text { AS MG) }\end{array}$ & $\begin{array}{l}\text { SODIUM, } \\
\text { DIS- } \\
\text { SOLVED } \\
\text { (MG/L } \\
\text { AS NA) }\end{array}$ & $\begin{array}{l}\text { POTAS- } \\
\text { SIUM, } \\
\text { DIS- } \\
\text { SOLVED } \\
\text { (MG/L } \\
\text { AS K) }\end{array}$ & $\begin{array}{c}\text { SULFATE } \\
\text { DIS- } \\
\text { SOLVED } \\
\text { (MG/L } \\
\text { AS SO4) }\end{array}$ \\
\hline $\begin{array}{l}\text { FEB } 1992 \\
18 \ldots \\
\text { APR }\end{array}$ & 1430 & 12 & 13 & 6.8 & 8.2 & 2.9 & 0.78 & 0.08 & 1.4 & 0.20 & 1.0 \\
\hline JUN & 1131 & 5 & 6 & 5.7 & 6.2 & $<0.5$ & 0.17 & 0.02 & $<0.20$ & $<0.10$ & 0.55 \\
\hline${ }_{\text {JUL }}^{09} \ldots$ & 1500 & 37 & 39 & 6.4 & 6.4 & 4.0 & 2.5 & 0.26 & 1.0 & 0.90 & 3.6 \\
\hline $\operatorname{SEP}_{\text {SEP }}^{28}$ & 1145 & 28 & 18 & 6.1 & 7.5 & 0.5 & 0.66 & 0.08 & 0.30 & 0.20 & 1.4 \\
\hline $17 \ldots$ & 0755 & 19 & 19 & 6.1 & 6.0 & 0.5 & 1.4 & 0.14 & 0.40 & 0.20 & 2.3 \\
\hline DATE & $\begin{array}{l}\text { CHLO- } \\
\text { RIDE, } \\
\text { DIS- } \\
\text { SOLVED } \\
\text { (MG/L } \\
\text { AS CL) }\end{array}$ & $\begin{array}{l}\text { FLUO- } \\
\text { RIDE, } \\
\text { DIS- } \\
\text { SOLVED } \\
\text { (MG/L } \\
\text { AS F) }\end{array}$ & $\begin{array}{c}\text { BROMIDE } \\
\text { DIS- } \\
\text { SOLVED } \\
\text { (MG/L } \\
\text { AS BR) }\end{array}$ & $\begin{array}{c}\text { SILICA, } \\
\text { DIS- } \\
\text { SOLVED } \\
\text { (MG/L } \\
\text { AS } \\
\text { SIO2) }\end{array}$ & $\begin{array}{l}\text { NITRO- } \\
\text { GEN, } \\
\text { NITRATE } \\
\text { DIS- } \\
\text { SOLVED } \\
\text { (MG/L } \\
\text { AS N) }\end{array}$ & $\begin{array}{l}\text { PHOS- } \\
\text { PHORUS } \\
\text { ORTHO, } \\
\text { DIS- } \\
\text { SOLVED } \\
\text { (MG/L } \\
\text { AS P) }\end{array}$ & $\begin{array}{l}\text { IRON, } \\
\text { DIS- } \\
\text { SOLVED } \\
\text { (UG/L } \\
\text { AS FE) }\end{array}$ & $\begin{array}{l}\text { MANGA- } \\
\text { NESE, } \\
\text { DIS- } \\
\text { SOLVED } \\
\text { (UG/I } \\
\text { AS MN) }\end{array}$ & $\begin{array}{l}\text { H-2 / } \\
\text { H-1 } \\
\text { STABLE } \\
\text { ISOTOPE } \\
\text { RATIO } \\
\text { PER } \\
\text { MIL }\end{array}$ & $\begin{array}{c}\text { O-18 } \\
\text { O-16 } \\
\text { STABLE } \\
\text { ISOTOPE } \\
\text { RATIO } \\
\text { PER } \\
\text { MIL }\end{array}$ & \\
\hline $\begin{array}{l}\text { FEB } 1992 \\
18 \ldots \\
\text { APR }\end{array}$ & 0.42 & 0.03 & $<0.010$ & 1.5 & 0.200 & $<0.010$ & 7 & 1 & -106.0 & -15.15 & \\
\hline$\underset{\text { JUN }}{08 \ldots}$ & 0.12 & $<0.01$ & $<0.010$ & $<0.10$ & 0.330 & $<0.010$ & $<3$ & 1 & -103.0 & -14.40 & \\
\hline $\begin{array}{c}09 \\
\text { JUL }\end{array}$ & 0.88 & 0.07 & $<0.010$ & 0.20 & 1.40 & 0.070 & $<3$ & 38 & -52.5 & -7.70 & \\
\hline${ }_{\text {SEP }}^{28} \cdots$ & 0.52 & 0.06 & $<0.010$ & $<0.10$ & 0.630 & 0.060 & $<3$ & 9 & -124.0 & -16.90 & \\
\hline $17 \ldots$ & 0.42 & 0.10 & $<0.010$ & $<0.10$ & 1.40 & $<0.010$ & $<3$ & 20 & -40.5 & -6.20 & \\
\hline
\end{tabular}


3 SPRINGS BASIN

3 SPRINGS CiREER NEAR LEDGE SPRING, NEAR WARM SPRINGS, NV

LOCATION. -- Lat 37.57'42\%, Iong 116.26'23\%, Nye County, Hydrologic Unit 16060012, $1.1 \mathrm{mi}$ upstream from the 3 Springs Creek surface-water equipment, $3.6 \mathrm{mi}$ upstream from Eden Ranch in the Reveille valley, and $16.3 \mathrm{miles}$ south of Warm Springs at the intersection of us Highway 6 and Nevada Highway 375 .

DRAINAGE AREA. -- $0.78 \mathrm{mi}^{2}$.

PERIOD OF RECORD. -- JulY 1985 to current year.

SURFACE-WATER QUALITY, WATER YEAR OCTOBER 1991 TO SEPTEMBER 1992

\begin{tabular}{|c|c|c|c|c|c|c|c|c|c|c|c|c|}
\hline DATE & TIME & $\begin{array}{l}\text { SPE- } \\
\text { CIFIC } \\
\text { CON- } \\
\text { DUCT- } \\
\text { ANCE } \\
\text { (US/CM) }\end{array}$ & $\begin{array}{c}\text { SPE- } \\
\text { CIFIC } \\
\text { CON- } \\
\text { DUCT- } \\
\text { ANCE } \\
\text { LAB } \\
\text { (US/CM) }\end{array}$ & $\begin{array}{c}\text { PH } \\
\text { WATER } \\
\text { WHOLE } \\
\text { FIELD } \\
\text { (STAND- } \\
\text { ARD } \\
\text { UNITS) }\end{array}$ & $\begin{array}{c}\text { PH } \\
\text { WATER } \\
\text { WHOLE } \\
\text { LAB } \\
\text { (STAND- } \\
\text { ARD } \\
\text { UNITS) }\end{array}$ & $\begin{array}{cc} & L \\
& W \\
\text { TEMPER- } & T \\
\text { ATURE } \\
\text { WATER } \\
\text { (DEG C) }\end{array}$ & $\begin{array}{l}\text { ALKA- } \\
\text { LINITY } \\
\text { WAT WH } \\
\text { TOT FET } \\
\text { FIELD } \\
\text { MG/L AS } \\
\text { CACO3 }\end{array}$ & $\begin{array}{c}\text { ALKA- } \\
\text { LINITY } \\
\text { LAB } \\
\text { (MG/L } \\
\text { AS } \\
\text { CACO3) }\end{array}$ & $\begin{array}{l}\text { CALCIUM } \\
\text { DIS- } \\
\text { SOLVED } \\
\text { (MG/L } \\
\text { AS CA) }\end{array}$ & $\begin{array}{l}\text { MAGNE- } \\
\text { SIUM, } \\
\text { DIS- } \\
\text { SOLVED } \\
\text { (MG/L } \\
\text { AS MG) }\end{array}$ & $\begin{array}{l}\text { SODIUM, } \\
\text { DIS- } \\
\text { SOLVED } \\
\text { (MG/L } \\
\text { AS NA) }\end{array}$ & $\begin{array}{l}\text { POTAS- } \\
\text { SIUM, } \\
\text { DIS- } \\
\text { SOLVED } \\
\text { (MG/L } \\
\text { AS K) }\end{array}$ \\
\hline $\begin{array}{c}\text { JUN } 1992 \\
09 . .\end{array}$ & 1615 & 92 & 99 & 6.4 & 7.6 & 7.5 & 37 & 36 & 7.9 & 1.6 & 8.1 & 2.7 \\
\hline DATE & $\begin{array}{c}\text { SULFATE } \\
\text { DIS- } \\
\text { SOLVED } \\
\text { (MG/L } \\
\text { AS SO4) }\end{array}$ & $\begin{array}{l}\text { CHLO- } \\
\text { RIDE, } \\
\text { DIS- } \\
\text { SOLVED } \\
\text { (MG/L } \\
\text { AS CL) }\end{array}$ & $\begin{array}{l}\text { FLUO- } \\
\text { RIDE, } \\
\text { DIS- } \\
\text { SOLVED } \\
\text { (MG/L } \\
\text { AS F) }\end{array}$ & $\begin{array}{c}\text { BROMIDE } \\
\text { DIS- } \\
\text { SOLVED } \\
\text { (MG/L } \\
\text { AS BR) }\end{array}$ & $\begin{array}{c}\text { SILICA, } \\
\text { DIS- } \\
\text { SOLVED } \\
\text { (MG/L } \\
\text { AS } \\
\text { SIO2) }\end{array}$ & $\begin{array}{c}\text { NITRO- } \\
\text { GEN, } \\
\text { NITRATE } \\
\text { DIS- } \\
\text { SOLVED } \\
\text { (MG/L } \\
\text { AS N) }\end{array}$ & $\begin{array}{c}\text { PHOS- } \\
\text { PHORUS } \\
\text { ORTHO, } \\
\text { DIS- } \\
\text { D SOLVED } \\
\text { (MG/L } \\
\text { AS P) }\end{array}$ & $\begin{array}{l}\text { IRON, } \\
\text { DIS- } \\
\text { SOLVED } \\
\text { (UG/L } \\
\text { AS FE) }\end{array}$ & $\begin{array}{l}\text { MANGA- } \\
\text { NESE, } \\
\text { DIS- } \\
\text { SOLVED } \\
\text { (UG/L } \\
\text { AS MN) }\end{array}$ & $\begin{array}{c}\text { H-2 / } \\
\text { H-1 } \\
\text { STABLE } \\
\text { ISOTOPE } \\
\text { RATIO } \\
\text { PER } \\
\text { MIL }\end{array}$ & $\begin{array}{c}\text { 0-18 } \\
0-16 \\
\text { OTTABLE } \\
\text { STOTOPE } \\
\text { ISOTIO } \\
\text { RATIO } \\
\text { PER } \\
\text { MIL }\end{array}$ & \\
\hline
\end{tabular}

JUN 1992

$09 . .$.

5.3

4.3

0.16

0.040

46

$0.030<0.010$

$<3$

$<1 \quad-103.0 \quad-14.25$ 


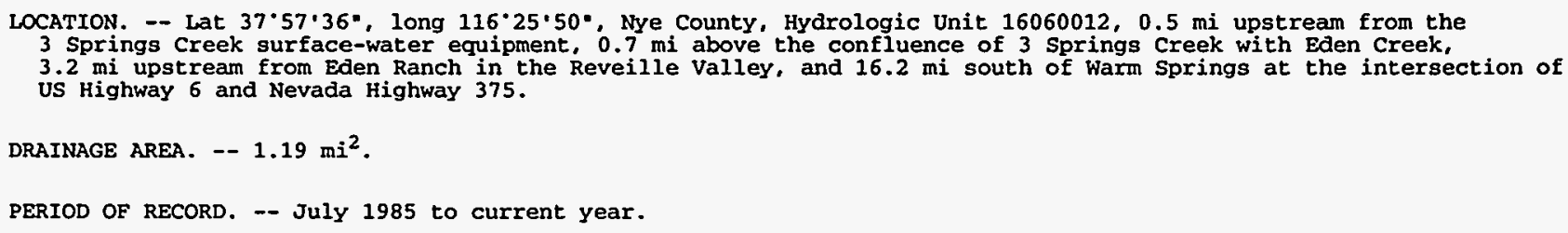

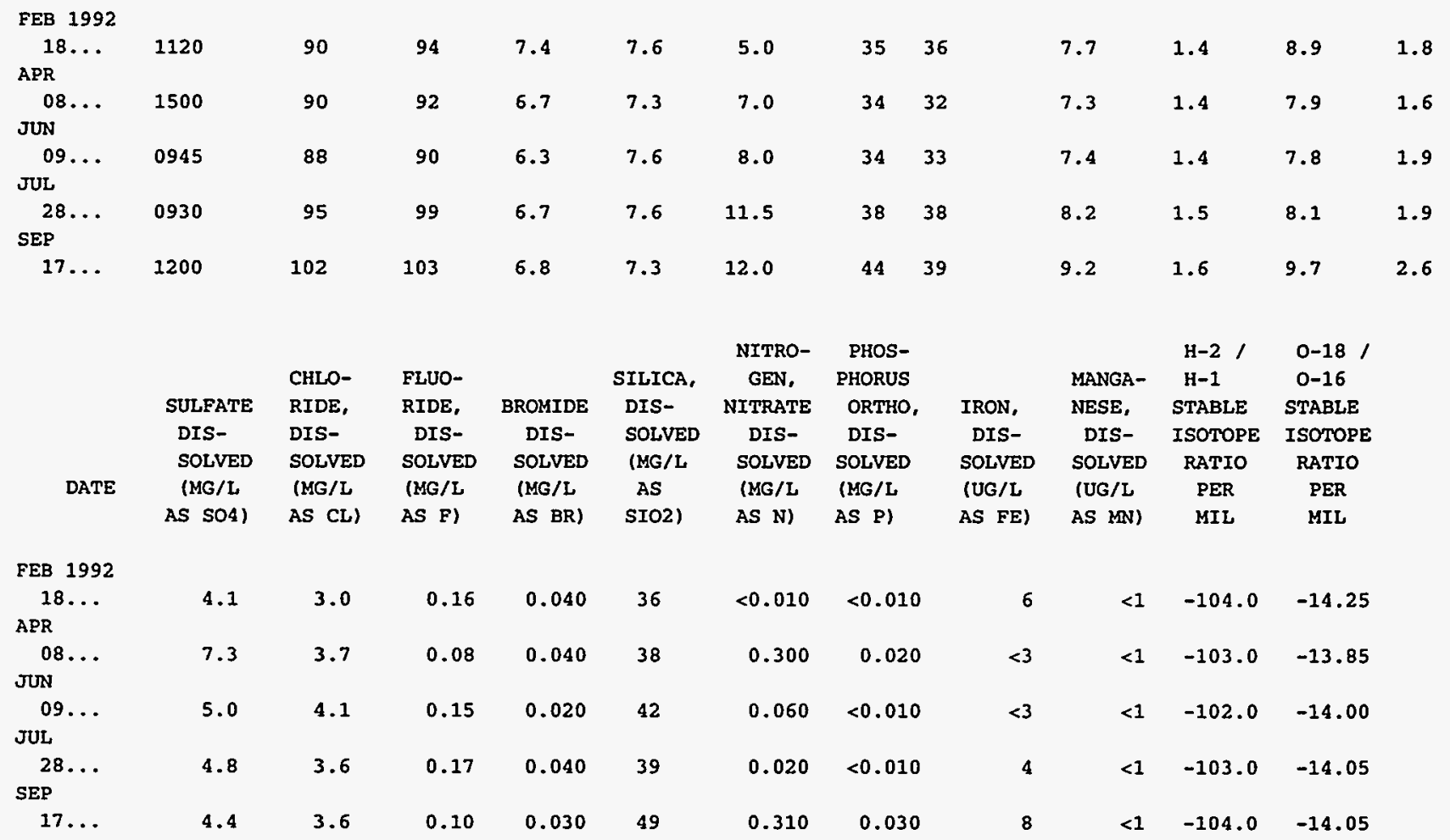


3 SPRINGS BASIN

\section{SPRINGS CREEK NEAR WARM SPRINGS, NV}

LOCATION. -- Lat 37.57'38\%, long 116.25'20\%, Nye County, Hydrologic Unit 16060012, on the left bank 0.2 $\mathrm{mi}$ above the confluence of 3 Springs Creek with Eden Creek, $2.7 \mathrm{mi}$ upstream from Eden Ranch in the Reveille Valley, and $16.1 \mathrm{mi}$ south of Warm Springs at the intersection of US Highway 6 and Nevada Highway 375 .

DRAINAGE AREA, -- $1.62 \mathrm{mi}^{2}$.

PERIOD OF RECORD. -- October 1985 to current year.

GAGE. -- Two water-stage recorders on a. 6 in Parshall flume and a standard 90 degree v-notch weir, and a crest-stage gage. Altitude of gage is $7,070 \mathrm{ft}$ above sea level, from topographic map. The flume is considered the primary gage. See REMARKS for more information.

REMARKS.-- The weather equipment is located $0.1 \mathrm{mi}$ downstream from the surface-water equipment. The surface-water-quality samples are taken upstream from the Parshall flume. The unsaturated-zone waterquality samples are taken approximately $0.1 \mathrm{mi}$ north and uphill from the meteorological site. Daily discharge values are determined from the 6 in Parshall flume. Accuracy of measurement by the flume diminishes below a discharge of $0.16 \mathrm{ft}^{3} / \mathrm{s}$. Daily discharges below $0.16 \mathrm{ft}^{3} / \mathrm{s}$ are considered fair; discharges above $0.16 \mathrm{ft}^{3} / \mathrm{s}$ are considered good. Estimated periods of daily record are considered poor. Values for the daily maximum and minimum air temperature were obtained from the forty minute average values.

PERIOD OF DAILY RECORD. --

AIR TEMPERATURE: October 1985 to current year.

SOIL TEMPERATURE: October 1985 to current year. SOLAR RADIATION: October 1985 to current year.

RELATIVE HUMIDITY: October 1990 to November 1991, February 1992 to current year.

TOTAL PRECIPITATION: October 1985 to current year.

DISCHARGE: October 1986 to current year.

EXTREMES FOR PERIOD OF RECORD. --

AIR TEMPERATURE: Maximum daily, $32.2^{\circ} \mathrm{C}$, July 7, 1989 and July 4, 1991; minimum daily, -28.8 ${ }^{\circ} \mathrm{C}$, Feb. 6, 1989. See REMARKS for more information.

SOIL TEMPERATURE: Maximum daily mean, $31^{\circ} 7^{\circ} \mathrm{C}$, July 7,1990 ; minimum daily mean, $-7^{.} 4^{\circ} \mathrm{C}$

Dec. 30, 1990 .

SOLAR RADIATION: Maximum daily total, $827 \mathrm{cal} / \mathrm{cm}^{2}$, June 18, 1989; minimum daily total.

$3 \mathrm{Cal} / \mathrm{cm}^{2}, \mathrm{Feb} 6,1992$.

RELATIVE HUMIDITY: Maximum daily mean, 99.1 pct, Jan 4, 1991; minimum daily mean, 12.5 pct, July 5, 1991 and July 29, 1992.

TOTAL PRECIPITATION: Maximum daily, 1.50 in, Nov. 1, 1987; no precipitation, most days of record. DISCHARGE: Maximum daily mean, $2.1 \mathrm{ft}^{3} / \mathrm{s}$, Apr. 29-30, 1988; no flow, most days of record.

EXTREMES FOR CURRENT YEAR. --

AIR TEMPERATURE: Maximum daily, $30.8^{\circ} \mathrm{C}$, July 29; minimum daily, $-16.2^{\circ} \mathrm{C}$, Jan. 13 . See REMARKS for more information.

SOIL TEMPERATURE: Maximum daily mean, $27.3^{\circ} \mathrm{C}$, Aug. 5; minimum daily mean, $-2.8^{\circ} \mathrm{C}$, DeG. 22 and Dec. $25-26$. SOLAR RADIATION: Maximum daily total, $754 \mathrm{cal} / \mathrm{cm}^{2}$, July 29 ; minimum daily total, $3 \mathrm{cal}^{2} \mathrm{~cm}^{2}$, Feb 6. RELATIVE HUMIDITY: Maximum daily mean, 98.2 pct, Oct. 27; minimum daily mean, 12.5 pct. July 29. TOTAL PRECIPITATION: Maximum daily, 1.09 in, July 11; no precipitation, most days of record. DISCHARGE: Maximum daily mean, $0.52 \mathrm{ft}^{3} / \mathrm{s}$, Apr. 15; no flow, most days of record. 
3 SPRINGS BASIN

3 SPRINGS CREEK NEAR WARM SPRINGS, NV

DAILY MEAN AIR TEMPERATURE, WATER YEAR OCTOBER 1991 TO SEPTEMBER 1992 DEGREES CELSIUS

\begin{tabular}{|c|c|c|c|c|c|c|c|c|c|c|c|c|}
\hline DAY & OCT & NOV & DEC & JAN & FEB & MAR & APR & MAY & JUN & JUL & AUG & SEP \\
\hline $\begin{array}{l}1 \\
2 \\
3 \\
4 \\
5\end{array}$ & $\begin{array}{l}13.1 \\
12.7 \\
13.1 \\
11.5 \\
14.4\end{array}$ & $\begin{array}{r}.6 \\
1.4 \\
2.3 \\
4.0 \\
6.4\end{array}$ & $\begin{array}{r}-8.3 \\
-.5 \\
-4.0 \\
-2.2 \\
-2.5\end{array}$ & $\begin{array}{l}-7.9 \\
-6.8 \\
-3.7 \\
-3.1 \\
-1.4\end{array}$ & $\begin{array}{r}2.2 \\
-.4 \\
-2.7 \\
-3.3 \\
-.9\end{array}$ & $\begin{array}{r}3.7 \\
.6 \\
1.9 \\
2.7 \\
2.6\end{array}$ & $\begin{array}{l}5.6 \\
6.0 \\
9.9 \\
9.6 \\
7.7\end{array}$ & $\begin{array}{r}9.7 \\
9.4 \\
12.0 \\
13.2 \\
11.8\end{array}$ & $\begin{array}{l}19.2 \\
19.2 \\
18.5 \\
20.3 \\
18.5\end{array}$ & $\begin{array}{l}11.2 \\
14.8 \\
19.7 \\
20.9 \\
21.1\end{array}$ & $\begin{array}{l}21.0 \\
25.2 \\
24.5 \\
23.0 \\
20.8\end{array}$ & $\begin{array}{l}14.8 \\
15.5 \\
16.5 \\
12.2 \\
14.1\end{array}$ \\
\hline $\begin{array}{r}6 \\
7 \\
8 \\
9 \\
10\end{array}$ & $\begin{array}{l}17.3 \\
17.9 \\
14.7 \\
11.8 \\
11.8\end{array}$ & $\begin{array}{l}7.1 \\
7.1 \\
9.9 \\
8.9 \\
5.8\end{array}$ & $\begin{array}{r}.5 \\
1.4 \\
-3.9 \\
-4.0 \\
-2.4\end{array}$ & $\begin{array}{r}-5.9 \\
-8.2 \\
-11.1 \\
-6.5 \\
-2.8\end{array}$ & $\begin{array}{r}-.8 \\
.1 \\
1.2 \\
1.5 \\
-.3\end{array}$ & $\begin{array}{r}.9 \\
-2.0 \\
1.7 \\
2.5 \\
2.4\end{array}$ & $\begin{array}{r}7.8 \\
8.0 \\
10.6 \\
12.5 \\
11.3\end{array}$ & $\begin{array}{l}10.7 \\
13.0 \\
12.8 \\
11.7 \\
11.3\end{array}$ & $\begin{array}{l}14.2 \\
14.9 \\
15.2 \\
19.1 \\
18.7\end{array}$ & $\begin{array}{l}21.3 \\
19.2 \\
19.0 \\
20.3 \\
20.9\end{array}$ & $\begin{array}{l}18.9 \\
21.9 \\
22.6 \\
23.5 \\
22.1\end{array}$ & $\begin{array}{l}15.7 \\
15.3 \\
18.7 \\
17.5 \\
19.5\end{array}$ \\
\hline $\begin{array}{l}11 \\
12 \\
13 \\
14 \\
15\end{array}$ & $\begin{array}{l}14.0 \\
13.7 \\
11.9 \\
11.6 \\
12.7\end{array}$ & $\begin{array}{r}2.3 \\
3.2 \\
4.8 \\
-1.1 \\
-1.8\end{array}$ & $\begin{array}{l}-4.8 \\
-1.1 \\
-2.3 \\
-3.6 \\
-2.6\end{array}$ & $\begin{array}{l}-3.9 \\
-9.9 \\
-7.9 \\
-6.6 \\
-7.6\end{array}$ & $\begin{array}{r}-.2 \\
.0 \\
-.9 \\
-2.5 \\
-2.4\end{array}$ & $\begin{array}{l}3.0 \\
4.8 \\
7.3 \\
6.6 \\
5.4\end{array}$ & $\begin{array}{r}12.4 \\
12.1 \\
11.8 \\
8.6 \\
6.1\end{array}$ & $\begin{array}{l}15.8 \\
16.2 \\
15.7 \\
16.6 \\
15.7\end{array}$ & $\begin{array}{r}17.9 \\
13.1 \\
8.1 \\
5.5 \\
5.0\end{array}$ & $\begin{array}{l}16.6 \\
13.2 \\
14.8 \\
17.9 \\
17.8\end{array}$ & $\begin{array}{l}23.6 \\
24.5 \\
18.9 \\
21.1 \\
21.6\end{array}$ & $\begin{array}{l}19.4 \\
17.1 \\
15.2 \\
18.0 \\
17.7\end{array}$ \\
\hline $\begin{array}{l}16 \\
17 \\
18 \\
19 \\
20\end{array}$ & $\begin{array}{r}17.1 \\
12.7 \\
12.4 \\
10.4 \\
6.9\end{array}$ & $\begin{array}{r}-1.2 \\
2.6 \\
-.7 \\
-2.9 \\
2.6\end{array}$ & $\begin{array}{r}-3.2 \\
1.2 \\
2.9 \\
-4.1 \\
-6.5\end{array}$ & $\begin{array}{l}-1.6 \\
-2.2 \\
-5.2 \\
-7.3 \\
-5.5\end{array}$ & $\begin{array}{r}-4.5 \\
-5.4 \\
-1.4 \\
2.0 \\
4.7\end{array}$ & $\begin{array}{l}2.5 \\
-.8 \\
-.8 \\
1.9 \\
2.2\end{array}$ & $\begin{array}{r}8.0 \\
11.8 \\
4.9 \\
5.0 \\
9.0\end{array}$ & $\begin{array}{l}16.2 \\
16.8 \\
16.7 \\
14.9 \\
12.6\end{array}$ & $\begin{array}{r}9.9 \\
13.9 \\
15.6 \\
16.2 \\
15.9\end{array}$ & $\begin{array}{l}19.2 \\
19.9 \\
19.4 \\
19.9 \\
20.1\end{array}$ & $\begin{array}{l}20.7 \\
22.8 \\
23.2 \\
24.9 \\
23.3\end{array}$ & $\begin{array}{r}17.8 \\
17.1 \\
9.9 \\
12.6 \\
15.6\end{array}$ \\
\hline $\begin{array}{l}21 \\
22 \\
23 \\
24 \\
25\end{array}$ & $\begin{array}{r}9.1 \\
11.7 \\
6.6 \\
1.0 \\
5.7\end{array}$ & $\begin{array}{r}1.6 \\
-6.8 \\
-5.5 \\
1.5 \\
3.8\end{array}$ & $\begin{array}{l}-5.0 \\
-4.5 \\
-2.2 \\
-3.9 \\
-3.5\end{array}$ & $\begin{array}{r}-5.0 \\
-5.2 \\
-3.4 \\
.0 \\
3.1\end{array}$ & $\begin{array}{r}5.7 \\
4.4 \\
.5 \\
1.5 \\
2.7\end{array}$ & $\begin{array}{l}1.8 \\
1.2 \\
1.2 \\
2.3 \\
3.3\end{array}$ & $\begin{array}{r}6.8 \\
8.1 \\
6.5 \\
10.0 \\
13.4\end{array}$ & $\begin{array}{l}10.5 \\
10.4 \\
12.9 \\
17.0 \\
19.0\end{array}$ & $\begin{array}{l}17.1 \\
19.1 \\
19.3 \\
18.4 \\
15.3\end{array}$ & $\begin{array}{l}19.7 \\
21.1 \\
19.8 \\
20.2 \\
21.3\end{array}$ & $\begin{array}{l}22.4 \\
17.8 \\
12.8 \\
14.5 \\
16.6\end{array}$ & $\begin{array}{r}16.1 \\
20.6 \\
19.7 \\
17.6 \\
8.3\end{array}$ \\
\hline $\begin{array}{l}26 \\
27 \\
28 \\
29 \\
30 \\
31\end{array}$ & $\begin{array}{r}3.9 \\
-4.8 \\
-6.1 \\
-1.8 \\
-6.9 \\
-4.4\end{array}$ & $\begin{array}{r}9.7 \\
4.0 \\
-5.1 \\
-5.8 \\
-9.3 \\
-.-\end{array}$ & $\begin{array}{l}-2.4 \\
-1.3 \\
-1.7 \\
-2.4 \\
-3.7 \\
-7.0\end{array}$ & $\begin{array}{r}-1.0 \\
-1.5 \\
.6 \\
-1.2 \\
-.1 \\
.1\end{array}$ & $\begin{array}{l}2.5 \\
3.8 \\
5.6 \\
6.7 \\
--- \\
---\end{array}$ & $\begin{array}{l}4.3 \\
3.6 \\
2.7 \\
3.9 \\
3.0 \\
4.0\end{array}$ & $\begin{array}{c}16.0 \\
18.1 \\
16.7 \\
16.8 \\
14.9 \\
\end{array}$ & $\begin{array}{l}18.1 \\
15.6 \\
13.7 \\
14.4 \\
14.7 \\
17.1\end{array}$ & $\begin{array}{r}16.9 \\
20.5 \\
21.6 \\
16.5 \\
15.1 \\
-.-\end{array}$ & $\begin{array}{l}22.8 \\
22.9 \\
24.2 \\
25.6 \\
24.8 \\
21.6\end{array}$ & $\begin{array}{l}14.5 \\
16.8 \\
20.9 \\
20.0 \\
16.4 \\
14.2\end{array}$ & $\begin{array}{r}9.9 \\
13.3 \\
14.3 \\
16.7 \\
18.2 \\
---\end{array}$ \\
\hline $\begin{array}{l}\text { MEAN } \\
\text { MAX } \\
\text { MIN } \\
\text { MED }\end{array}$ & $\begin{array}{r}8.9 \\
17.9 \\
-6.9 \\
11.8\end{array}$ & $\begin{array}{r}1.6 \\
9.9 \\
-9.3 \\
2.3\end{array}$ & $\begin{array}{r}-2.8 \\
2.9 \\
-8.3 \\
-2.6\end{array}$ & $\begin{array}{r}-4.2 \\
3.1 \\
-11.1 \\
-3.9\end{array}$ & $\begin{array}{r}.7 \\
6.7 \\
-5.4 \\
.1\end{array}$ & $\begin{array}{r}2.6 \\
7.3 \\
-2.0 \\
2.5\end{array}$ & $\begin{array}{r}10.2 \\
18.1 \\
4.9 \\
9.7\end{array}$ & $\begin{array}{r}14.1 \\
19.0 \\
9.4 \\
14.4\end{array}$ & $\begin{array}{r}16.0 \\
21.6 \\
5.0 \\
16.7\end{array}$ & $\begin{array}{l}19.7 \\
25.6 \\
11.2 \\
19.9\end{array}$ & $\begin{array}{l}20.5 \\
25.2 \\
12.8 \\
21.1\end{array}$ & $\begin{array}{r}15.8 \\
20.6 \\
8.3 \\
16.3\end{array}$ \\
\hline
\end{tabular}

CAL YR 1991 MEAN 7.5 MAX 25.6 MIN -9.8 MED 5.9 WTR YR 1992 MEAN 8.6 MAX 25.6 MIN -11.1 MED 9.8 
3 SPRINGS BASIN

3 SPRINGS CREEK.NEAR WARM SPRINGS, NV

DAILY MAXIMUM AIR TEMPERATURE, WATER YEAR OCTOBER 1991 TO SEPTEMBER 1992 DEGREES CELSIUS

\begin{tabular}{|c|c|c|c|c|c|c|c|c|c|c|c|c|}
\hline DAY & OCT & NOV & DEC & JAN & FEB & MAR & APR & MAY & JUN & JUL & AUG & SEP \\
\hline 1 & 23.8 & 10.6 & -3.2 & .5 & 4.8 & 5.7 & 12.3 & 16.5 & 27.1 & 18.8 & 29.4 & 22.4 \\
\hline 2 & 24.1 & 12.0 & 7.9 & 3.3 & 4.3 & 2.8 & 15.7 & 18.6 & 27.4 & 22.9 & 30.3 & 22.8 \\
\hline 3 & 24.4 & 12.7 & 5.9 & .0 & 2.5 & 5.4 & 17.6 & 20.8 & 27.4 & 24.9 & 30.5 & 19.9 \\
\hline 4 & 23.1 & 15.1 & 9.4 & -1.5 & 4.2 & 10.2 & 15.2 & 21.1 & 28.5 & 25.9 & 27.0 & 19.8 \\
\hline 5 & 24.9 & 17.6 & 10.3 & -.1 & 4.3 & 9.2 & 14.8 & 19.7 & 25.9 & 26.0 & 26.0 & 21.6 \\
\hline 6 & 24.1 & 18.3 & 10.2 & -3.9 & .0 & 3.4 & 14.4 & 17.9 & 20.3 & 26.7 & 24.9 & 23.1 \\
\hline 7 & 22.9 & 17.9 & 3.9 & -.3 & 1.5 & 2.4 & 15.4 & 21.2 & 23.3 & 25.4 & 26.0 & 24.5 \\
\hline 8 & 22.3 & 16.7 & -1.0 & -2.4 & 5.3 & 6.0 & 17.3 & 21.5 & 20.9 & 22.5 & 27.2 & 25.5 \\
\hline 9 & 21.3 & 14.7 & 3.9 & 3.6 & 2.9 & 8.0 & 17.5 & 17.7 & 24.0 & 27.1 & 29.7 & 27.2 \\
\hline 10 & 23.5 & 12.2 & 5.5 & 6.0 & 1.1 & 12.1 & 17.3 & 20.0 & 23.1 & 26.4 & 29.2 & 26.1 \\
\hline 11 & 23.6 & 13.5 & 3.0 & 5.4 & 1.4 & 12.4 & 17.1 & 21.9 & 22.7 & 21.3 & 30.7 & 23.3 \\
\hline 12 & 21.0 & 16.0 & 7.6 & -3.7 & .6 & 14.6 & 16.5 & 21.4 & 17.4 & 20.0 & 29.6 & 23.2 \\
\hline 13 & 22.3 & 14.8 & 5.1 & .2 & 2.4 & 12.7 & 16.1 & 20.7 & 15.3 & 21.5 & 28.9 & 23.5 \\
\hline 14 & 23.8 & 3.5 & 6.6 & .0 & .6 & 11.4 & 14.0 & 21.9 & 10.4 & 24.9 & 28.4 & 22.1 \\
\hline 15 & 25.4 & 1.9 & 7.6 & .6 & -1.6 & 9.6 & 13.7 & 22.1 & 11.5 & 24.7 & 29.2 & 22.2 \\
\hline 16 & 22.9 & 9.3 & 8.0 & 8.5 & 1.3 & 9.0 & 15.7 & 22.8 & 17.0 & 27.6 & 29.5 & 23.2 \\
\hline 17 & 22.4 & 7.1 & 6.3 & 3.2 & 1.8 & 5.2 & 17.8 & 22.1 & 22.0 & 29.8 & 30.3 & 23.0 \\
\hline 18 & 22.0 & 2.8 & 5.9 & .3 & 4.3 & 7.2 & 11.1 & 22.3 & 23.7 & 28.4 & 30.7 & 16.0 \\
\hline 19 & 19.8 & 6.5 & .1 & 3.8 & 9.5 & 9.5 & 13.5 & 18.9 & 24.5 & 28.9 & 30.8 & 21.7 \\
\hline 20 & 15.6 & 12.9 & -.5 & 4.4 & 12.1 & 8.2 & 17.6 & 17.9 & 25.3 & 28.5 & 28.9 & 23.9 \\
\hline 21 & 17.5 & 8.7 & 4.7 & 5.0 & 14.0 & 6.7 & 14.0 & 16.4 & 26.4 & 27.6 & 26.7 & 25.4 \\
\hline 22 & 15.1 & .4 & 4.1 & 7.1 & 10.1 & 4.0 & 15.0 & 19.0 & 26.6 & 25.2 & 23.1 & 25.5 \\
\hline 23 & 11.7 & 4.1 & 4.2 & 6.3 & 8.2 & 6.4 & 16.2 & 21.1 & 26.4 & 25.0 & 21.7 & 23.2 \\
\hline 24 & 8.3 & 12.9 & 4.4 & 11.2 & 12.4 & 9.0 & 19.6 & 23.5 & 24.1 & 25.5 & 23.1 & 24.6 \\
\hline 25 & 11.5 & 11.4 & 6.3 & 9.6 & 10.0 & 8.9 & 21.7 & 25.2 & 20.5 & 26.8 & 23.5 & 17.9 \\
\hline 26 & 8.5 & 13.9 & 5.9 & 6.5 & 12.5 & 9.0 & 21.8 & 23.8 & 24.5 & 28.8 & 25.1 & 21.3 \\
\hline 27 & .8 & 10.3 & 5.5 & 8.2 & 15.5 & 10.1 & 23.2 & 23.7 & 28.0 & 27.7 & 26.5 & 24.1 \\
\hline 28 & 1.8 & -.6 & -1.0 & 6.1 & 14.5 & 10.2 & 24.1 & 18.9 & 25.9 & 29.9 & 25.1 & 25.5 \\
\hline 29 & 3.3 & -2.3 & -1.3 & 7.6 & 11.3 & 10.3 & 21.9 & 21.5 & 20.7 & 30.8 & 24.2 & 24.6 \\
\hline 30 & -1.4 & -5.3 & .0 & 10.5 & -- & 7.1 & 19.4 & 21.8 & 20.8 & 30.2 & 20.9 & 23.5 \\
\hline 31 & 7.6 & $\ldots$ & 1.7 & 10.2 & -- & 11.5 & --- & 25.8 & -- & 29.0 & 22.1 & --- \\
\hline MAX & 25.4 & 18.3 & 10.3 & 11.2 & 15.5 & 14.6 & 24.1 & 25.8 & 28.5 & 30.8 & 30.8 & 27.2 \\
\hline
\end{tabular}

WTR YR $1992 \quad \mathrm{MAX} 30.8$ 
3 SPRINGS BASIN

3 SPRINGS CREEK NEAR WARM SPRINGS, NV

DAILY MINIMUM AIR TEMPERATURE, WATER YEAR OCTOBER 1991 TO SEPTEMBER 1992 DEGREES CELSIUS

\begin{tabular}{|c|c|c|c|c|c|c|c|c|c|c|c|c|}
\hline DAY & OCT & NOV & DEC & JAN & FEB & MAR & APR & MAY & JUN & JUL & AUG & SEP \\
\hline 1 & 5.2 & -7.4 & -14.5 & -14.1 & -2.8 & .7 & -1.6 & 1.5 & 6.5 & 4.0 & 10.0 & 5.4 \\
\hline 2 & 3.8 & -4.6 & -10.1 & -15.2 & -6.3 & -.4 & -2.9 & -.6 & 10.1 & 3.0 & 19.4 & 5.7 \\
\hline 3 & 3.0 & -3.7 & -10.2 & -6.3 & -8.7 & -.3 & -.8 & 1.0 & 6.5 & 14.6 & 16.0 & 11.1 \\
\hline 4 & 2.3 & -2.4 & -8.0 & -5.4 & -10.0 & -2.9 & 1.7 & 3.1 & 10.0 & 16.3 & 19.8 & 5.3 \\
\hline 5 & 4.4 & -.1 & -9.6 & -3.7 & -5.8 & -4.5 & -2.1 & 3.4 & 7.7 & 16.7 & 13.8 & 1.9 \\
\hline 6 & 7.1 & .4 & -8.2 & -10.4 & -2.6 & -4.0 & -.5 & 4.8 & 5.9 & 16.4 & 12.4 & 5.6 \\
\hline 7 & 11.6 & 1.5 & -3.0 & -14.7 & -1.0 & -6.5 & -1.6 & 2.9 & 3.7 & 9.3 & 18.1 & 2.4 \\
\hline 8 & 5.8 & 1.6 & -7.7 & -16.0 & -.6 & -1.1 & 1.2 & 5.6 & 9.3 & 14.7 & 19.0 & 10.1 \\
\hline 9 & 3.9 & 3.0 & -9.7 & -14.1 & .3 & -2.9 & 7.9 & 1.5 & 14.6 & 9.7 & 13.2 & 5.7 \\
\hline 10 & 2.1 & -.6 & -7.0 & -8.4 & -1.2 & -4.7 & 3.3 & -2.2 & 14.1 & 12.2 & 14.6 & 7.8 \\
\hline 11 & 5.4 & -4.5 & -9.2 & -15.5 & -3.3 & -3.2 & 6.0 & 4.3 & 11.8 & 7.3 & 12.3 & 14.1 \\
\hline 12 & 5.3 & -4.5 & -11.4 & -16.0 & -1.3 & -4.4 & 9.2 & 9.1 & 1.0 & 9.3 & 16.5 & 6.5 \\
\hline 13 & 4.0 & -3.2 & -8.8 & -16.2 & -3.7 & 1.2 & 7.7 & 7.6 & -1.9 & 8.5 & 12.7 & 3.4 \\
\hline 14 & 1.7 & -4.6 & -9.4 & -11.1 & -8.4 & .2 & 2.3 & 10.9 & -1.9 & 8.1 & 12.0 & 14.5 \\
\hline 15 & 2.3 & -4.7 & -7.7 & -14.2 & -4.8 & 1.9 & -1.6 & 3.9 & -2.7 & 12.1 & 13.3 & 13.7 \\
\hline 16 & 9.4 & -8.3 & -9.2 & -7.4 & -11.6 & -2.3 & -1.1 & 5.3 & .6 & 8.1 & 10.5 & 9.5 \\
\hline 17 & 3.9 & -2.8 & -8.0 & -8.2 & -13.2 & -5.6 & 5.0 & 9.2 & 1.4 & 12.3 & 13.3 & 10.2 \\
\hline 18 & 4.1 & -8.4 & .6 & -13.0 & -8.9 & -7.4 & -3.2 & 9.2 & 5.7 & 9.0 & 11.9 & 4.0 \\
\hline 19 & 1.8 & -9.0 & -7.3 & -14.0 & -4.1 & -6.2 & -3.9 & 9.0 & 3.8 & 10.6 & 15.6 & 3.0 \\
\hline 20 & -.3 & -5.2 & -11.7 & -11.6 & -1.3 & -2.4 & -1.6 & 5.7 & 5.3 & 10.3 & 13.5 & 8.2 \\
\hline 21 & -2.1 & -8.3 & -12.4 & -11.1 & -2.1 & -2.2 & .3 & 3.8 & 4.4 & 7.4 & 17.7 & 6.6 \\
\hline 22 & 8.7 & -12.0 & -10.1 & -13.4 & -.8 & -2.8 & .4 & .9 & 5.9 & 17.9 & 10.2 & 15.4 \\
\hline 23 & -3.4 & -12.6 & -7.5 & -10.5 & -7.6 & -1.7 & -2.7 & 1.6 & 10.0 & 11.2 & 2.5 & 13.7 \\
\hline 24 & -6.0 & -6.7 & -9.3 & -7.7 & -7.7 & -4.2 & -1.0 & 6.5 & 13.2 & 11.3 & 1.3 & 5.4 \\
\hline 25 & -.7 & -4.2 & -8.9 & -5.9 & -3.5 & -2.5 & 2.2 & 12.2 & 6.2 & 15.9 & 5.9 & -.4 \\
\hline 26 & -3.6 & 6.7 & -7.4 & -7.2 & -5.3 & -.7 & 5.5 & 10.8 & 7.9 & 18.3 & 3.2 & -.5 \\
\hline 27 & -9.7 & -8.2 & -6.8 & -7.9 & -4.0 & -1.4 & 13.8 & 4.3 & 8.5 & 16.0 & 4.1 & 4.7 \\
\hline 28 & -12.4 & -9.9 & -3.2 & -5.6 & -3.1 & -2.7 & 4.7 & 8.9 & 16.4 & 16.5 & 16.0 & 3.7 \\
\hline 29 & -6.2 & -9.5 & -3.2 & -7.6 & 2.1 & -1.8 & 8.7 & 6.5 & 12.2 & 20.6 & 13.3 & 6.9 \\
\hline 30 & -12.4 & -15.0 & -11.1 & -7.2 & --- & .7 & 9.6 & 6.7 & 9.9 & 16.8 & 12.0 & 10.1 \\
\hline 31 & -13.1 & $\cdots$ & -12.5 & -7.5 & --- & -.1 & --- & 8.5 & --- & 12.6 & 5.2 & -- \\
\hline MIN & -13.1 & -15.0 & -14.5 & -16.2 & -13.2 & -7.4 & -3.9 & -2.2 & -2.7 & 3.0 & 1.3 & -.5 \\
\hline
\end{tabular}

WTR YR 1992 MIN -16.2 
3 SPRINGS BASIN

3 SPRINGS CREEK NEAR WARM SPRINGS, NV

DAILY MEAN SOIL TEMPERATURE, WATER YEAR OCTOBER 1991 TO SEPTEMBER 1992 DEGREES CELSIUS

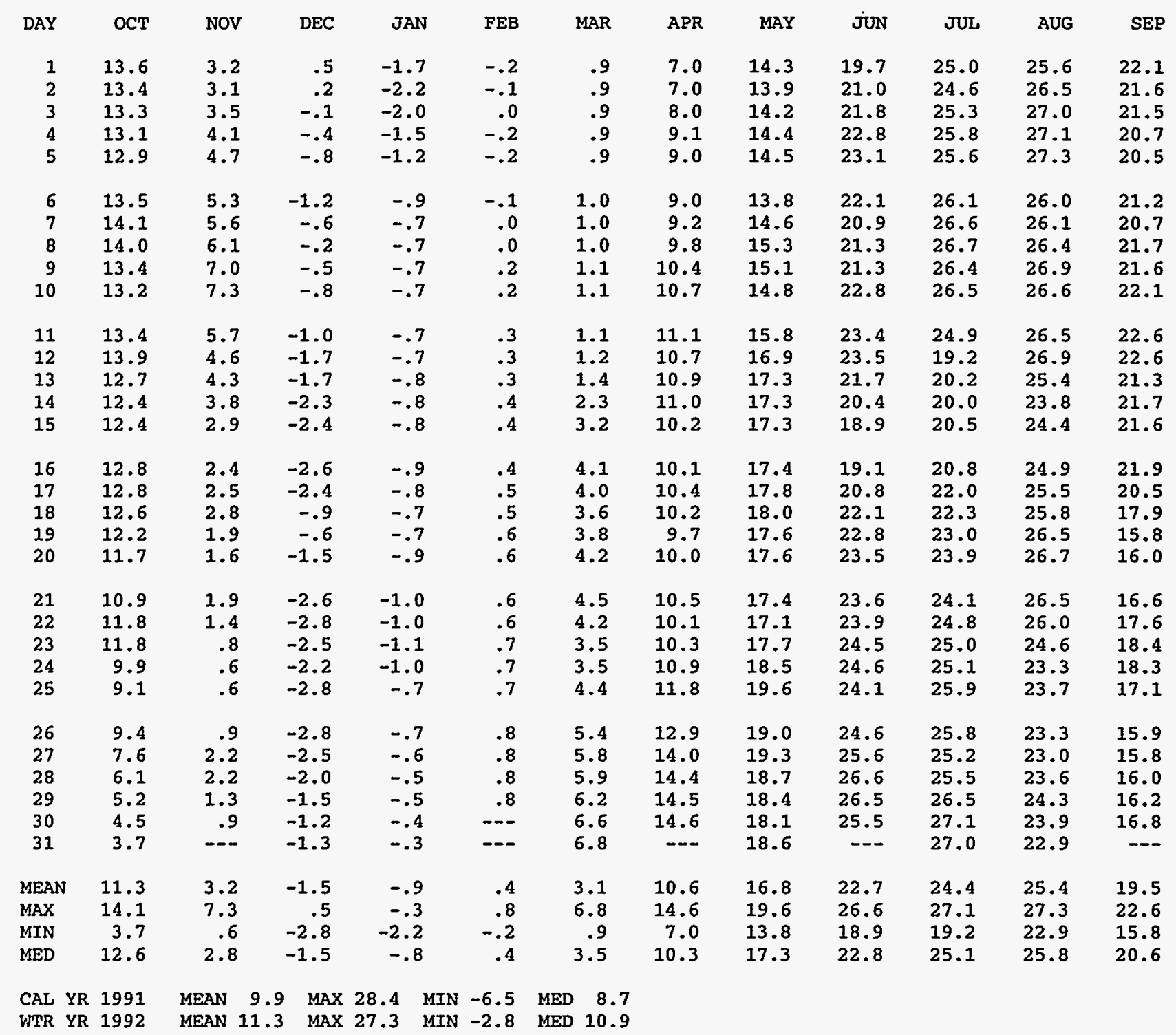


3 SPRINGS BASIN

3 SPRINGS CREEK NEAR WARM SPRINGS, NV

DAILY TOTAL SOLAR RADIATION, INCIDENTAI, WATER YEAR OCTOBER 1991 TO SEPTEMBER 1992 CALORIES PER SQUARE CENTIMETER

\begin{tabular}{|c|c|c|c|c|c|c|c|c|c|c|c|c|}
\hline DAY & OCT & NOV & $\mathrm{DEC}$ & JAN & FEB & MAR & APR & MAY & JUN & JUL & AUG & SEP \\
\hline 1 & 461 & 289 & 198 & 207 & 152 & 213 & 474 & 572 & 583 & 605 & 643 & e 426 \\
\hline 2 & 457 & 297 & 226 & 181 & 265 & 30 & 556 & 594 & 545 & 743 & 696 & e421 \\
\hline 3 & 450 & 286 & 223 & 56 & 295 & 223 & 577 & 612 & 708 & 549 & 704 & e 305 \\
\hline 4 & 452 & 291 & 222 & 91 & 232 & 390 & 471 & 514 & 700 & 703 & 577 & e541 \\
\hline 5 & 439 & 293 & 224 & 31 & 145 & 399 & 572 & 447 & 722 & 465 & 599 & e592 \\
\hline 6 & 416 & 295 & 214 & 175 & 3 & 193 & 558 & 385 & 389 & 573 & 484 & e497 \\
\hline 7 & 381 & 292 & 63 & 203 & 10 & 132 & 576 & 647 & 610 & 686 & 676 & e 606 \\
\hline 8 & 361 & 242 & 120 & 221 & 186 & 218 & 585 & 411 & 362 & 492 & 715 & e 592 \\
\hline 9 & 342 & 229 & 209 & 225 & 130 & 383 & 501 & 688 & 708 & 559 & 665 & e589 \\
\hline 10 & 412 & 206 & 201 & 222 & 92 & 456 & 452 & 688 & 728 & 512 & 477 & e567 \\
\hline 11 & 358 & 284 & 208 & 202 & 168 & 460 & 491 & 678 & 725 & 268 & 510 & e481 \\
\hline 12 & 249 & 275 & 207 & 231 & 53 & 468 & 489 & 611 & 677 & 388 & 417 & e 512 \\
\hline 13 & 401 & 239 & 206 & 203 & 112 & 438 & 597 & 626 & 609 & 372 & 337 & e 588 \\
\hline 14 & 407 & 114 & 198 & 230 & 158 & 335 & 406 & 670 & 469 & 425 & 621 & e588 \\
\hline 15 & 405 & 104 & 161 & 228 & 51 & 469 & 307 & 586 & 335 & 432 & 626 & e567 \\
\hline 16 & 398 & 267 & 200 & 237 & 334 & 364 & 511 & 621 & 738 & 528 & 613 & e509 \\
\hline 17 & 388 & 228 & 122 & 165 & 325 & 315 & 415 & 710 & 701 & 558 & 597 & 448 \\
\hline 18 & 382 & 216 & 178 & 238 & 298 & 404 & 639 & 688 & 733 & 552 & 671 & 305 \\
\hline 19 & 374 & 254 & 162 & 252 & 334 & 420 & 645 & 498 & 730 & 705 & 660 & 513 \\
\hline 20 & 374 & 255 & 205 & 253 & 331 & 280 & 609 & 725 & 684 & 711 & 667 & 512 \\
\hline 21 & 368 & 252 & 202 & 254 & 285 & 266 & 371 & 418 & 686 & 708 & 674 & 514 \\
\hline 22 & 325 & 249 & 186 & 263 & 354 & 200 & 602 & 691 & 561 & 711 & 620 & 500 \\
\hline 23 & 351 & 245 & 161 & 263 & 393 & 321 & 605 & 592 & 441 & 716 & 663 & 368 \\
\hline 24 & 238 & 177 & 200 & 265 & 391 & 357 & 572 & 612 & 591 & 696 & 645 & 501 \\
\hline 25 & 318 & 201 & 202 & 178 & 392 & 316 & 600 & 510 & 506 & 695 & 638 & 500 \\
\hline 26 & 197 & 217 & 207 & 183 & 404 & 323 & 590 & 484 & 654 & 459 & 661 & 496 \\
\hline 27 & 184 & 215 & 205 & 273 & 409 & 322 & 658 & 683 & 728 & 402 & 661 & 488 \\
\hline 28 & 336 & 225 & 58 & 94 & 410 & 258 & 641 & 296 & 698 & 662 & 493 & 482 \\
\hline 29 & 199 & 134 & 66 & 281 & 359 & 412 & 558 & 471 & 614 & 754 & 530 & 399 \\
\hline 30 & 321 & 229 & 65 & 276 & $\rightarrow$ & 189 & 636 & 368 & 627 & 732 & e 630 & 396 \\
\hline 31 & 321 & --- & 201 & 291 & -- & 282 & -- & 557 & --- & 466 & e 450 & -- \\
\hline TOTAL & 11065 & 7100 & 5500 & 6472 & 7071 & 9836 & 16264 & 17653 & 18562 & 17827 & 18620 & 14803 \\
\hline CAL YR & 1991 & TOTAL & 149594 & & & & & & & & & \\
\hline WTR YR & R 1992 & TOTAI & 150773 & & & & & & & & & \\
\hline
\end{tabular}

e Estimated 
3 SPRINGS BASIN

3 SPRINGS CREEK NEAR WARM SPRINGS, NV

DAILY MEAN RELATIVE HUMIDITY, WATER YEAR OCTOBER 1991 TO SEPTEMBER 1992 PERCENT

\begin{tabular}{|c|c|c|c|c|c|c|c|c|c|c|c|c|}
\hline DAY & $O C T$ & NOV & DEC & JAN & FEB & MAR & APR & MAY & JUN & JUL & AUG & SEP \\
\hline 1 & 50 & 72 & -- & -- & -- & 52 & 53 & 46 & 26 & 53 & 49 & 35 \\
\hline 2 & 40 & 81 & --- & -- & --- & 95 & 43 & 43 & 30 & 36 & 24 & 32 \\
\hline 3 & 32 & 83 & $-\cdots$ & -- & -- & 96 & 37 & 29 & 33 & 18 & 19 & 22 \\
\hline 4 & 25 & 81 & -- & --- & --- & 80 & 50 & 31 & 21 & 15 & 23 & 49 \\
\hline 5 & 23 & 77 & -- & -- & --- & 73 & 36 & 59 & 17 & 15 & 39 & 31 \\
\hline 6 & 22 & 79 & -- & --- & --- & 79 & 34 & 75 & 54 & 18 & 50 & 19 \\
\hline 7 & 19 & 80 & -. & --- & --- & 90 & 45 & 57 & 40 & 22 & 23 & 19 \\
\hline 8 & 27 & 66 & -- & $\cdots$ & $\cdots$ & 76 & 39 & 54 & 43 & 42 & 15 & 17 \\
\hline 9 & 32 & 69 & --- & --- & $-\cdots$ & 64 & 32 & 31 & 27 & 37 & 15 & 17 \\
\hline 10 & 30 & 86 & -- & -- & -- & 64 & 34 & 27 & 19 & 32 & 19 & 16 \\
\hline 11 & 25 & 66 & -- & --- & --- & 53 & 28 & 28 & 23 & 62 & 23 & 16 \\
\hline 12 & 36 & 48 & -- & -- & --- & 52 & 19 & 34 & 22 & 86 & 27 & 16 \\
\hline 13 & 34 & 45 & -- & $-\cdots$ & --- & 42 & 28 & 27 & 26 & 73 & 57 & 18 \\
\hline 14 & 25 & 58 & --- & --- & --- & 43 & 56 & 17 & 43 & 53 & 50 & 15 \\
\hline 15 & 25 & 69 & -- & -- & --- & 38 & 70 & 27 & 59 & 51 & 40 & 19 \\
\hline 16 & 17 & 69 & --- & -- & --- & 68 & 60 & 24 & 49 & 46 & 39 & 32 \\
\hline 17 & 25 & 51 & -- & --- & --- & 84 & 44 & 18 & 35 & 46 & 31 & 36 \\
\hline 18 & 28 & 61 & --- & -- & --- & 68 & 39 & 20 & 21 & 44 & 22 & 86 \\
\hline 19 & 37 & 60 & --- & -- & 80 & 57 & 35 & 22 & 26 & 32 & 15 & 59 \\
\hline 20 & 45 & --- & --- & --- & 68 & 59 & 30 & 23 & 24 & 21 & 15 & 37 \\
\hline 21 & 40 & $-\cdots$ & -- & -- & 51 & 87 & 63 & 46 & $=17$ & 19 & 14 & 36 \\
\hline 22 & 48 & $-\cdots$ & --- & -- & 59 & 91 & 33 & 54 & 17 & 16 & 19 & 30 \\
\hline 23 & 50 & $-\cdots$ & -- & --- & 49 & 91 & 37 & 43 & 27 & 16 & 17 & 37 \\
\hline 24 & 48 & -- & -- & --- & 45 & 81 & 35 & 35 & 29 & 17 & 17 & 27 \\
\hline 25 & 45 & --- & --- & $\cdots$ & 70 & 74 & 31 & 33 & 37 & 28 & 18 & 39 \\
\hline 26 & 72 & --- & --- & --- & 57 & 68 & 24 & 33 & 38 & 17 & 17 & 32 \\
\hline 27 & 98 & --- & --- & -- & 58 & 76 & 18 & 28 & 28 & 17 & 16 & 23 \\
\hline 28 & 82 & -- & -- & --- & 44 & 89 & 21 & 42 & 19 & 14 & 15 & 22 \\
\hline 29 & 75 & -- & $\cdots$ & --- & 32 & 80 & 17 & 62 & 34 & 12 & 17 & 22 \\
\hline 30 & 71 & --- & -- & --- & --- & 93 & 25 & 56 & 31 & 14 & 23 & 25 \\
\hline 31 & 63 & -- & --- & -- & --- & 90 & -- & 39 & -- & 37 & 33 & -- \\
\hline MEAN & 42 & -- & --- & --- & -- & 73 & 37 & 38 & 30 & 33 & 26 & 29 \\
\hline MAX & 98 & --- & -- & -- & --- & 96 & 70 & 75 & 59 & 86 & 57 & 86 \\
\hline MIN & 17 & --- & -- & -- & --- & 38 & 17 & 17 & 17 & 12 & 14 & 15 \\
\hline MED & 36 & --- & -- & -- & -- & 76 & 35 & 33 & 28 & 28 & 22 & 26 \\
\hline
\end{tabular}


3 SPRINGS BASIN

3 SPRINGS CREEK NEAR WARM SPRINGS, NV

DAILY TOTAL PRECIPITATION, WATER YEAR OCTOBER 1991 TO SEPTEMBER 1992 INCHES

\begin{tabular}{|c|c|c|c|c|c|c|c|c|c|c|c|c|}
\hline DAY & OCT & NOV & DEC & JAN & FEB & MAR & APR & MAY & JUN & JUL & AUG & SEP \\
\hline 1 & .00 & .00 & .00 & .00 & .00 & .00 & .02 & .00 & .00 & .00 & .00 & .00 \\
\hline 2 & .00 & .00 & .00 & .00 & .00 & .42 & .00 & .00 & .00 & .00 & .00 & .00 \\
\hline 3 & .00 & .00 & .00 & .11 & .00 & .20 & .00 & .00 & .00 & .00 & .00 & .00 \\
\hline 4 & .00 & .00 & .00 & .03 & .00 & .00 & .07 & .00 & .00 & .00 & .00 & .00 \\
\hline 5 & .00 & .00 & .00 & .93 & .00 & .00 & .00 & .15 & .00 & .00 & .02 & .00 \\
\hline 6 & .00 & .00 & .00 & .19 & .69 & .26 & .00 & .02 & .15 & .00 & .00 & .00 \\
\hline 7 & .00 & .00 & .14 & .02 & .11 & .14 & .00 & .00 & .00 & .00 & .00 & .00 \\
\hline 8 & .00 & .00 & .00 & .00 & .02 & .00 & .00 & .00 & .00 & .00 & .00 & .00 \\
\hline 9 & .00 & .00 & .00 & .00 & .02 & .00 & .00 & .00 & .00 & .00 & .00 & .00 \\
\hline 10 & .00 & .00 & .00 & .00 & .68 & .00 & .00 & .00 & .00 & .00 & .00 & .00 \\
\hline 11 & .00 & .00 & .00 & .00 & .26 & .00 & .00 & .00 & .00 & 1.09 & .00 & .00 \\
\hline 12 & .00 & .00 & .00 & .00 & .57 & .00 & .00 & .00 & .00 & .20 & .00 & .00 \\
\hline 13 & .00 & .00 & .00 & .00 & .10 & .00 & .00 & .00 & .00 & .16 & .38 & .00 \\
\hline 14 & .00 & .00 & .00 & .00 & .05 & .00 & .00 & .00 & .00 & .00 & .00 & .00 \\
\hline 15 & .00 & .00 & .00 & .00 & .65 & .00 & .05 & .00 & .00 & .00 & .00 & .00 \\
\hline 16 & .00 & .00 & .00 & .00 & .08 & .11 & .00 & .00 & .00 & .00 & .00 & .00 \\
\hline 17 & .00 & .02 & .00 & .00 & .00 & .05 & .00 & .00 & .00 & .05 & .00 & .68 \\
\hline 18 & .00 & .03 & .03 & .00 & .00 & .00 & .00 & .00 & .00 & .00 & .00 & .00 \\
\hline 19 & .00 & .00 & .04 & .00 & .00 & .00 & .00 & .00 & .00 & .00 & .00 & .00 \\
\hline 20 & .00 & .00 & .00 & .00 & .00 & .00 & .00 & .00 & .00 & .00 & .00 & .00 \\
\hline 21 & .00 & .00 & .00 & .00 & .10 & .05 & .10 & .00 & .00 & .00 & .00 & .00 \\
\hline 22 & .00 & .00 & .00 & .00 & .00 & .20 & .00 & .00 & .00 & .00 & .00 & .00 \\
\hline 23 & .00 & .00 & .00 & .00 & .00 & .52 & .00 & .00 & .00 & .00 & .00 & .00 \\
\hline 24 & .00 & .00 & .00 & .00 & .00 & .00 & .00 & .00 & .00 & .00 & .00 & .00 \\
\hline 25 & .00 & .00 & .00 & .00 & .00 & .00 & .00 & .05 & .00 & .00 & .00 & .00 \\
\hline 26 & .87 & .00 & .00 & .00 & .00 & .02 & .00 & .00 & .00 & .00 & .00 & .00 \\
\hline 27 & .03 & .14 & .00 & .00 & .00 & .00 & .00 & .00 & .00 & .00 & .00 & .00 \\
\hline 28 & .00 & .00 & .25 & .00 & .00 & .12 & .00 & .07 & .00 & .00 & .00 & .00 \\
\hline 29 & .10 & .03 & .15 & .00 & .00 & .12 & .00 & .03 & .00 & .00 & .00 & .00 \\
\hline 30 & .00 & .00 & .05 & .00 & --- & .28 & .00 & .00 & .00 & .00 & .00 & .00 \\
\hline 31 & .00 & $-\infty$ & .01 & .00 & --- & .17 & --- & .00 & --- & .06 & .00 & --- \\
\hline TOTAL & 1.00 & 0.22 & 0.67 & 1.28 & 3.33 & 2.66 & 0.24 & 0.32 & 0.15 & 1.56 & 0.40 & 0.68 \\
\hline
\end{tabular}

CAL YR 1991 TOTAL 14.40

WTR YR 1992 TOTAL 12.51 
3 SPRINGS BASIN

3 SPRINGS CREEK NEAR WARM SPRINGS, NV

PRECIPITATION WATER QUALITY, WATER YEAR OCTOBER 1991 TO SEPTEMBER 1992

\begin{tabular}{|c|c|c|c|c|c|c|c|c|c|c|c|}
\hline DATE & TIME & $\begin{array}{l}\text { SPE- } \\
\text { CIFIC } \\
\text { CON- } \\
\text { DUCT- } \\
\text { ANCE } \\
\text { (US/CM) }\end{array}$ & $\begin{array}{c}\text { SPE- } \\
\text { CIFIC } \\
\text { CON- } \\
\text { DUCT- } \\
\text { ANCE } \\
\text { LAB } \\
\text { (US/CM) }\end{array}$ & $\begin{array}{c}\text { PH } \\
\text { WATER } \\
\text { WHOLE } \\
\text { FIELD } \\
\text { (STAND- } \\
\text { ARD } \\
\text { UNITS) }\end{array}$ & $\begin{array}{c}\text { PH } \\
\text { WATER } \\
\text { WHOLE } \\
\text { LAB } \\
\text { (STAND- } \\
\text { ARD } \\
\text { UNITS) }\end{array}$ & $\begin{array}{c}\text { ALKA- } \\
\text { LINITY } \\
\text { LAB } \\
\text { (MG/L } \\
\text { AS } \\
\text { CACO3) }\end{array}$ & $\begin{array}{l}\text { CALCIUM } \\
\text { DIS- } \\
\text { SOLVED } \\
\text { (MG/L } \\
\text { AS CA) }\end{array}$ & $\begin{array}{l}\text { MAGNE- } \\
\text { SIUM, } \\
\text { DIS- } \\
\text { SOLVED } \\
\text { (MG/I, } \\
\text { AS MG) }\end{array}$ & $\begin{array}{l}\text { SODIUM, } \\
\text { DIS- } \\
\text { SOLVED } \\
\text { (MG/L } \\
\text { AS NA) }\end{array}$ & $\begin{array}{l}\text { POTAS- } \\
\text { SIUM, } \\
\text { DIS- } \\
\text { SOLVED } \\
\text { (MG/L } \\
\text { AS K) }\end{array}$ & $\begin{array}{l}\text { SULFATE } \\
\text { DIS- } \\
\text { SOLVED } \\
\text { (MG/L } \\
\text { AS SO4) }\end{array}$ \\
\hline \multirow{5}{*}{$\begin{array}{c}\text { FEB } 1992 \\
18 \ldots \\
\text { APR } \\
08 . \ldots \\
\text { JUN } \\
09 . \ldots \\
\text { JUL } \\
28 \ldots \\
\text { SEP } \ldots \\
17 \ldots\end{array}$} & 1015 & 5 & 7 & 6.4 & 8.1 & $<0.5$ & 0.33 & 0.03 & $<0.20$ & 0.10 & 0.50 \\
\hline & 1200 & 5 & 6 & 5.7 & 5.5 & $<0.5$ & 0.18 & 0.02 & $<0.20$ & $<0.10$ & 0.51 \\
\hline & 1845 & 33 & 33 & 6.3 & 6.1 & 3.1 & 2.1 & 0.25 & 0.80 & 0.80 & 3.1 \\
\hline & 1115 & 15 & 14 & 6.2 & 7.0 & 1.5 & 0.73 & 0.07 & 0.20 & 0.20 & 1.2 \\
\hline & 0930 & 17 & 17 & 5.4 & 5.5 & $<0.5$ & 1.2 & 0.12 & 0.20 & 0.20 & 1.9 \\
\hline DATE & $\begin{array}{l}\text { CHLO- } \\
\text { RIDE, } \\
\text { DIS- } \\
\text { SOLVED } \\
\text { (MG/L } \\
\text { AS CL) }\end{array}$ & $\begin{array}{l}\text { FLUO- } \\
\text { RIDE, } \\
\text { DIS- } \\
\text { SOLVED } \\
\text { (MG/L } \\
\text { AS F) }\end{array}$ & $\begin{array}{c}\text { BROMIDE } \\
\text { DIS- } \\
\text { SOLVED } \\
\text { (MG/L } \\
\text { AS BR) }\end{array}$ & $\begin{array}{l}\text { SILICA, } \\
\text { DIS- } \\
\text { SOLVED } \\
\text { (MG/L } \\
\text { AS } \\
\text { SIO2) }\end{array}$ & $\begin{array}{c}\text { NITRO- } \\
\text { GEN, } \\
\text { NITRATE } \\
\text { DIS- } \\
\text { SOLVED } \\
\text { (MG/L } \\
\text { AS N) }\end{array}$ & $\begin{array}{l}\text { PHOS- } \\
\text { PHORUS } \\
\text { ORTHO, } \\
\text { DIS- } \\
\text { SOLVED } \\
\text { (MG/L } \\
\text { AS P) }\end{array}$ & $\begin{array}{l}\text { IRON, } \\
\text { DIS- } \\
\text { SOLVED } \\
\text { (UG/L } \\
\text { AS EE) }\end{array}$ & $\begin{array}{l}\text { MANGA- } \\
\text { NESE, } \\
\text { DIS- } \\
\text { SOLVED } \\
\text { (UG/L } \\
\text { AS MN) }\end{array}$ & $\begin{array}{l}\text { H-2 / } \\
\text { H-1 } \\
\text { STABLE } \\
\text { ISOTOPE } \\
\text { RATIO } \\
\text { PER } \\
\text { MIL }\end{array}$ & $\begin{array}{l}\text { O-18 / } \\
\text { O-16 } \\
\text { STABLE } \\
\text { ISOTOPE } \\
\text { RATIO } \\
\text { PER } \\
\text { MIL }\end{array}$ & \\
\hline $\begin{array}{c}\text { FEB } 1992 \\
18 \ldots \\
\text { APR }\end{array}$ & 0.12 & 0.01 & $<0.010$ & $<0.10$ & 0.250 & $<0.010$ & 5 & 4 & -103.0 & -14.70 & \\
\hline$\underset{\text { JuN }}{08 \ldots}$ & 0.08 & $<0.01$ & $<0.010$ & $<0.10$ & 0.310 & $<0,010$ & 4 & 2 & -95.5 & -13.55 & \\
\hline $\begin{array}{l}09 \\
\text { JUL }\end{array}$ & 0.67 & 0.06 & $<0.010$ & 0.20 & 1.30 & 0.070 & $<3$ & 46 & -48.5 & -6.50 & \\
\hline & 0.28 & 0.05 & $<0.010$ & 0.10 & 0.770 & $<0.010$ & 4 & 13 & -123.0 & -16.75 & \\
\hline $17 \ldots$ & 0.32 & 0.07 & $<0.010$ & $<0.10$ & 1.40 & $<0.010$ & $<3$ & 21 & -33.5 & -5.00 & \\
\hline
\end{tabular}




\section{SPRINGS BASIN}

3 SPRINGS CREEK NEAR WARM SPRINGS, NV

WATER-QUALITY ANALYSIS FROM THE UNSATURATED-ZONE LYSIMETER, WATER YEAR OCTOBER 1991 TO SEPTEMBER 1992

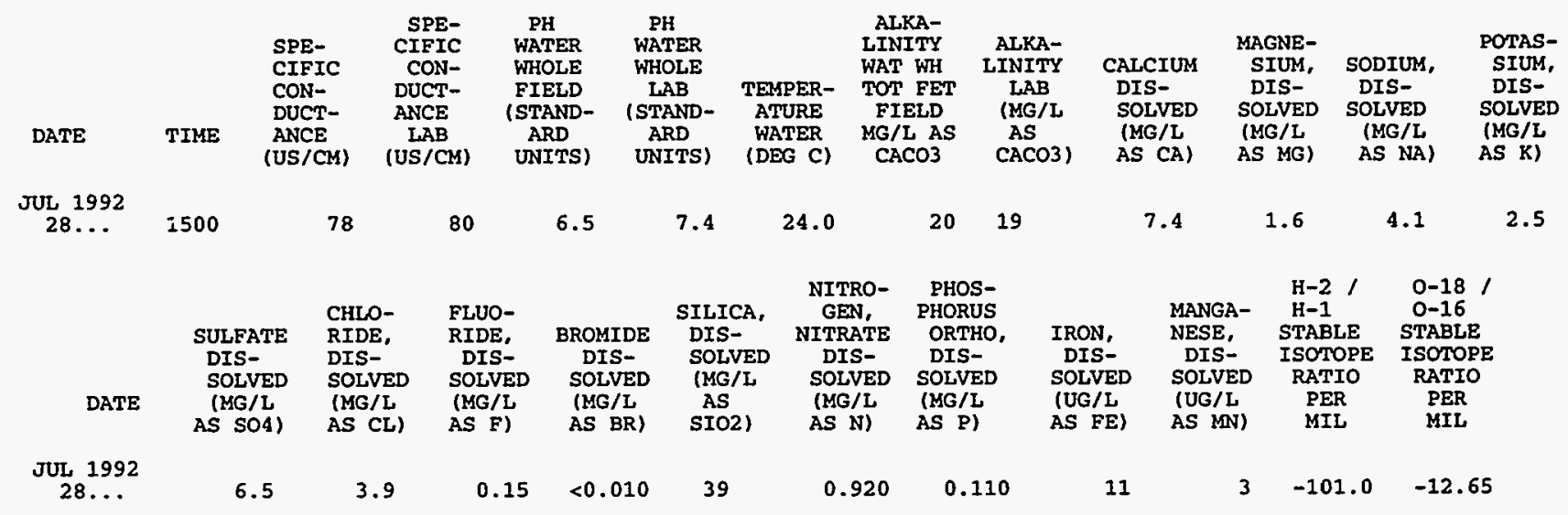


3 SPRINGS BASIN

3 SPRINGS CREEK NEAR WARM SPRINGS, NV

DAILY MEAN DISCHARGE, WATER YEAR OCTOBER 1991 TO SEPTEMBER 1992 CUBIC FEET PER SECOND

\begin{tabular}{|c|c|c|c|c|c|c|c|c|c|c|c|c|}
\hline DAY & OCT & NOV & DEC & JAN & FEB & MAR & APR & MAY & JUN & JUL & AUG & SEP \\
\hline 1 & .000 & .000 & .000 & .000 & .000 & .000 & .249 & .296 & .012 & .001 & .000 & .000 \\
\hline 2 & .000 & .000 & .000 & .000 & .000 & .000 & .264 & .289 & .012 & .001 & .000 & .000 \\
\hline 3 & .000 & .000 & .000 & .000 & .000 & .000 & .279 & .284 & .012 & .001 & .000 & .000 \\
\hline 4 & .000 & .000 & .000 & .000 & .000 & .000 & .295 & .283 & .012 & .001 & .000 & .000 \\
\hline 5 & .000 & .000 & .000 & .000 & .000 & .000 & .311 & .286 & .008 & .001 & .000 & .000 \\
\hline 6 & .000 & .000 & .000 & .000 & .000 & .000 & .327 & .285 & .008 & .001 & .000 & .000 \\
\hline 7 & .000 & .000 & .000 & .000 & .000 & .000 & .344 & .280 & .008 & .001 & .000 & .000 \\
\hline 8 & .000 & .000 & .000 & .000 & .000 & .000 & .364 & .278 & .008 & .001 & .000 & .000 \\
\hline 9 & .000 & .000 & .000 & .000 & .000 & .000 & .403 & .260 & .008 & .001 & .000 & .000 \\
\hline 10 & .000 & .000 & .000 & .000 & .000 & .000 & .437 & .230 & .008 & .001 & .000 & .000 \\
\hline 11 & .000 & .000 & .000 & .000 & .000 & .000 & .468 & .211 & .008 & .001 & .000 & .000 \\
\hline 12 & .000 & .000 & .000 & .000 & .000 & .000 & .485 & .190 & .008 & .001 & .000 & .000 \\
\hline 13 & .000 & .000 & .000 & .000 & .000 & .000 & .500 & .166 & .008 & .001 & .000 & .000 \\
\hline 14 & .000 & .000 & .000 & .000 & .000 & .000 & .508 & .141 & .008 & .001 & .000 & .000 \\
\hline 15 & .000 & .000 & .000 & .000 & .000 & .000 & .520 & .120 & .008 & .001 & .000 & .000 \\
\hline 16 & .000 & .000 & .000 & .000 & .000 & .000 & .516 & .104 & .008 & .000 & .000 & .000 \\
\hline 17 & .000 & .000 & .000 & .000 & .000 & .000 & .513 & .090 & .008 & .000 & .000 & .000 \\
\hline 18 & .000 & .000 & .000 & .000 & .000 & .003 & .506 & .080 & .008 & .000 & .000 & .000 \\
\hline 19 & .000 & .000 & .000 & .000 & .000 & .047 & .477 & .072 & .008 & .000 & .000 & .000 \\
\hline 20 & .000 & .000 & .000 & .000 & .000 & .071 & .468 & .065 & .008 & .000 & .000 & .000 \\
\hline 21 & .000 & .000 & .000 & .000 & .000 & .100 & .464 & .061 & .007 & .000 & .000 & .000 \\
\hline 22 & .000 & .000 & .000 & .000 & .000 & .118 & .451 & .053 & .004 & .000 & .000 & .000 \\
\hline 23 & .000 & .000 & .000 & .000 & .000 & .130 & .442 & .048 & .004 & .000 & .000 & .000 \\
\hline 24 & .000 & .000 & .000 & .000 & .000 & .135 & .431 & .043 & .004 & .000 & .000 & .000 \\
\hline 25 & .000 & .000 & .000 & .000 & .000 & .153 & .413 & .037 & .004 & .000 & .000 & .000 \\
\hline 26 & .000 & .000 & .000 & .000 & .000 & .166 & .394 & .033 & .004 & .000 & .000 & .000 \\
\hline 27 & .000 & .000 & .000 & .000 & .000 & .179 & .372 & .030 & .004 & .000 & .000 & .000 \\
\hline 28 & .000 & .000 & .000 & .000 & .000 & .192 & .352 & .029 & .004 & .000 & .000 & .000 \\
\hline 29 & .000 & .000 & .000 & .000 & .000 & .206 & .331 & .023 & .004 & .000 & .000 & .000 \\
\hline 30 & .000 & .000 & .000 & .000 & --- & .220 & .311 & .020 & .002 & .000 & .000 & .000 \\
\hline 31 & .000 & -- & .000 & .000 & --- & .234 & -- & .013 & --- & .000 & .000 & - \\
\hline TOTAL & 0.000 & 0.000 & 0.000 & 0.000 & 0.000 & 1.954 & 12.195 & 4.400 & 0.217 & 0.015 & 0.000 & 0.00 \\
\hline MEAN & .000 & .000 & .000 & .000 & .000 & .063 & .41 & .14 & .007 & .000 & .000 & .000 \\
\hline $\operatorname{MAX}$ & .000 & .000 & .000 & .000 & .000 & .234 & .520 & .296 & .012 & .001 & .000 & .00 \\
\hline MIN & .000 & .000 & .000 & .000 & .000 & .000 & .249 & .013 & .002 & .000 & .000 & .00 \\
\hline$A C-F T$ & .00 & .00 & .00 & .00 & .00 & 3.9 & 24 & 8.7 & .4 & .03 & .00 & .0 \\
\hline R & 1991 & TAL & & J. .005 & $\operatorname{MAX}$ & MIN & .000 & FT 3 . & & & & \\
\hline WTR & 92 & TAL & & $N .051$ & $\operatorname{MAX}$ & MIN & 100 & 37 & & & & \\
\hline
\end{tabular}


3 SPRINGS BASIN

3 SPRINGS CREEK NEAR WARM SPRINGS, NV SURFACE-WATER QUALITY, WATER YEAR OCTOBER 1991 TO SEPTEMBER 1992

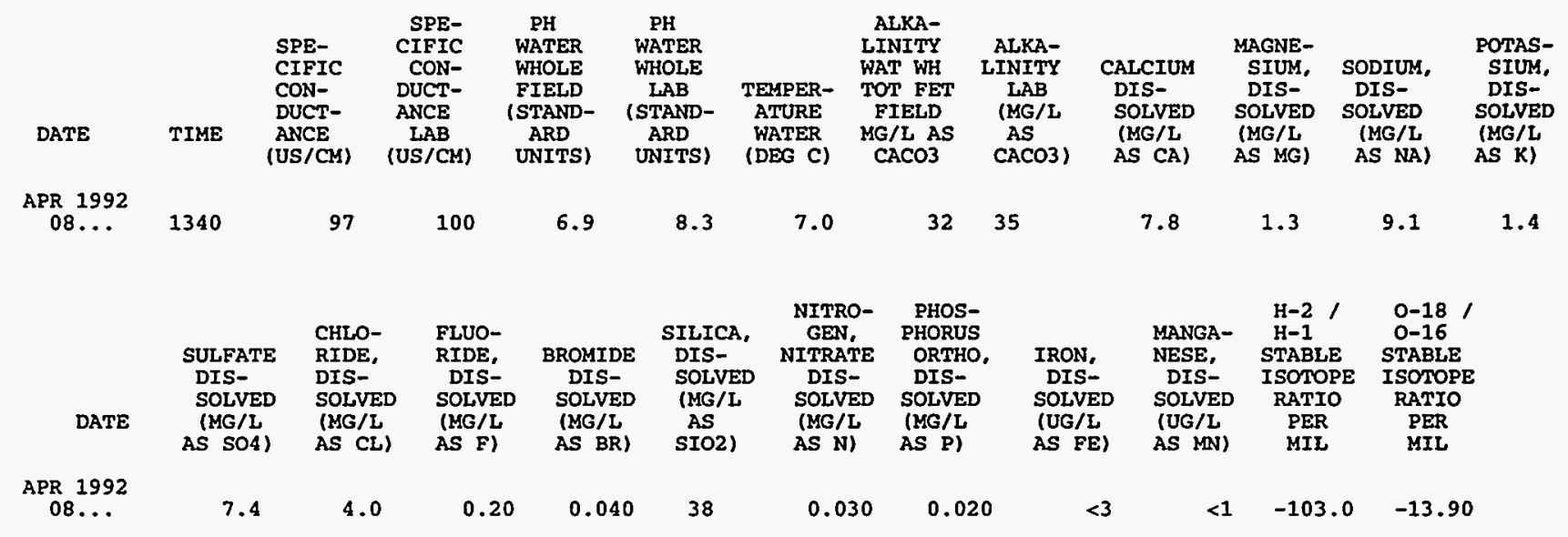




\section{RECORDS FROM EAST STEWART BASIN}


EAST STEWART BASIN

VEG SPRING NEAR IONE, NV

LOCATION. -- Lat $38^{\circ} 53^{\prime} 04^{*}$, long $17^{\circ} 21^{\prime} 13^{\prime}$. Nye County, Hydrologic Unit 16040107, on the right bank 0.5 $\mathrm{mi}$ upstream from the East Stewart Creek surface-water equipment, $1.8 \mathrm{mi}$ upstream from the Columbine campground, and $13.8 \mathrm{mi}$ east of Ione on State Route 21 .

PERIOD OF RECORD. -- September 1984 to current year.

REMARKS.-- The weather equipment is located approximately 200 ft southeast and uphill from Veg Spring. The two unsaturated-zone water-quality samples are taken approximately 20 ft downhill from the weather equipment. The maximum total daily precipitation was not published because the value is estimated. Estimated periods of daily record are considered poor. Values for the daily maximum and minimum air temperature were obtained from the forty minute average values.

PERIOD OF DAILY RECORD. --

AIR TEMPERATURE: October 1988 to current year.

SOIL TEMPERATURE: October 1988 to current year.

SOLAR RADIATION: November 1990 to current year.

RELATIVE HUMIDITY: October 1990 to current year.

TOTAL PRECIPITATION: October 1986 to current year.

EXTREMES FOR PERIOD OF RECORD. -

AIR TEMPERATURE: Maximum daily, 23.7 C, July 4, 1991; minimum daily, -27.0. C, Dec. $22,1990$.

See REMARKS for more information.

SOIL TEMPERATURE: Maximum daily mean, $19.9^{\circ} \mathrm{C}$, Aug. 6,1990 ; minimum daily mean, $-10.7^{\circ} \mathrm{C}$,

DeC. 30,1990

SOLAR RADIATION: Maximum daily total, $714 \mathrm{cal} / \mathrm{cm}^{2}$, May 15, 1991; minimum daily total,

$34 \mathrm{cal} / \mathrm{cm}^{2}$, Jan. 4, 1991.

RELATIVE HUMIDITY: Maximum daily mean, 100 pct, May 14, 1991 and May 30-31, 1991; minimum daily mean, 15.5 pct, Aug. 9, 1991 .

TOTAL PRECIPITATION: Maximum daily, not available; no precipitation, most days of record.

EXTREMES FOR CURRENT YEAR. --

AIR TEMPERATURE: Maximum daily, $23.6^{\circ} \mathrm{C}$, Aug. 1; minimum daily, $-19.2^{\circ} \mathrm{C}$, Nov. 30 . See REMARKS for more information.

SOIL TEMPERATURE: Maximum daily mean, $18.3^{\circ} \mathrm{C}$, Aug. 9; minimum daily mean, $-3.7^{\circ} \mathrm{C}$, Dec. $25-26$.

SOLAR RADIATION: Maximum daily total, $642 \mathrm{cal} / \mathrm{cm}^{2}$, July 4 ; minimum daily total, $46 \mathrm{cal} / \mathrm{cm}^{2}$, Jan. 5.

RELATIVE HUMIDITY: Maximum daily mean, 99.3 pct, Nov. 9; minimum daily mean, 16.5 pct, July 30 .

TOTAL PRECIPITATION: Maximum daily, 1.45 in, Jan. 5; no precipitation, most days of record. 
EAST STEWART BASIN

VEG SPRING NEAR IONE, NV

DAILY MEAN AIR TEMPERATURE, WATER YEAR OCTOBER 1991 TO SEPTEMBER 1992 DEGREES CELSIUS

\begin{tabular}{|c|c|c|c|c|c|c|c|c|c|c|c|c|}
\hline DAY & OCT & NOV & DEC & JAN & FEB & MAR & APR & MAY & JUN & JUL & AUG & SEP \\
\hline $\begin{array}{l}1 \\
2 \\
3 \\
4 \\
5\end{array}$ & $\begin{array}{r}8.8 \\
9.2 \\
9.3 \\
9.5 \\
10.6\end{array}$ & $\begin{array}{r}-3.1 \\
-2.2 \\
.4 \\
2.3 \\
3.4\end{array}$ & $\begin{array}{r}-8.4 \\
-4.7 \\
-1.2 \\
-.4 \\
1.9\end{array}$ & $\begin{array}{l}-3.2 \\
-2.0 \\
-3.9 \\
-4.7 \\
-6.6\end{array}$ & $\begin{array}{l}-5.3 \\
-7.7 \\
-8.5 \\
-6.0 \\
-5.5\end{array}$ & $\begin{array}{l}-3.8 \\
-4.9 \\
-3.7 \\
-4.3 \\
-3.3\end{array}$ & $\begin{array}{r}-.1 \\
2.8 \\
4.4 \\
1.5 \\
-1.4\end{array}$ & $\begin{array}{l}-.3 \\
3.4 \\
6.3 \\
8.1 \\
8.2\end{array}$ & $\begin{array}{l}12.7 \\
11.9 \\
12.5 \\
12.5 \\
10.0\end{array}$ & $\begin{array}{r}2.1 \\
7.8 \\
11.2 \\
12.2 \\
12.0\end{array}$ & $\begin{array}{l}15.9 \\
15.9 \\
15.1 \\
14.8 \\
13.9\end{array}$ & $\begin{array}{l}7.4 \\
9.5 \\
7.3 \\
2.3 \\
6.3\end{array}$ \\
\hline $\begin{array}{r}6 \\
7 \\
8 \\
9 \\
10\end{array}$ & $\begin{array}{r}11.2 \\
9.0 \\
8.6 \\
8.7 \\
9.6\end{array}$ & $\begin{array}{r}3.5 \\
5.2 \\
4.4 \\
.1 \\
-1.2\end{array}$ & $\begin{array}{r}2.3 \\
-4.8 \\
-8.4 \\
-2.6 \\
-4.2\end{array}$ & $\begin{array}{r}-11.7 \\
-11.3 \\
-6.4 \\
-.2 \\
.5\end{array}$ & $\begin{array}{l}-4.6 \\
-3.3 \\
-4.2 \\
-5.2 \\
-5.9\end{array}$ & $\begin{array}{l}-6.5 \\
-7.2 \\
-6.3 \\
-5.5 \\
-2.7\end{array}$ & $\begin{array}{r}-1.5 \\
1.2 \\
1.6 \\
2.4 \\
2.3\end{array}$ & $\begin{array}{l}6.3 \\
6.6 \\
7.5 \\
1.2 \\
5.2\end{array}$ & $\begin{array}{r}7.8 \\
8.1 \\
8.6 \\
11.0 \\
10.8\end{array}$ & $\begin{array}{l}10.9 \\
10.4 \\
11.5 \\
12.5 \\
12.3\end{array}$ & $\begin{array}{l}12.7 \\
14.6 \\
15.3 \\
16.2 \\
16.0\end{array}$ & $\begin{array}{r}7.0 \\
8.8 \\
10.4 \\
12.1 \\
12.7\end{array}$ \\
\hline $\begin{array}{l}11 \\
12 \\
13 \\
14 \\
15\end{array}$ & $\begin{array}{r}9.7 \\
8.3 \\
8.8 \\
10.1 \\
11.4\end{array}$ & $\begin{array}{r}2.3 \\
4.4 \\
-. .4 \\
-10.3 \\
-9.7\end{array}$ & $\begin{array}{r}-5.9 \\
-5.1 \\
-2.9 \\
1.5 \\
1.0\end{array}$ & $\begin{array}{r}-10.3 \\
-12.1 \\
-6.4 \\
-6.7 \\
-1.4\end{array}$ & $\begin{array}{l}-6.5 \\
-5.5 \\
-6.8 \\
-7.0 \\
-8.9\end{array}$ & $\begin{array}{r}-.5 \\
.3 \\
.2 \\
-1.5 \\
-2.8\end{array}$ & $\begin{array}{r}5.2= \\
4.2 \\
2.9 \\
.3 \\
-.3\end{array}$ & $\begin{array}{l}6.5 \\
7.1 \\
8.1 \\
7.6 \\
7.2\end{array}$ & $\begin{array}{r}10.3 \\
.6 \\
-1.2 \\
-4.9 \\
-3.2\end{array}$ & $\begin{array}{r}7.7 \\
7.1 \\
9.4 \\
10.7 \\
9.9\end{array}$ & $\begin{array}{l}17.0 \\
14.5 \\
14.3 \\
13.7 \\
13.2\end{array}$ & $\begin{array}{r}11.5 \\
7.7 \\
8.2 \\
9.5 \\
9.6\end{array}$ \\
\hline $\begin{array}{l}16 \\
17 \\
18 \\
19 \\
20\end{array}$ & $\begin{array}{l}8.8 \\
7.5 \\
7.2 \\
5.4 \\
2.3\end{array}$ & $\begin{array}{r}-2.6 \\
-3.5 \\
-9.6 \\
-3.2 \\
.7\end{array}$ & $\begin{array}{r}.3 \\
-1.1 \\
-4.8 \\
-12.7 \\
-4.8\end{array}$ & $\begin{array}{l}-1.8 \\
-6.2 \\
-7.2 \\
-4.2 \\
-4.0\end{array}$ & $\begin{array}{r}-11.2 \\
-9.5 \\
-6.1 \\
-2.3 \\
-2.0\end{array}$ & $\begin{array}{l}-5.9 \\
-7.6 \\
-6.4 \\
-3.4 \\
-4.2\end{array}$ & $\begin{array}{r}1.5 \\
1.5 \\
-4.5 \\
-1.8 \\
3.8\end{array}$ & $\begin{array}{l}8.4 \\
9.3 \\
8.8 \\
6.3 \\
1.5\end{array}$ & $\begin{array}{r}.5 \\
5.3 \\
6.3 \\
7.1 \\
8.7\end{array}$ & $\begin{array}{l}13.5 \\
12.5 \\
12.6 \\
12.5 \\
12.0\end{array}$ & $\begin{array}{l}13.5 \\
14.4 \\
16.0 \\
16.7 \\
14.3\end{array}$ & $\begin{array}{r}\text { e10.3 } \\
\text { e8.1 } \\
\text { e3.2 } \\
\text { e } 7.8 \\
\text { e9.2 }\end{array}$ \\
\hline $\begin{array}{l}21 \\
22 \\
23 \\
24 \\
25\end{array}$ & $\begin{array}{r}4.7 \\
3.8 \\
-4.4 \\
-4.9 \\
-3.3\end{array}$ & $\begin{array}{r}-5.4 \\
-5.3 \\
-.4 \\
-.2 \\
-.1\end{array}$ & $\begin{array}{l}-1.1 \\
-2.9 \\
-2.7 \\
-3.7 \\
-1.7\end{array}$ & $\begin{array}{r}-6.2 \\
-5.5 \\
-1.5 \\
.5 \\
.7\end{array}$ & $\begin{array}{r}-1.5 \\
-4.8 \\
-4.0 \\
-.3 \\
-1.2\end{array}$ & $\begin{array}{l}-3.9 \\
-4.5 \\
-5.3 \\
-3.3 \\
-2.0\end{array}$ & $\begin{array}{r}.5 \\
-2.3 \\
1.5 \\
5.8 \\
7.8\end{array}$ & $\begin{array}{r}3.4 \\
5.3 \\
8.4 \\
11.5 \\
11.5\end{array}$ & $\begin{array}{r}12.1 \\
13.5 \\
11.9 \\
9.9 \\
5.3\end{array}$ & $\begin{array}{l}12.2 \\
11.7 \\
10.1 \\
11.6 \\
12.8\end{array}$ & $\begin{array}{r}12.3 \\
6.4 \\
5.7 \\
8.0 \\
8.7\end{array}$ & $\begin{array}{r}\mathrm{e} 10.9 \\
\mathrm{e} 13.7 \\
\mathrm{e} 11.6 \\
\mathrm{e} 8.9 \\
\mathrm{e} 4.2\end{array}$ \\
\hline $\begin{array}{l}26 \\
27 \\
28 \\
29 \\
30 \\
31\end{array}$ & $\begin{array}{r}-4.3 \\
-11.0 \\
-11.5 \\
-9.2 \\
-10.4 \\
-1.3\end{array}$ & $\begin{array}{r}1.0 \\
-6.1 \\
-12.2 \\
-14.7 \\
-15.5 \\
-\ldots-\end{array}$ & $\begin{array}{l}-1.3 \\
-3.5 \\
-8.6 \\
-8.4 \\
-8.2 \\
-4.4\end{array}$ & $\begin{array}{r}-1.9 \\
-.9 \\
-3.2 \\
-.8 \\
1.9 \\
1.9\end{array}$ & $\begin{array}{r}1.2 \\
1.3 \\
.3 \\
-.9 \\
--- \\
---\end{array}$ & $\begin{array}{r}-2.7 \\
-1.3 \\
-.5 \\
-.8 \\
-1.9 \\
-2.1\end{array}$ & $\begin{array}{r}8.4 \\
8.4 \\
9.9 \\
10.2 \\
4.5 \\
---\end{array}$ & $\begin{array}{r}9.5 \\
8.2 \\
8.9 \\
8.5 \\
8.9 \\
11.1\end{array}$ & $\begin{array}{r}8.5 \\
13.4 \\
12.6 \\
5.2 \\
3.3 \\
-.-\end{array}$ & $\begin{array}{l}14.3 \\
15.3 \\
16.5 \\
16.5 \\
16.9 \\
15.0\end{array}$ & $\begin{array}{r}9.9 \\
12.2 \\
12.8 \\
8.5 \\
6.9 \\
6.2\end{array}$ & $\begin{array}{r}\mathrm{e} 6.8 \\
\mathrm{e} 9.2 \\
\mathrm{e} 10.8 \\
\mathrm{e} 12.6 \\
\mathrm{e} 11.9 \\
-\end{array}$ \\
\hline $\begin{array}{l}\text { MEAN } \\
\text { MAX } \\
\text { MIN } \\
\text { MED }\end{array}$ & $\begin{array}{r}3.9 \\
11.4 \\
-11.5 \\
8.3\end{array}$ & $\begin{array}{r}-2.6 \\
5.2 \\
-15.5 \\
-.8\end{array}$ & $\begin{array}{r}-3.6 \\
2.3 \\
-12.7 \\
-3.5\end{array}$ & $\begin{array}{r}-4.0 \\
1.9 \\
-12.1 \\
-3.9\end{array}$ & $\begin{array}{r}-4.5 \\
1.3 \\
-11.2 \\
-5.2\end{array}$ & $\begin{array}{r}-3.5 \\
.3 \\
-7.6 \\
-3.4\end{array}$ & $\begin{array}{r}2.7 \\
10.2 \\
-4.5 \\
2.0\end{array}$ & $\begin{array}{r}7.0 \\
11.5 \\
-.3 \\
7.6\end{array}$ & $\begin{array}{r}7.7 \\
13.5 \\
-4.9 \\
8.6\end{array}$ & $\begin{array}{r}11.7 \\
16.9 \\
2.1 \\
12.0\end{array}$ & $\begin{array}{r}12.8 \\
17.0 \\
5.7 \\
13.9\end{array}$ & $\begin{array}{r}9.0 \\
13.7 \\
2.3 \\
9.2\end{array}$ \\
\hline
\end{tabular}

CAL YR 1991 MEAN 1.9 MAX 17.8 MIN -15.5 MED 1

WTR YR 1992 MEAN 3.1 MAX 17.0 MIN -15.5 MED 2.3

e Estimated 
EAST STEWART BASIN

VEG SPRING NEAR IONE, NV

DAILY MAXIMUM AIR TEMPERATURE, WATER YEAR OCTOBER 1991 TO SEPTEMBER 1992 DEGREES CELSIUS

\begin{tabular}{|c|c|c|c|c|c|c|c|c|c|c|c|c|}
\hline DAY & OCT & NOV & $\mathrm{DEC}$ & JAN & FEB & MAR & APR & MAY & JUN & JUL & AUG & SEP \\
\hline 1 & 14.9 & .7 & -5.4 & 2.7 & -3.1 & 3.0 & 6.7 & 4.0 & 19.3 & 7.4 & 23.6 & 14.0 \\
\hline 2 & 15.5 & 2.2 & -.9 & 4.0 & -4.8 & -3.2 & 11.1 & 9.1 & 17.1 & 15.2 & 21.7 & 14.5 \\
\hline 3 & 15.5 & 4.6 & 5.2 & -1.0 & -5.8 & .5 & 13.0 & 13.4 & 18.3 & 17.0 & 20.3 & 13.1 \\
\hline 4 & 15.2 & 10.5 & 6.4 & -.4 & .7 & 4.1 & 6.0 & 14.2 & 17.3 & 17.9 & 21.2 & 6.2 \\
\hline 5 & 16.1 & 9.7 & 8.4 & -3.9 & -3.3 & 3.2 & 3.2 & 13.0 & 15.5 & 16.9 & 17.6 & 11.7 \\
\hline 6 & 16.4 & 9.0 & 5.8 & -10.8 & -2.5 & .3 & 3.8 & 10.5 & 12.1 & 15.6 & 17.4 & 11.0 \\
\hline 7 & 13.5 & 13.4 & 2.0 & -6.7 & 2.1 & -.5 & 6.6 & 12.6 & 13.6 & 17.3 & 20.4 & 14.4 \\
\hline 8 & 14.6 & 7.0 & -4.7 & -.7 & 3.0 & -4.3 & 7.3 & 12.2 & 14.3 & 16.2 & 20.2 & 15.7 \\
\hline 9 & 14.5 & 1.6 & 4.1 & 6.7 & .2 & -.2 & 6.7 & 6.4 & 17.2 & 19.1 & 22.7 & 18.3 \\
\hline 10 & 14.7 & 3.1 & 2.6 & 3.1 & -2.2 & 6.5 & 5.6 & 10.3 & 16.4 & 17.7 & 20.4 & 17.5 \\
\hline 11 & 14.0 & 9.3 & -2.0 & -.3 & -4.7 & 7.9 & 9.2 & 12.3 & 16.5 & 13.2 & 21.5 & 16.4 \\
\hline 12 & 13.2 & 10.0 & -1.0 & -6.5 & -4.2 & 8.9 & 8.2 & 11.7 & 7.0 & 12.2 & 18.7 & 12.6 \\
\hline 13 & 14.6 & 3.0 & 2.5 & -2.0 & -1.6 & 5.5 & 9.1 & 13.3 & 5.5 & 13.6 & 22.6 & 13.4 \\
\hline 14 & 15.8 & -8.1 & 6.3 & -3.2 & -.6 & 2.5 & 4.7 & 14.3 & -4.0 & 15.7 & 19.5 & 14.6 \\
\hline 15 & 18.1 & -7.1 & 3.4 & 6.3 & -5.8 & 4.9 & 6.7 & 13.5 & 2.1 & 13.5 & 18.5 & 15.9 \\
\hline 16 & 12.8 & 2.6 & 6.4 & 1.9 & -9.4 & -1.8 & 7.5 & 15.0 & 6.7 & 20.4 & 18.9 & --- \\
\hline 17 & 11.8 & .0 & 1.9 & -3.0 & -5.9 & -1.8 & 5.2 & 14.2 & 11.7 & 19.9 & 20.1 & $\cdots$ \\
\hline 18 & 12.8 & -8.2 & -2.3 & -4.9 & -2.2 & 1.0 & .7 & 13.9 & 11.6 & 17.3 & 22.0 & --- \\
\hline 19 & 11.0 & 4.5 & -9.3 & 1.0 & .5 & 1.9 & 5.7 & 12.6 & 12.3 & 19.6 & 21.7 & $\ldots$ \\
\hline 20 & 7.8 & 2.6 & 1.6 & 3.2 & .2 & -.8 & 10.2 & 7.4 & 14.2 & 17.3 & 19.4 & --- \\
\hline 21 & 9.4 & -1.4 & 7.1 & -1.4 & .7 & 2.0 & 4.2 & 9.1 & 17.7 & 19.0 & 18.4 & -- \\
\hline 22 & 5.9 & -.4 & 1.8 & -1.4 & -1.7 & .6 & 2.4 & 11.0 & 18.6 & 17.2 & 11.6 & --- \\
\hline 23 & -.3 & 7.9 & .7 & 3.2 & 2.4 & .2 & 9.7 & 13.6 & 16.0 & 15.9 & 13.7 & --- \\
\hline 24 & -2.7 & 2.7 & -.6 & 6.3 & 7.7 & 4.4 & 12.4 & 16.9 & 15.5 & 18.3 & 14.3 & $\cdots$ \\
\hline 25 & -1.1 & 3.5 & 4.2 & 2.5 & 5.7 & 2.6 & 14.4 & 17.8 & 9.3 & 18.3 & 15.1 & $\cdots$ \\
\hline 26 & -1.3 & 3.8 & 2.1 & .5 & 8.4 & 3.7 & 14.0 & 14.1 & 14.5 & 19.6 & 16.8 & --- \\
\hline 27 & -7.9 & 1.1 & .0 & 5.9 & 8.4 & 8.3 & 14.1 & 14.9 & 20.4 & 21.3 & 19.1 & -- \\
\hline 28 & -7.1 & -8.2 & -6.2 & .2 & 7.1 & 5.2 & 17.0 & 13.1 & 17.7 & 22.5 & 19.3 & --- \\
\hline 29 & -6.0 & -10.8 & -7.2 & 4.2 & 4.5 & 4.1 & 15.8 & 13.0 & 8.6 & 21.7 & 13.7 & --- \\
\hline 30 & -2.1 & -12.8 & -3.7 & 8.2 & --- & 2.3 & 10.7 & 13.8 & 9.3 & 23.2 & 13.0 & --- \\
\hline 31 & 5.4 & --- & 1.2 & 5.7 & -- & .3 & $-\infty$ & 17.5 & -- & 19.4 & 10.9 & --- \\
\hline $\operatorname{MAX}$ & 18.1 & 13.4 & 8.4 & 8.2 & 8.4 & 8.9 & 17.0 & 17.8 & 20.4 & 23.2 & 23.6 & -- \\
\hline
\end{tabular}

CAL YR 1991 MAX 23.7 
EAST STEWART BASIN

VEG SPRING NEAR IONE, NV

DAILY MINIMUM AIR TEMPERATURE, WATER YEAR OCTOBER 1991 TO SEPTEMBER 1992 DEGREES CELSIUS

\begin{tabular}{|c|c|c|c|c|c|c|c|c|c|c|c|c|}
\hline DAY & OCT & NOV & DEC & JAN & FEB & MAR & APR & MAY & JUN & JUL & AUG & SEP \\
\hline 1 & 5.2 & -4.9 & -13.6 & -5.3 & -8.5 & -5.7 & -4.4 & -4.7 & 8.0 & -2.1 & 11.3 & 4.6 \\
\hline 2 & 5.6 & -4.9 & -6.6 & -4.1 & -9.8 & -6.6 & -3.1 & -1.9 & 7.6 & .4 & 13.0 & 5.5 \\
\hline 3 & 5.9 & -1.9 & -3.3 & -7.1 & -10.2 & -6.2 & -.6 & 1.4 & 7.3 & 6.2 & 10.9 & -1.0 \\
\hline 4 & 6.0 & -1.0 & -2.0 & -7.6 & -9.3 & -7.5 & -3.3 & 3.5 & 8.6 & 8.0 & 10.2 & -1.9 \\
\hline 5 & 7.3 & 1.2 & -1.3 & -9.8 & -7.4 & -6.3 & -4.2 & 4.5 & 5.4 & 7.7 & 11.0 & 2.4 \\
\hline 6 & 7.7 & 1.6 & .5 & -12.3 & -5.6 & -10.3 & -4.7 & 3.8 & 4.5 & 6.9 & 9.5 & 4.1 \\
\hline 7 & 6.7 & 1.6 & -10.4 & -13.1 & -5.1 & -10.9 & -2.0 & 3.0 & 5.0 & 4.9 & 10.7 & 4.4 \\
\hline 8 & 4.9 & 1.2 & -11.1 & -12.6 & -6.6 & -7.6 & -2.1 & 3.8 & 4.3 & 7.0 & 11.5 & 6.5 \\
\hline 9 & 5.2 & -1.2 & -5.6 & -3.0 & -7.4 & -9.2 & -.3 & -3.4 & 7.3 & 7.0 & 10.9 & 7.1 \\
\hline 10 & 6.1 & -3.5 & -8.2 & -1.4 & -7.2 & -8.9 & -1.2 & -1.5 & 7.6 & 8.4 & 12.6 & 9.3 \\
\hline 11 & 7.3 & -1.6 & -7.3 & -14.6 & -7.5 & -4.4 & 2.5 & 1.6 & 6.0 & 4.2 & 13.7 & 6.8 \\
\hline 12 & 5.8 & 2.0 & -8.6 & -16.5 & -7.5 & -3.5 & .9 & 3.7 & -6.3 & 4.3 & 10.7 & 4.6 \\
\hline 13 & 5.7 & -8.0 & -6.0 & -8.9 & -9.6 & -2.2 & -.6 & 3.8 & -7.0 & 5.0 & 10.3 & 3.3 \\
\hline 14 & 6.8 & -12.6 & -.8 & -9.2 & -9.4 & -4.6 & -2.0 & 3.0 & -6.4 & 7.3 & 10.4 & 5.5 \\
\hline 15 & 8.5 & -11.9 & -.3 & -6.0 & -11.9 & -6.6 & -3.0 & 2.0 & -5.8 & 7.0 & 10.2 & 5.4 \\
\hline 16 & 5.4 & -7.6 & -2.0 & -4.2 & -13.6 & -8.4 & -2.7 & 3.7 & -3.7 & 8.9 & 9.7 & -- \\
\hline 17 & 4.9 & -8.5 & -3.1 & -9.9 & -11.5 & -10.0 & -5.0 & 5.7 & .0 & 8.2 & 10.3 & $-\infty$ \\
\hline 18 & 4.8 & -12.5 & -10.5 & -10.0 & -9.3 & -11.2 & -8.5 & 4.9 & 2.8 & 8.8 & 12.0 & --- \\
\hline 19 & .3 & -10.7 & -14.3 & -7.1 & -4.1 & -7.5 & -8.0 & -.9 & 2.9 & 7.3 & 11.5 & -- \\
\hline 20 & -.7 & -1.7 & -13.7 & -7.0 & -4.1 & -5.4 & -1.9 & -2.9 & 5.1 & 6.5 & 10.0 & -- \\
\hline 21 & 1.0 & -9.9 & -5.0 & -7.5 & -4.4 & -6.8 & -4.6 & -1.6 & 6.8 & 5.8 & 6.1 & --- \\
\hline 22 & .2 & -9.2 & -5.2 & -6.7 & -9.6 & -7.2 & -6.1 & -.3 & 10.1 & 5.9 & .9 & --- \\
\hline 23 & -7.2 & -3.4 & -4.5 & -6.4 & -9.1 & -9.1 & -4.9 & 2.9 & 6.9 & 4.8 & -1.2 & -- \\
\hline 24 & -7.5 & -2.8 & -5.3 & -2.8 & -3.6 & -8.9 & .7 & 6.4 & 4.2 & 6.0 & 3.9 & $-\infty$ \\
\hline 25 & -5.7 & -2.0 & -4.5 & -1.7 & -3.4 & -4.2 & 3.3 & 8.0 & 3.2 & 8.2 & 4.0 & --- \\
\hline 26 & -8.2 & -1.2 & -2.9 & -5.4 & -2.5 & -6.6 & 4.9 & 6.0 & 3.8 & 9.5 & 4.7 & --- \\
\hline 27 & -13.5 & -14.9 & -7.6 & -4.7 & -1.4 & -6.3 & 4.2 & 3.5 & 7.1 & 12.4 & 7.4 & -- \\
\hline 28 & -15.5 & -14.4 & -10.2 & -5.7 & -3.0 & -4.4 & 4.1 & 5.6 & 8.4 & 13.5 & 8.5 & --- \\
\hline 29 & -15.0 & -17.4 & -9.1 & -4.2 & -4.3 & -4.1 & 4.8 & 3.9 & -.1 & 12.1 & 3.9 & --- \\
\hline 30 & -16.0 & -19.2 & -9.7 & -.9 & $-\div-$ & -4.0 & -3.3 & 4.9 & -1.1 & 12.9 & 3.5 & -- \\
\hline 31 & -3.5 & -- & -7.4 & -3.3 & --- & -3.9 & $\cdots$ & 5.6 & --2 & 12.9 & 3.3 & --- \\
\hline MIN & -16.0 & -19.2 & -14.3 & -16.5 & -13.6 & -11.2 & -8.5 & -4.7 & -7.0 & -2.1 & -1.2 & --- \\
\hline
\end{tabular}

CAI YR 1991 MIN -19.2 
EAST STEWART BASIN

VEG SPRING NEAR IONE, NV

DAILY MEAN SOIL TEMPERATURE, WATER YEAR OCTOBER 1991 TO SEPTEMBER 1992 DEGREES CELSIUS

\begin{tabular}{|c|c|c|c|c|c|c|c|c|c|c|c|c|}
\hline DAY & OCT & NOV & DEC & JAN & FEB & MAR & APR & MAY & JUN & JUL & AUG & SEP \\
\hline $\begin{array}{l}1 \\
2 \\
3 \\
4 \\
5\end{array}$ & $\begin{array}{l}8.0 \\
8.3 \\
8.5 \\
8.4 \\
8.9\end{array}$ & $\begin{array}{l}.5 \\
.5 \\
.6 \\
.5 \\
.6\end{array}$ & $\begin{array}{l}-2.5 \\
-2.5 \\
-2.4 \\
-2.4 \\
-2.5\end{array}$ & $\begin{array}{l}-3.2 \\
-3.3 \\
-3.3 \\
-3.2 \\
-3.1\end{array}$ & $\begin{array}{l}-2.4 \\
-2.4 \\
-2.5 \\
-2.6 \\
-2.7\end{array}$ & $\begin{array}{l}-1.4 \\
-1.4 \\
-1.4 \\
-1.4 \\
-1.4\end{array}$ & $\begin{array}{l}-.9 \\
-.8 \\
-.8 \\
-.7 \\
-.7\end{array}$ & $\begin{array}{l}-.2 \\
-.2 \\
-.2 \\
-.2 \\
-.2\end{array}$ & $\begin{array}{l}12.9 \\
13.2 \\
13.5 \\
14.3 \\
13.9\end{array}$ & $\begin{array}{r}9.5 \\
10.0 \\
12.0 \\
13.6 \\
13.5\end{array}$ & $\begin{array}{l}15.1 \\
16.0 \\
15.8 \\
15.7 \\
15.0\end{array}$ & $\begin{array}{r}9.3 \\
10.2 \\
10.7 \\
6.3 \\
6.2\end{array}$ \\
\hline $\begin{array}{r}6 \\
7 \\
8 \\
9 \\
10\end{array}$ & $\begin{array}{l}9.3 \\
9.2 \\
8.9 \\
8.5 \\
8.5\end{array}$ & $\begin{array}{l}.6 \\
.6 \\
.6 \\
.6 \\
.5\end{array}$ & $\begin{array}{l}-2.6 \\
-2.4 \\
-2.2 \\
-2.3 \\
-2.4\end{array}$ & $\begin{array}{l}-3.0 \\
-2.8 \\
-2.7 \\
-2.6 \\
-2.5\end{array}$ & $\begin{array}{l}-2.8 \\
-2.7 \\
-2.5 \\
-2.4 \\
-2.3\end{array}$ & $\begin{array}{l}-1.4 \\
-1.3 \\
-1.3 \\
-1.3 \\
-1.3\end{array}$ & $\begin{array}{l}-.6 \\
-.6 \\
-.5 \\
-.5 \\
-.5\end{array}$ & $\begin{array}{l}-.2 \\
-.2 \\
-.2 \\
-.2 \\
-.2\end{array}$ & $\begin{array}{l}13.0 \\
11.4 \\
11.6 \\
12.6 \\
13.7\end{array}$ & $\begin{array}{l}12.5 \\
13.1 \\
14.8 \\
14.8 \\
14.7\end{array}$ & $\begin{array}{l}15.4 \\
16.9 \\
17.8 \\
18.3 \\
17.3\end{array}$ & $\begin{array}{r}7.8 \\
9.0 \\
10.4 \\
12.0 \\
12.5\end{array}$ \\
\hline $\begin{array}{l}11 \\
12 \\
13 \\
14 \\
15\end{array}$ & $\begin{array}{l}8.2 \\
8.8 \\
8.1 \\
7.9 \\
7.9\end{array}$ & $\begin{array}{l}.5 \\
.4 \\
.3 \\
.2 \\
.1\end{array}$ & $\begin{array}{l}-2.5 \\
-2.7 \\
-2.8 \\
-3.0 \\
-2.9\end{array}$ & $\begin{array}{l}-2.4 \\
-2.3 \\
-2.2 \\
-2.2 \\
-2.1\end{array}$ & $\begin{array}{l}-2.2 \\
-2.2 \\
-2.1 \\
-2.0 \\
-2.0\end{array}$ & $\begin{array}{l}-1.3 \\
-1.3 \\
-1.2 \\
-1.2 \\
-1.1\end{array}$ & $\begin{array}{l}-.5 \\
-.5 \\
-.4 \\
-.4 \\
-.4\end{array}$ & $\begin{array}{l}2.8 \\
4.0 \\
6.2 \\
7.3 \\
8.2\end{array}$ & $\begin{array}{r}14.1 \\
11.9 \\
9.0 \\
6.9 \\
4.2\end{array}$ & $\begin{array}{l}13.1 \\
12.5 \\
11.9 \\
10.8 \\
10.8\end{array}$ & $\begin{array}{l}16.1 \\
14.8 \\
13.5 \\
13.0 \\
12.9\end{array}$ & $\begin{array}{l}13.1 \\
12.1 \\
11.6 \\
11.9 \\
12.0\end{array}$ \\
\hline $\begin{array}{l}16 \\
17 \\
18 \\
19 \\
20\end{array}$ & $\begin{array}{l}7.7 \\
7.2 \\
7.0 \\
6.3 \\
5.2\end{array}$ & $\begin{array}{l}-.2 \\
-.4 \\
-.5 \\
-.7 \\
-.7\end{array}$ & $\begin{array}{l}-2.9 \\
-3.0 \\
-2.8 \\
-2.8 \\
-2.7\end{array}$ & $\begin{array}{l}-2.1 \\
-2.0 \\
-2.0 \\
-2.0 \\
-2.0\end{array}$ & $\begin{array}{l}-2.0 \\
-1.9 \\
-1.9 \\
-1.9 \\
-1.8\end{array}$ & $\begin{array}{l}-1.1 \\
-1.1 \\
-1.0 \\
-1.0 \\
-1.0\end{array}$ & $\begin{array}{l}-.4 \\
-.4 \\
-.4 \\
-.4 \\
-.4\end{array}$ & $\begin{array}{r}8.4 \\
9.4 \\
10.0 \\
9.9 \\
8.1\end{array}$ & $\begin{array}{l}3.0 \\
2.5 \\
3.1 \\
5.0 \\
6.5\end{array}$ & $\begin{array}{l}11.2 \\
12.7 \\
12.3 \\
14.2 \\
15.0\end{array}$ & $\begin{array}{l}13.5 \\
14.1 \\
14.7 \\
16.6 \\
17.0\end{array}$ & $\begin{array}{r}\mathrm{e} 11.6 \\
\mathrm{e} 11.3 \\
\mathrm{e} 9.8 \\
\mathrm{e} 8.6 \\
\mathrm{e} 9.2\end{array}$ \\
\hline $\begin{array}{l}21 \\
22 \\
23 \\
24 \\
25\end{array}$ & $\begin{array}{r}4.5 \\
4.6 \\
3.8 \\
1.6 \\
.9\end{array}$ & $\begin{array}{r}-.5 \\
-1.4 \\
-2.3 \\
-2.2 \\
-2.1\end{array}$ & $\begin{array}{l}-3.1 \\
-3.4 \\
-3.5 \\
-3.5 \\
-3.7\end{array}$ & $\begin{array}{l}-2.1 \\
-2.2 \\
-2.3 \\
-2.4 \\
-2.4\end{array}$ & $\begin{array}{l}-1.8 \\
-1.8 \\
-1.7 \\
-1.7 \\
-1.6\end{array}$ & $\begin{array}{l}-1.1 \\
-1.1 \\
-1.1 \\
-1.1 \\
-1.0\end{array}$ & $\begin{array}{l}-.3 \\
-.3 \\
-.3 \\
-.3 \\
-.3\end{array}$ & $\begin{array}{r}8.1 \\
7.9 \\
8.3 \\
10.6 \\
11.0\end{array}$ & $\begin{array}{r}7.7 \\
9.6 \\
10.6 \\
10.7 \\
9.7\end{array}$ & $\begin{array}{l}15.2 \\
15.8 \\
15.6 \\
16.0 \\
15.3\end{array}$ & $\begin{array}{l}16.3 \\
14.5 \\
13.5 \\
13.6 \\
13.9\end{array}$ & $\begin{array}{r}\text { e9.8 } \\
\text { e10.9 } \\
\text { e11.5 } \\
\text { e10.8 } \\
\text { e9.3 }\end{array}$ \\
\hline $\begin{array}{l}26 \\
27 \\
28 \\
29 \\
30 \\
31\end{array}$ & $\begin{array}{r}.9 \\
1.1 \\
.6 \\
.4 \\
.5 \\
.5\end{array}$ & $\begin{array}{r}-2.1 \\
-2.2 \\
-2.3 \\
-2.2 \\
-2.3 \\
---\end{array}$ & $\begin{array}{l}-3.7 \\
-3.5 \\
-3.5 \\
-3.5 \\
-3.3 \\
-3.2\end{array}$ & $\begin{array}{l}-2.4 \\
-2.3 \\
-2.3 \\
-2.3 \\
-2.3 \\
-2.4\end{array}$ & $\begin{array}{r}-1.6 \\
-1.6 \\
-1.5 \\
-1.5 \\
--- \\
---\end{array}$ & $\begin{array}{r}-1.0 \\
-1.0 \\
-1.0 \\
-1.0 \\
-.9 \\
-.9\end{array}$ & $\begin{array}{l}-.3 \\
-.3 \\
-.3 \\
-.3 \\
-.2 \\
-.--\end{array}$ & $\begin{array}{l}11.9 \\
11.8 \\
11.4 \\
10.7 \\
10.7 \\
11.9\end{array}$ & $\begin{array}{r}10.4 \\
11.6 \\
12.3 \\
9.9 \\
9.5 \\
-.-\end{array}$ & $\begin{array}{l}15.2 \\
15.1 \\
15.4 \\
15.6 \\
16.5 \\
15.3\end{array}$ & $\begin{array}{r}14.3 \\
14.9 \\
15.5 \\
13.8 \\
10.1 \\
9.7\end{array}$ & $\begin{array}{r}\mathrm{e} 8.6 \\
\mathrm{e} 8.6 \\
\mathrm{e} 8.7 \\
\mathrm{e} 9.4 \\
\mathrm{e} 10.4 \\
-\end{array}$ \\
\hline $\begin{array}{l}\text { MEAN } \\
\text { MAX } \\
\text { MIN } \\
\text { MED }\end{array}$ & $\begin{array}{r}5.8 \\
9.3 \\
.4 \\
7.7\end{array}$ & $\begin{array}{r}-.5 \\
.6 \\
-2.3 \\
.0\end{array}$ & $\begin{array}{l}-2.9 \\
-2.2 \\
-3.7 \\
-2.8\end{array}$ & $\begin{array}{l}-2.5 \\
-2.0 \\
-3.3 \\
-2.3\end{array}$ & $\begin{array}{l}-2.1 \\
-1.5 \\
-2.8 \\
-2.0\end{array}$ & $\begin{array}{r}-1.2 \\
-.9 \\
-1.4 \\
-1.1\end{array}$ & $\begin{array}{l}-.5 \\
-.2 \\
-.9 \\
-.4\end{array}$ & $\begin{array}{r}6.0 \\
11.9 \\
-.2 \\
8.1\end{array}$ & $\begin{array}{r}9.9 \\
14.3 \\
2.5 \\
10.6\end{array}$ & $\begin{array}{r}13.7 \\
16.5 \\
9.5 \\
14.2\end{array}$ & $\begin{array}{r}14.8 \\
18.3 \\
9.7 \\
14.9\end{array}$ & $\begin{array}{r}10.1 \\
13.1 \\
6.2 \\
10.3\end{array}$ \\
\hline
\end{tabular}

CAL YR 1991 MEAN 2.4 MAX 18.1 MIN -9.6 MED -.1

WTR YR 1992 MEAN 4.3 MAX 18.3 MIN -3.7 MED $\quad .5$

e Estimated 
EAST STEWART BASIN

VEG SPRING NEAR IONE, NV

DAILY TOTAL SOLAR RADIATION, INCIDENTAL, WATER YEAR OCTOBER 1991 TO SEPTEMBER 1992 CALORIES PER SQUARE CENTIMETER

\begin{tabular}{|c|c|c|c|c|c|c|c|c|c|c|c|c|}
\hline DAY & OCT & NOV & DEC & JAN & FEB & MAR & APR & MAY & JUN & JUL & AUG & SEP \\
\hline 1 & 355 & 206 & 131 & 129 & 157 & 307 & 528 & 405 & 506 & 347 & 401 & 240 \\
\hline 2 & 343 & 192 & 134 & 133 & 200 & 157 & 546 & 363 & 439 & 607 & 433 & 364 \\
\hline 3 & 335 & 205 & 130 & 132 & 203 & 178 & 555 & 367 & 555 & 564 & 494 & 300 \\
\hline 4 & 334 & 195 & 132 & 155 & 205 & 315 & 439 & 508 & 632 & 642 & 343 & 309 \\
\hline 5 & 322 & 195 & 130 & 46 & 122 & 329 & 550 & 425 & 525 & 543 & 199 & 484 \\
\hline 6 & 274 & 196 & 136 & 56 & 113 & 314 & 548 & 339 & 289 & 437 & 368 & 519 \\
\hline 7 & 253 & 194 & 61 & 98 & 204 & 271 & 559 & 431 & 328 & 621 & 584 & 506 \\
\hline 8 & 312 & 141 & 55 & 139 & 192 & 199 & 519 & 447 & 371 & 544 & 591 & 488 \\
\hline 9 & 250 & 67 & 123 & 137 & 174 & 307 & 496 & 622 & 541 & 604 & 596 & 494 \\
\hline 10 & 281 & 160 & 129 & 139 & 204 & 380 & 381 & 626 & 605 & 451 & 276 & 428 \\
\hline 11 & 198 & 199 & 126 & 96 & 209 & 385 & 419 & 627 & 633 & 238 & 261 & 387 \\
\hline 12 & 236 & 146 & 124 & 137 & $149^{\circ}$ & 396 & 366 & 399 & 319 & 421 & 129 & 477 \\
\hline 13 & 261 & 125 & 123 & 135 & 203 & 405 & 396 & 549 & 556 & 277 & 286 & 452 \\
\hline 14 & 257 & 91 & 133 & 141 & 252 & 339 & 267 & 550 & 73 & 269 & 237 & 461 \\
\hline 15 & 260 & 106 & 102 & 147 & 156 & 388 & 310 & 579 & 239 & 221 & 256 & 456 \\
\hline 16 & 254 & 160 & 125 & 131 & 191 & 247 & 464 & 451 & 357 & 426 & 314 & e 432 \\
\hline 17 & 250 & 62 & 137 & 143 & 259 & 249 & 322 & 620 & 337 & 324 & 273 & e309 \\
\hline 18 & 250 & 123 & 129 & 149 & 204 & 442 & 600 & 568 & 472 & 357 & 325 & e76 \\
\hline 19 & 239 & 167 & 117 & 150 & 202 & 418 & 591 & 525 & 247 & 623 & 532 & e 429 \\
\hline 20 & 244 & 77 & 124 & 152 & 247 & 308 & 501 & 338 & 390 & 636 & 534 & e 422 \\
\hline 21 & 242 & 156 & 125 & 152 & 183 & 275 & 328 & 489 & 511 & 634 & 501 & e425 \\
\hline 22 & 155 & 143 & 122 & 156 & 271 & 253 & 563 & 451 & 588 & 603 & 512 & e414 \\
\hline 23 & 188 & 138 & 117 & 168 & 280 & 267 & 558 & 481 & 360 & 621 & 540 & e346 \\
\hline 24 & 146 & 134 & 121 & 126 & 287 & 390 & 567 & 592 & 487 & 613 & 460 & e409 \\
\hline 25 & 113 & 148 & 125 & 168 & 288 & 301 & 606 & 404 & 215 & 384 & 515 & e 410 \\
\hline 26 & 81 & 144 & 126 & 141 & 290 & 365 & 449 & 586 & 393 & 410 & 540 & e405 \\
\hline 27 & 113 & 81 & 128 & 171 & 295 & 441 & 610 & 574 & 583 & 299 & 540 & e399 \\
\hline 28 & 237 & 147 & 131 & 81 & 289 & 380 & 605 & 309 & 506 & 375 & 494 & e396 \\
\hline 29 & 91 & 117 & 107 & 182 & 264 & 466 & 594 & 363 & 180 & 383 & 239 & e385 \\
\hline 30 & 241 & 135 & 126 & 191 & --- & 356 & 481 & 391 & 603 & 499 & 294 & e378 \\
\hline 31 & 219 & --- & 127 & 191 & --- & 199 & --- & 560 & --- & 245 & 328 & --- \\
\hline TOTAL & 7334 & 4350 & 3756 & 4272 & 6293 & 10027 & 14718 & $\cdot 14939$ & 12840 & 14218 & 12395 & 12000 \\
\hline CAL YR & 1991 & TOTAL & 120942 & & & & & & & & & \\
\hline WTR YR & 1992 & TOTAL & 117142 & & & & & & & & & \\
\hline
\end{tabular}

e Estimated 
EAST STEWART BASIN

VEG SPRING NEAR IONE, NV

DAILY MEAN RELATIVE HUMIDITY, WATER YEAR OCTOBER 1991 TO SEPTEMBER 1992 PERCENT

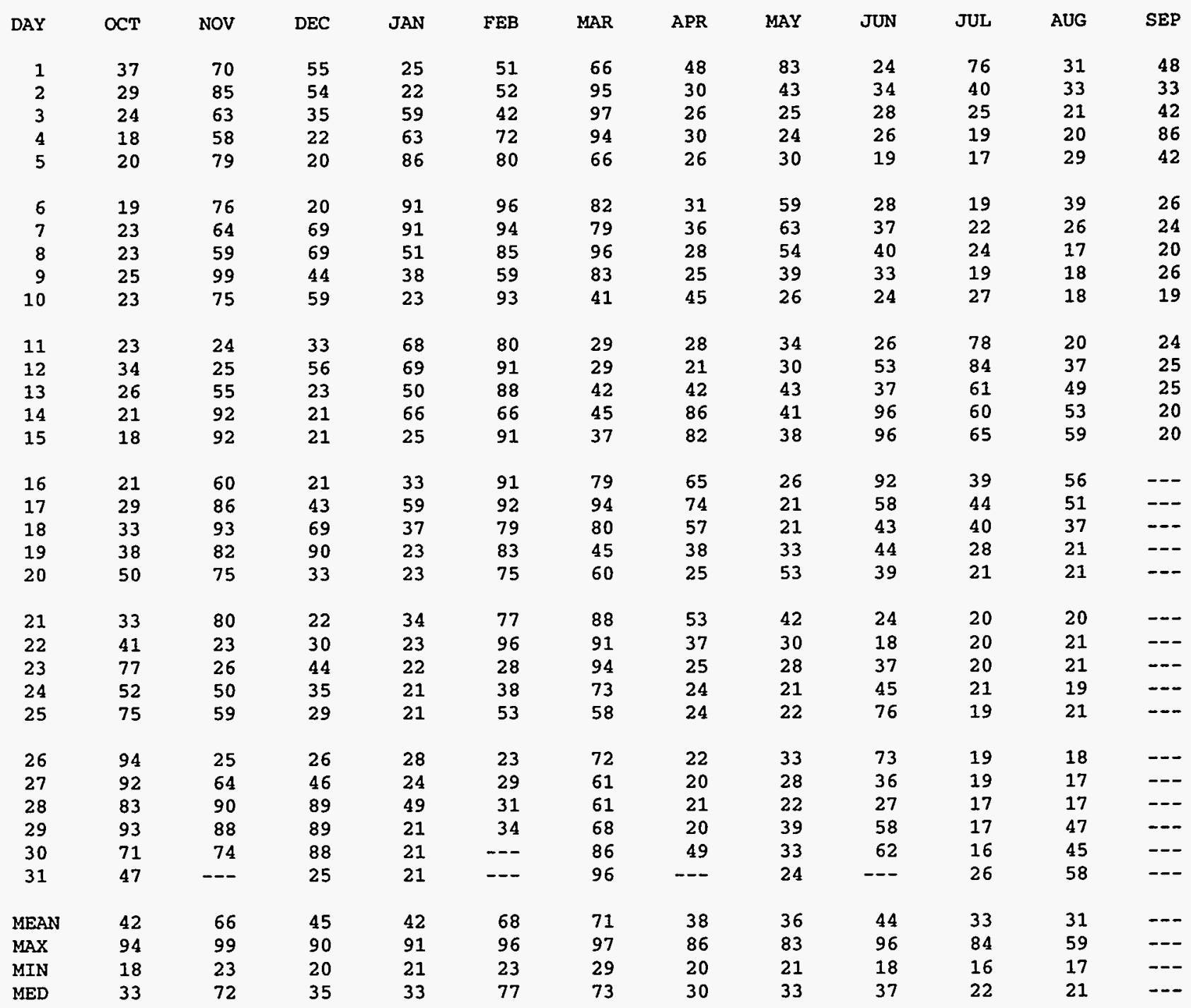

CAL YR 1991 MEAN 49 MAX 100 MIN 15 MED 43 
EAST STEWART BASIN

VEG SPRING NEAR IONE, NV

DAILY TOTAL PRECIPITATION, WATER YEAR OCTOBER 1991 TO SEPTEMBER 1992 INCHES

\begin{tabular}{|c|c|c|c|c|c|c|c|c|c|c|c|c|}
\hline DAY & OCT & NOV & DEC & JAN & FEB & MAR & APR & MAY & JUN & JUL & AUG & SEP \\
\hline 1 & .00 & .00 & .00 & .00 & .00 & .37 & .00 & .00 & .00 & .05 & .00 & .10 \\
\hline 2 & .00 & .00 & .00 & .00 & .00 & .50 & .00 & .00 & .00 & .00 & .00 & .10 \\
\hline 4 & .00 & .00 & .00 & .00 & .00 & .00 & .00 & .00 & .00 & .00 & .00 & .15 \\
\hline 5 & .00 & .00 & .00 & 1.45 & .00 & .00 & .00 & .00 & .00 & .00 & .00 & .15 \\
\hline 6 & .00 & .00 & .10 & .30 & .50 & .55 & .00 & .10 & .00 & .00 & .00 & .00 \\
\hline 7 & .00 & .00 & .10 & .00 & .27 & .05 & .00 & .00 & .00 & .00 & .00 & .00 \\
\hline 8 & .00 & .00 & .00 & .05 & .00 & .00 & .00 & .00 & .00 & .00 & .00 & .00 \\
\hline 9 & .05 & .50 & .00 & .00 & .48 & .00 & .00 & .00 & .00 & .00 & .00 & .00 \\
\hline 10 & .00 & .00 & .00 & .20 & .30 & .00 & .00 & .00 & .00 & .00 & .00 & .05 \\
\hline 11 & .00 & .00 & .00 & .20 & .40 & .00 & .00 & .00 & .00 & e.18 & .00 & .00 \\
\hline 12 & .00 & .00 & .00 & .00 & .40 & .00 & .00 & .00 & e. 07 & .00 & .20 & .00 \\
\hline 13 & .00 & .45 & .00 & .00 & .00 & .00 & .05 & .00 & $e .06$ & .00 & .10 & .00 \\
\hline 15 & .00 & .00 & .00 & .00 & .70 & .00 & .00 & .00 & e. 42 & .27 & .00 & .00 \\
\hline 16 & .05 & .00 & .00 & .00 & .25 & .05 & .00 & .00 & e.15 & .00 & .20 & .00 \\
\hline 17 & .05 & .50 & .00 & .00 & .00 & .22 & .05 & .00 & .00 & .05 & .00 & .15 \\
\hline 18 & .00 & .10 & .05 & .00 & .25 & .00 & .00 & .00 & .00 & .00 & .00 & .00 \\
\hline 19 & .00 & .00 & .05 & .00 & .00 & .00 & .00 & .00 & .00 & .00 & .00 & .00 \\
\hline 20 & .00 & .08 & .00 & .00 & .00 & .00 & .00 & .00 & .00 & .00 & .00 & .00 \\
\hline 21 & .10 & .00 & .00 & .00 & .10 & .10 & .00 & .00 & .00 & .00 & .00 & .00 \\
\hline 22 & .10 & .00 & .00 & .00 & .05 & .50 & .00 & .00 & .00 & .00 & .00 & .00 \\
\hline 23 & .00 & .00 & .00 & .00 & .00 & .00 & .00 & .00 & .00 & .00 & .00 & .00 \\
\hline 24 & .00 & .00 & .00 & .00 & .00 & .00 & .00 & .00 & .00 & .00 & .00 & .00 \\
\hline 25 & .20 & .00 & .00 & .00 & .00 & .00 & .00 & .00 & e. 12 & .00 & .00 & .00 \\
\hline 26 & .40 & .00 & .00 & .00 & .00 & .00 & .00 & .00 & .00 & .00 & .00 & .00 \\
\hline 27 & .00 & .22 & .85 & .00 & .00 & .00 & .00 & .00 & .00 & .00 & .00 & .00 \\
\hline 30 & .00 & .00 & .00 & .00 & --- & .20 & .10 & .00 & e. 08 & .00 & .00 & .00 \\
\hline 31 & .00 & --- & .00 & .00 & --- & .15 & --- & .00 & --- & .05 & .10 & --- \\
\hline TOTAL & 1.55 & 2.00 & 1.70 & 2.25 & 3.80 & 2.94 & 0.30 & 0.10 & 1.31 & 0.70 & 0.75 & 1.75 \\
\hline
\end{tabular}

CAL YR 1991 TOTAL 22.34

WTR YR 1992 TOTAL 19.15

e Estimated 
EAST STEWART CREEK BASIN

VEG SPRING NEAR IONE, NV

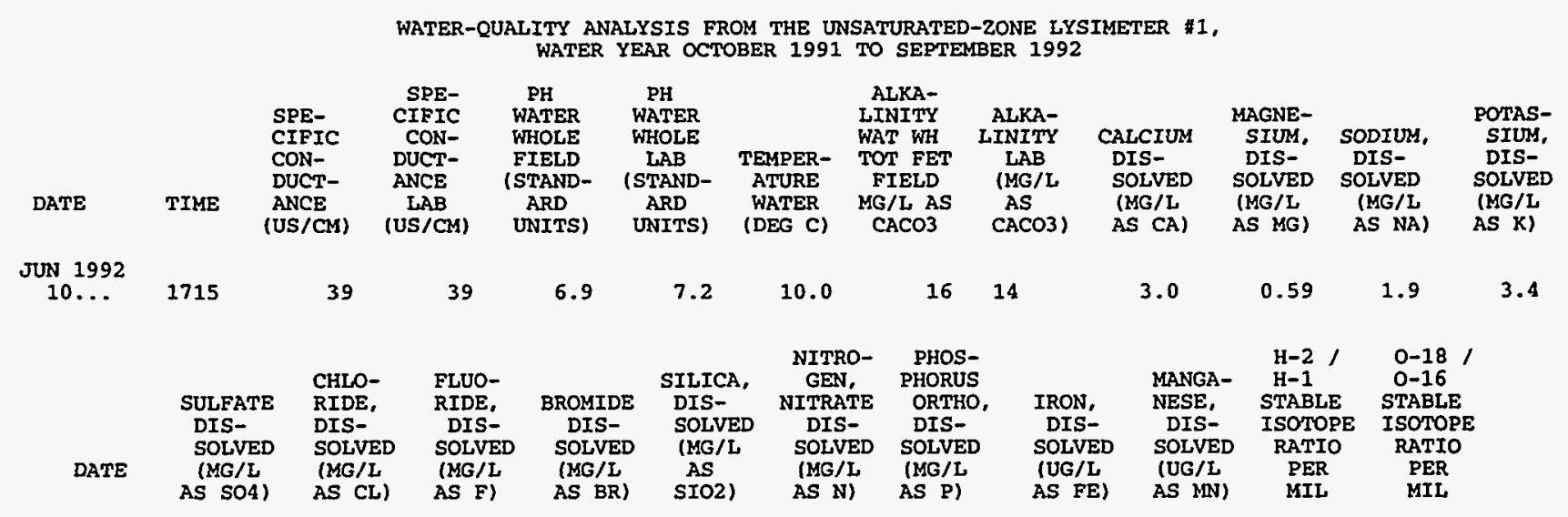

JUN 1992

10 ...

0.72

0.46

$0.08<0.010$

6.6

0.090

0.030

12

$<1 \quad-112.0 \quad-15.20$ 
EAST STEWART CREEK BASIN

VEG SPRING NEAR IONE, NV

SURFACE-WATER QUALITY, WATER YEAR OCTOBER 1991 TO SEPTEMBER 1992

\begin{tabular}{|c|c|c|c|c|c|c|c|c|c|c|c|c|}
\hline ATE & TIME & $\begin{array}{l}\text { SPE- } \\
\text { CIFIC } \\
\text { CON- } \\
\text { DUCT- } \\
\text { ANCE } \\
\text { (US/CM) }\end{array}$ & $\begin{array}{c}\text { SPE- } \\
\text { CIFIC } \\
\text { CON- } \\
\text { DUCT- } \\
\text { ANCE } \\
\text { LAB } \\
\text { (US/CM) }\end{array}$ & $\begin{array}{c}\text { PH } \\
\text { WATER } \\
\text { WHOLE } \\
\text { FIELD } \\
\text { (STAND- } \\
\text { ARD } \\
\text { UNITS) }\end{array}$ & $\begin{array}{c}\text { PH } \\
\text { WATER } \\
\text { WHOLE } \\
\text { LAB } \\
\text { (STAND- } \\
\text { ARD } \\
\text { UNITS) }\end{array}$ & $\begin{array}{c}\text { TEMPER- } \\
\text { ATURE } \\
\text { WATER } \\
\text { (DEG C) }\end{array}$ & $\begin{array}{l}\text { ALKA- } \\
\text { LINITY } \\
\text { WAT WH } \\
\text { TOT FET } \\
\text { FIELD } \\
\text { HG/L AS } \\
\text { CACO3 }\end{array}$ & $\begin{array}{c}\text { ALKA- } \\
\text { LINITY } \\
\text { LAB } \\
\text { (MG/L } \\
\text { AS } \\
\text { CACO3) }\end{array}$ & $\begin{array}{l}\text { CALCIUM } \\
\text { DIS- } \\
\text { SOLVED } \\
\text { (MG/L } \\
\text { AS CA) }\end{array}$ & $\begin{array}{l}\text { MAGNE- } \\
\text { SIUM, } \\
\text { DIS- } \\
\text { SOLVED } \\
\text { (MG/L } \\
\text { AS MG) }\end{array}$ & $\begin{array}{c}\text { SODIUM, } \\
\text { DIS- } \\
\text { SOLVED } \\
\text { (MG/L } \\
\text { AS NA) }\end{array}$ & $\begin{array}{l}\text { POTAS- } \\
\text { SIUM, } \\
\text { DIS- } \\
\text { SOLVED } \\
\text { (MG/L } \\
\text { AS K) }\end{array}$ \\
\hline
\end{tabular}

\begin{tabular}{|c|c|c|c|c|c|c|c|c|c|c|c|c|}
\hline JuL $11 .$. & 1315 & 30 & 30 & 6.3 & 6.6 & 5.0 & 12 & 12 & 2.3 & 0.31 & 3.1 & 0.50 \\
\hline $\operatorname{seP}^{29}$ & 1000 & 28 & 30 & 6.6 & 7.3 & 4.5 & 12 & 9.1 & 2.2 & 0.27 & 3.2 & 0.40 \\
\hline $16 \ldots$ & 1220 & 30 & 30 & 6.6 & 7.3 & 4.5 & 12 & 11 & 2.3 & 0.28 & 3.3 & 0.50 \\
\hline
\end{tabular}

\begin{tabular}{|c|c|c|c|c|c|c|}
\hline & $\begin{array}{l}\text { SULFATE } \\
\text { DIS- } \\
\text { SOLVED }\end{array}$ & $\begin{array}{l}\text { CHLO- } \\
\text { RIDE, } \\
\text { DIS- } \\
\text { SOLVED }\end{array}$ & $\begin{array}{l}\text { FLUO- } \\
\text { RIDE, } \\
\text { DIS- } \\
\text { SOLVED }\end{array}$ & $\begin{array}{c}\text { BROMIDE } \\
\text { DIS- } \\
\text { SOLVED }\end{array}$ & $\begin{array}{l}\text { SILICA, } \\
\text { DIS- } \\
\text { SOLVED } \\
\text { (MG/L }\end{array}$ & $\begin{array}{cc}\text { GEN, } & \text { PHORUS } \\
\text { NITRATE } & \text { ORTHO, } \\
\text { DIS- } & \text { DIS- } \\
\text { SOLVED } & \text { SOLVED }\end{array}$ \\
\hline $\mathrm{Am}$ & & ir & (MG/L & (MG/L & & \\
\hline
\end{tabular}
$\begin{array}{llllll}\text { (MG/L } & \text { (MG/L } & \text { (MG/L } & \text { (MG/L } & \text { AS } & \text { SOLVED SOLVED } \\ \text { (MG/L } & \text { (MG/T }\end{array}$

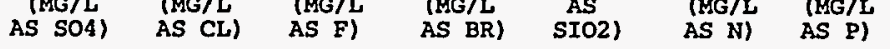

$\begin{array}{llll} & & \text { H-2 / } & 0-18 / \\ \text { IRON, } & \text { MENGA- } & \text { H-1 } & 0-16 \\ \text { DIS- } & \text { DIS- } & \text { STABLE } & \text { STABLE } \\ \text { SOLVED } & \text { SOLVED } & \text { RATIO } & \text { ISOTOPE } \\ \text { (UG/L } & \text { (UG/L } & \text { PATIO } \\ \text { AS FE) } & \text { AS MN) } & \text { MIL } & \text { PER } \\ \text { MIL } & \text { MIL }\end{array}$

\begin{tabular}{|c|c|c|c|c|c|c|c|c|c|c|c|}
\hline $\begin{array}{c}\text { JUN } 1992 \\
11 \ldots \\
\text { JUL }\end{array}$ & 1.2 & 0.55 & 0.03 & $<0.010$ & 18 & 0.290 & $<0.010$ & $<3$ & $<1$ & -121.0 & -16.45 \\
\hline $\begin{array}{l}29 \\
\text { SEP }\end{array}$ & 1.1 & 0.56 & 0.07 & $<0.010$ & 17 & 0.150 & $<0.010$ & 3 & $<1$ & -122.0 & -16.60 \\
\hline $16 \ldots$ & 1.2 & 0.59 & 0.05 & $<0.010$ & 19 & 0.220 & $<0.010$ & $<3$ & $<1$ & $-121 \cdot 0$ & -16.65 \\
\hline
\end{tabular}




\section{EAST STEWART BASIN}

EAST STEWART CREEK NEAR IONE, NV

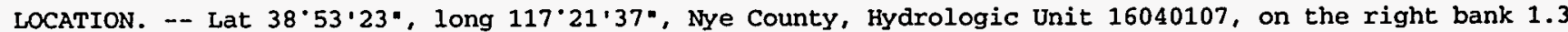
$\mathrm{mi}$ upstream from the Columbine campground, and $13.3 \mathrm{mi}$ east of Ione on state Route 21 .

DRAINAGE AREA. -- $0.36 \mathrm{mi}^{2}$.

PERIOD OF RECORD. -- September 1984 to current year.

GAGE. - - Two water-stage recorders on a 9 in Parshall flume and a standard 90 degree v-notch weir, and a crest-stage gage. Elevation of gage is $9,455 \mathrm{ft}$ above sea level, from topographic map. The fiume is considered the primary gage. See REMARKS for more information.

REMARKS. - The weather equipment is located approximately $150 \mathrm{ft}$ northeast of the surface-water equipment. The surface-water-quality samples are taken upstream from the weir pool and the unsaturated-zone waterquality samples are taken approximately $100 \mathrm{ft}$ upstream on the left bank from the trail crossing or about $700 \mathrm{ft}$ upstream from the surface water site. Discharge records are determined from the 9 in Parshall flume. Accuracy of measurement by the flume diminishes below a discharge of $0.25 \mathrm{ft} / \mathrm{s}$. Daily discharges below $0.25 \mathrm{ft}^{3} / \mathrm{s}$ are considered fair; discharges above $0.25 \mathrm{ft}^{3} / \mathrm{s}$ are considered good. Ice effects are considered minimal if they occurred at all during the winter months. Estimated periods of daily record are considered poor. Values for the daily maximum and minimum air temperature were obtained from the forty minute average values.

PERIOD OF DAILY RECORD. --

AIR TEMPERATURE: January 1986 to current year.

SOIL TEMPERATURE: January 1986 to current year.

SOLAR RADIATION: January 1986 to current year.

RELATIVE HUMIDITY: October 1990 to current year.

TOTAL PRECIPITATION: October 1985 to current year.

DISCHARGE: May 1987 to current year.

EXTREMES FOR PERIOD OF RECORD. --

AIR TEMPERATURE: Maximum daily, $25.6^{\circ} \mathrm{C}$, July 4, 1991; minimum daily, -28.2 ${ }^{\circ}$, Dec. 22, 1990.

See REMARKS for more information.

SOIL TEMPERATURE: Maximum daily mean, $21.2^{\circ} \mathrm{C}$, July 18 , 1991 ; minimum daily mean, $-5.6^{\circ} \mathrm{C}$,

Dec. 20-21, 1989 .

SOLAR RADIATION: Maximum daily total, $809 \mathrm{cal} / \mathrm{cm}^{2}$, June 29, 1990; minimum daily total.

$7 \mathrm{cal} / \mathrm{cm}^{2}$, Nov. 2, 1987

RELATIVE HUMIDITY: Maximum daily mean, 99.8 pct, June 14, 1992; minimum daily mean, 15.3 pct, Aug. 30 , 1991.

TOTAL PRECIPITATION: Maximum daily, 1.50 in, Jan. 5, 1992; no precipitation, most days of record. DISCHARGE: Maximun daily mean, $3.4 \mathrm{ft}^{3} / \mathrm{s}$, June 13-14, 1991; minimum daily mean, $0.03 \mathrm{ft}^{3} / \mathrm{s}$, Feb. 3-4, 1991.

EXTREMES FOR CURRENT YEAR. --

AIR TEMPERATURE: Maximum daily, $25.3^{\circ} \mathrm{C}$, July 30 ; minimum daily, $-18.2^{\circ} \mathrm{C}$, Nov. 30 . See REMARKS for more information.

SOIL TEMPERATURE: Maximum daily mean, $20.1^{\circ} \mathrm{C}$, Aug. 9; minimum daily mean, $-0.6^{\circ} \mathrm{C}, \mathrm{Feb}^{\circ}$ 5-7. SOLAR RADIATION: Maximum daily total, $732 \mathrm{cal} / \mathrm{cm}^{2}$, July 2; minimum daily total, $18 \mathrm{cal} / \mathrm{cm}^{2}$, Jan. 6.

RELATIVE HUMIDITY: Maximum daily mean, 99.8 pct, June 14; minimum daily mean, 16.1 pet, July 30. TOTAL PRECIPITATION: Maximum daily, 1.50 in, Jan. 5; no precipitation, most days of record. DISCHARGE: Maximum daily mean, $1.6 \mathrm{ft}^{3} / \mathrm{s}$, May 17-20; minimum daily mean, $0.10 \mathrm{ft}^{3} / \mathrm{s}, \mathrm{Mar} .7-12$. 
EAST STEWART BASIN

EAST STEWART CREEK NEAR IONE, NV

DAILY MEAN AIR TEMPERATURE, WATER YEAR OCTOBER 1991 TO SEPTEMBER 1992 DEGREES CELSIUS

\begin{tabular}{|c|c|c|c|c|c|c|c|c|c|c|c|c|}
\hline DAY & OCT & NOV & DEC & JAN & FEB & MAR & APR & MAY & JUN & JUL & AUG & SEP \\
\hline $\begin{array}{l}1 \\
2 \\
3 \\
4 \\
5\end{array}$ & $\begin{array}{l}8.5 \\
9.0 \\
9.0 \\
8.1 \\
9.7\end{array}$ & $\begin{array}{r}-2.2 \\
-.8 \\
1.7 \\
2.3 \\
4.0\end{array}$ & $\begin{array}{r}-7.9 \\
-3.0 \\
-.9 \\
-2.7 \\
-1.0\end{array}$ & $\begin{array}{l}-3.6 \\
-2.2 \\
-1.8 \\
-2.7 \\
-4.8\end{array}$ & $\begin{array}{l}-3.3 \\
-5.4 \\
-6.0 \\
-3.7 \\
-3.3\end{array}$ & $\begin{array}{l}-1.7 \\
-2.7 \\
-2.1 \\
-2.6 \\
-1.3\end{array}$ & $\begin{array}{l}2.6 \\
4.9 \\
5.2 \\
3.2 \\
1.1\end{array}$ & $\begin{array}{r}1.6 \\
6.0 \\
9.1 \\
10.5 \\
10.6\end{array}$ & $\begin{array}{l}13.9 \\
13.5 \\
13.8 \\
13.7 \\
11.1\end{array}$ & $\begin{array}{r}4.0 \\
9.8 \\
13.9 \\
14.0 \\
13.0\end{array}$ & $\begin{array}{l}17.2 \\
17.8 \\
15.9 \\
15.7 \\
14.9\end{array}$ & $\begin{array}{r}8.8 \\
12.0 \\
9.6 \\
3.8 \\
7.7\end{array}$ \\
\hline $\begin{array}{r}6 \\
7 \\
8 \\
9 \\
10\end{array}$ & $\begin{array}{r}11.6 \\
8.5 \\
9.6 \\
9.8 \\
11.4\end{array}$ & $\begin{array}{r}4.0 \\
5.4 \\
6.9 \\
1.6 \\
.9\end{array}$ & $\begin{array}{r}3.4 \\
-3.0 \\
-6.0 \\
-3.7 \\
-3.5\end{array}$ & $\begin{array}{r}-10.0 \\
-9.5 \\
-5.4 \\
.1 \\
2.6\end{array}$ & $\begin{array}{l}-2.8 \\
-1.5 \\
-2.2 \\
-4.3 \\
-3.9\end{array}$ & $\begin{array}{l}-4.6 \\
-4.9 \\
-4.6 \\
-3.0 \\
-1.9\end{array}$ & $\begin{array}{l}.4 \\
2.7 \\
2.8 \\
4.6 \\
4.7\end{array}$ & $\begin{array}{l}8.3 \\
8.3 \\
9.3 \\
3.4 \\
7.0\end{array}$ & $\begin{array}{r}9.8 \\
9.2 \\
10.6 \\
13.0 \\
13.4\end{array}$ & $\begin{array}{l}12.4 \\
11.4 \\
12.8 \\
13.1 \\
13.7\end{array}$ & $\begin{array}{l}14.3 \\
16.7 \\
17.2 \\
16.9 \\
17.3\end{array}$ & $\begin{array}{r}8.4 \\
8.7 \\
9.9 \\
12.5 \\
15.1\end{array}$ \\
\hline $\begin{array}{l}11 \\
12 \\
13 \\
14 \\
15\end{array}$ & $\begin{array}{r}11.5 \\
9.0 \\
9.8 \\
11.9 \\
11.3\end{array}$ & $\begin{array}{r}3.3 \\
4.7 \\
1.4 \\
-8.5 \\
-7.7\end{array}$ & $\begin{array}{r}-4.6 \\
-4.5 \\
-3.7 \\
1.2 \\
4.1\end{array}$ & $\begin{array}{r}-8.9 \\
-10.5 \\
-5.4 \\
-5.1 \\
-1.3\end{array}$ & $\begin{array}{l}-4.1 \\
-3.4 \\
-4.9 \\
-4.5 \\
-7.2\end{array}$ & $\begin{array}{r}1.1 \\
1.4 \\
2.4 \\
.9 \\
-.2\end{array}$ & $\begin{array}{r}7.4 \\
6.2 \\
4.9 \\
1.9 \\
.9\end{array}$ & $\begin{array}{r}8.7 \\
8.7 \\
10.1 \\
9.6 \\
8.6\end{array}$ & $\begin{array}{r}12.8 \\
3.1 \\
.4 \\
-3.5 \\
-2.0\end{array}$ & $\begin{array}{r}9.6 \\
8.5 \\
9.9 \\
11.7 \\
11.6\end{array}$ & $\begin{array}{l}18.4 \\
16.0 \\
16.0 \\
16.0 \\
14.8\end{array}$ & $\begin{array}{r}13.1 \\
7.9 \\
8.7 \\
11.0 \\
10.5\end{array}$ \\
\hline $\begin{array}{l}16 \\
17 \\
18 \\
19 \\
20\end{array}$ & $\begin{array}{r}10.4 \\
8.7 \\
7.5 \\
6.9 \\
3.9\end{array}$ & $\begin{array}{r}-1.6 \\
-1.4 \\
-8.2 \\
-1.0 \\
2.9\end{array}$ & $\begin{array}{r}1.2 \\
1.0 \\
-2.5 \\
-11.1 \\
-4.7\end{array}$ & $\begin{array}{l}-1.5 \\
-4.8 \\
-4.7 \\
-2.0 \\
-4.4\end{array}$ & $\begin{array}{r}-9.2 \\
-7.5 \\
-3.8 \\
.3 \\
.5\end{array}$ & $\begin{array}{l}-4.1 \\
-6.3 \\
-5.1 \\
-1.6 \\
-1.5\end{array}$ & $\begin{array}{r}3.1 \\
4.0 \\
-2.4 \\
-.3 \\
5.5\end{array}$ & $\begin{array}{r}11.3 \\
12.1 \\
11.2 \\
8.6 \\
3.2\end{array}$ & $\begin{array}{r}2.5 \\
6.8 \\
8.7 \\
9.4 \\
10.8\end{array}$ & $\begin{array}{l}14.9 \\
14.3 \\
13.7 \\
13.1 \\
13.0\end{array}$ & $\begin{array}{l}14.5 \\
16.5 \\
18.1 \\
17.1 \\
14.6\end{array}$ & $\begin{array}{r}12.1 \\
8.8 \\
4.2 \\
8.9 \\
9.7\end{array}$ \\
\hline $\begin{array}{l}21 \\
22 \\
23 \\
24 \\
25\end{array}$ & $\begin{array}{r}5.6 \\
6.1 \\
-2.5 \\
-3.0 \\
-1.1\end{array}$ & $\begin{array}{r}-4.1 \\
-5.8 \\
-1.3 \\
.7 \\
.7\end{array}$ & $\begin{array}{l}-1.7 \\
-2.8 \\
-1.7 \\
-3.0 \\
-.3\end{array}$ & $\begin{array}{r}-5.8 \\
-5.7 \\
-1.5 \\
.8 \\
1.7\end{array}$ & $\begin{array}{r}.6 \\
-2.5 \\
-2.6 \\
1.5 \\
1.2\end{array}$ & $\begin{array}{r}-2.3 \\
-2.6 \\
-3.9 \\
-1.4 \\
.4\end{array}$ & $\begin{array}{r}2.8 \\
.1 \\
3.2 \\
8.4 \\
10.7\end{array}$ & $\begin{array}{r}5.4 \\
7.2 \\
10.3 \\
13.1 \\
12.9\end{array}$ & $\begin{array}{r}13.6 \\
15.8 \\
14.2 \\
12.4 \\
6.7\end{array}$ & $\begin{array}{l}12.8 \\
13.7 \\
10.9 \\
13.4 \\
14.5\end{array}$ & $\begin{array}{r}12.9 \\
7.6 \\
6.4 \\
8.0 \\
9.6\end{array}$ & $\begin{array}{r}12.0 \\
15.8 \\
13.2 \\
9.5 \\
4.9\end{array}$ \\
\hline $\begin{array}{l}26 \\
27 \\
28 \\
29 \\
30 \\
31\end{array}$ & $\begin{array}{r}-2.5 \\
-9.3 \\
-9.8 \\
-7.5 \\
-9.0 \\
-.7\end{array}$ & $\begin{array}{r}2.5 \\
-4.0 \\
-11.0 \\
-13.2 \\
-15.1 \\
---\end{array}$ & $\begin{array}{r}1.5 \\
-.8 \\
-6.3 \\
-6.2 \\
-7.3 \\
-4.5\end{array}$ & $\begin{array}{r}-.5 \\
-. .4 \\
-1.2 \\
.5 \\
4.6 \\
4.7\end{array}$ & $\begin{array}{l}3.1 \\
1.5 \\
2.2 \\
1.4 \\
--- \\
---\end{array}$ & $\begin{array}{r}-.7 \\
.4 \\
.9 \\
.8 \\
.0 \\
.5\end{array}$ & $\begin{array}{r}10.3 \\
9.2 \\
11.0 \\
12.6 \\
6.9 \\
---\end{array}$ & $\begin{array}{r}10.7 \\
9.9 \\
10.9 \\
9.7 \\
10.7 \\
12.3\end{array}$ & $\begin{array}{r}10.5 \\
15.0 \\
14.4 \\
7.1 \\
5.9 \\
-\end{array}$ & $\begin{array}{l}15.5 \\
16.5 \\
17.7 \\
17.4 \\
18.8 \\
16.5\end{array}$ & $\begin{array}{r}10.1 \\
12.0 \\
12.4 \\
9.4 \\
8.6 \\
6.7\end{array}$ & $\begin{array}{r}8.1 \\
9.8 \\
12.1 \\
14.4 \\
13.8 \\
-.-\end{array}$ \\
\hline $\begin{array}{l}\text { MEAN } \\
\text { MAX } \\
\text { MIN } \\
\text { MED }\end{array}$ & $\begin{array}{r}4.9 \\
11.9 \\
-9.8 \\
8.5\end{array}$ & $\begin{array}{r}-1.4 \\
6.9 \\
-15.1 \\
.0\end{array}$ & $\begin{array}{r}-2.7 \\
4.1 \\
-11.1 \\
-3.0\end{array}$ & $\begin{array}{r}-2.9 \\
4.7 \\
-10.5 \\
-2.2\end{array}$ & $\begin{array}{r}-2.5 \\
3.1 \\
-9.2 \\
-3.3\end{array}$ & $\begin{array}{r}-1.6 \\
2.4 \\
-6.3 \\
-1.6\end{array}$ & $\begin{array}{r}4.6 \\
12.6 \\
-2.4 \\
4.3\end{array}$ & $\begin{array}{r}9.0 \\
13.1 \\
1.6 \\
9.6\end{array}$ & $\begin{array}{r}9.6 \\
15.8 \\
-3.5 \\
10.7\end{array}$ & $\begin{array}{r}13.1 \\
18.8 \\
4.0 \\
13.1\end{array}$ & $\begin{array}{r}13.9 \\
18.4 \\
6.4 \\
14.9\end{array}$ & $\begin{array}{r}10.2 \\
15.8 \\
3.8 \\
9.7\end{array}$ \\
\hline
\end{tabular}

CAL YR 1991 MEAN 3.0 MAX 19.1 MIN -15.1 MED 1.4

WTR YR 1992 MEAN 4.5 MAX 18.8 MIN -15.1 MED 4.4 
EAST STEWART BASIN

EAST STEWART CREEK NEAR IONE, NV

DAILY MAXIMUM AIR TEMPERATURE, WATER YEAR OCTOBER 1991 TO SEPTEMBER 1992 DEGREES CELSIUS

\begin{tabular}{|c|c|c|c|c|c|c|c|c|c|c|c|c|}
\hline DAY & OCT & NOV & DEC & JAN & FEB & MAR & APR & MAY & JUN & JUL & AUG & SEP \\
\hline 1 & 17.7 & 3.0 & -4.0 & 4.4 & -.8 & 3.6 & 8.9 & 6.9 & 21.7 & 10.8 & 24.9 & 15.6 \\
\hline 2 & 17.9 & 4.5 & 1.1 & 3.4 & -1.5 & .3 & 13.0 & 11.6 & 20.1 & 17.2 & 24.1 & 16.9 \\
\hline 3 & 18.0 & 7.7 & 6.9 & 1.2 & -2.7 & 1.2 & 12.4 & 14.6 & 21.0 & 19.3 & 23.3 & 16.1 \\
\hline 4 & 17.5 & 9.8 & 6.0 & .8 & 1.1 & 3.6 & 9.1 & 17.0 & 20.0 & 20.1 & 24.1 & 10.7 \\
\hline 5 & 18.5 & 10.6 & 6.4 & -1.4 & -1.0 & 5.0 & 6.7 & 17.2 & 17.6 & 20.1 & 20.7 & 15.2 \\
\hline 6 & 18.4 & 10.7 & 8.2 & -8.4 & -1.0 & -.5 & 6.5 & 15.1 & 15.0 & 18.7 & 20.0 & 14.1 \\
\hline 7 & 16.3 & 12.8 & 4.1 & -.5 & 3.2 & .7 & 9.7 & 15.5 & 16.1 & 19.5 & 22.1 & $17: 5$ \\
\hline 8 & 16.8 & 9.7 & -2.0 & 1.8 & 2.7 & -2.4 & 11.0 & 14.5 & 17.4 & 19.8 & 22.9 & 18.7 \\
\hline 9 & 16.0 & 2.9 & 5.3 & 7.6 & .6 & 3.0 & 10.8 & 8.7 & 18.8 & 21.5 & 25.3 & 20.7 \\
\hline 10 & 18.1 & 5.4 & 3.4 & 6.0 & -1.3 & 6.4 & 9.6 & 14.0 & 18.6 & 20.9 & 22.9 & 20.1 \\
\hline 11 & 16.5 & 9.9 & .6 & 1.9 & -1.7 & 7.9 & 10.3 & 15.5 & 18.5 & 16.0 & 24.4 & 18.3 \\
\hline 12 & 15.1 & 12.6 & 2.0 & -6.4 & -2.3 & 8.5 & 9.2 & 14.6 & 9.4 & 14.5 & 22.9 & 15.6 \\
\hline 13 & 16.5 & 6.5 & 3.2 & .4 & -.8 & 6.0 & 10.8 & 15.6 & 8.4 & 16.2 & 23.9 & 16.4 \\
\hline 14 & 18.6 & -6.3 & 6.7 & -.6 & .6 & 3.8 & 6.3 & 16.4 & -1.8 & 19.8 & 22.4 & 16.8 \\
\hline 15 & 20.1 & -3.8 & 5.6 & 5.5 & -5.0 & 4.5 & 7.4 & 15.9 & 1.4 & 17.0 & 20.7 & 17.5 \\
\hline 16 & 15.4 & 4.8 & 6.5 & 7.3 & -6.0 & -.1 & 9.8 & 17.4 & 8.4 & 22.4 & 21.5 & 17.7 \\
\hline 17 & 14.7 & 2.2 & 3.1 & .5 & -3.0 & -2.2 & 8.3 & 17.0 & 14.2 & 22.2 & 23.1 & 15.7 \\
\hline 18 & 14.9 & -6.7 & .5 & -1.0 & .4 & 2.5 & 2.4 & 16.0 & 14.9 & 20.2 & 24.0 & 6.0 \\
\hline 19 & 12.8 & 6.6 & -8.6 & 2.6 & 3.5 & 5.2 & 7.3 & 14.7 & 15.1 & 21.0 & 24.5 & 16.8 \\
\hline 20 & 9.4 & 4.9 & 2.7 & 4.8 & 3.7 & 2.9 & 12.5 & 9.7 & 17.3 & 20.5 & 22.6 & 18.3 \\
\hline 21 & 11.7 & .6 & 5.7 & -.9 & 4.3 & 3.6 & 7.6 & 11.3 & 20.4 & 21.5 & 20.3 & 21.0 \\
\hline 22 & 9.4 & 1.9 & 2.8 & 2.4 & .1 & 1.3 & 6.2 & 12.9 & 21.8 & 20.2 & 14.2 & 20.8 \\
\hline 23 & 1.8 & 8.3 & 3.4 & 6.2 & 3.0 & .5 & 11.8 & 15.5 & 20.3 & 18.7 & 15.8 & 18.4 \\
\hline 24 & .1 & 6.3 & 3.5 & 7.5 & 7.5 & 6.3 & 14.6 & 19.2 & 18.4 & 20.7 & 17.0 & 16.1 \\
\hline 25 & 1.3 & 6.2 & 4.9 & 4.8 & 5.4 & 6.7 & 16.3 & 20.0 & 11.8 & 21.1 & 17.6 & 13.5 \\
\hline 26 & 1.3 & 7.4 & 4.4 & 3.5 & 10.0 & 4.3 & 15.2 & 16.4 & 17.4 & 21.5 & 19.5 & 17.3 \\
\hline 27 & -6.2 & 3.8 & 1.9 & 6.2 & 10.9 & 8.0 & 17.8 & 17.2 & 22.4 & 23.4 & 21.4 & 18.7 \\
\hline 28 & -4.7 & -7.4 & -4.0 & 3.1 & 10.1 & 6.7 & 19.2 & 15.1 & 20.2 & 24.6 & 20.8 & 20.5 \\
\hline 29 & -4.4 & -10.0 & -5.1 & 5.3 & 4.4 & 6.8 & 17.5 & 15.2 & 11.7 & 25.0 & 16.9 & 19.0 \\
\hline 30 & -1.9 & -11.8 & -1.7 & 9.6 & --- & 4.0 & 13.3 & 15.8 & 12.7 & 25.3 & 14.6 & 18.2 \\
\hline 31 & 5.8 & --- & 4.2 & 6.7 & --- & 4.2 & --- & 19.8 & --- & 22.5 & 13.9 & $\cdots$ \\
\hline MAX & 20.1 & 12.8 & 8.2 & 9.6 & 10.9 & 8.5 & 19.2 & 20.0 & 22.4 & 25.3 & 25.3 & 21.0 \\
\hline
\end{tabular}

CAL YR 1991 MAX 25.6

WTR YR 1992 MAX 25.3 
EAST STEWART BASIN

EAST STEWART CREEK NEAR IONE, NV

DAILY MINIMUM AIR TEMPERATURE, WATER YEAR OCTOBER 1991 TO SEPTEMBER 1992 DEGREES CELSIUS

\begin{tabular}{|c|c|c|c|c|c|c|c|c|c|c|c|c|}
\hline DAY & OCT & NOV & DEC & JAN & FEB & MAR & APR & MAY & JUN & JUL & AUG & SEP \\
\hline 1 & 2.5 & -5.4 & -13.8 & -7.8 & -8.1 & -3.5 & -1.9 & -2.8 & 5.3 & -1.5 & 8.9 & 2.5 \\
\hline 2 & 3.6 & -4.8 & -5.5 & -6.7 & -8.2 & -4.6 & -.9 & .7 & 6.5 & -.2 & 13.6 & 7.3 \\
\hline 3 & 3.3 & -2.2 & -5.5 & -5.6 & -8.1 & -5.3 & -1.7 & 4.5 & 5.5 & 7.4 & 8.5 & -.1 \\
\hline 4 & 2.0 & -2.4 & -5.8 & -6.7 & -7.6 & -7.2 & -1.8 & 4.5 & 5.4 & 7.1 & 8.5 & -.7 \\
\hline 5 & 3.7 & .5 & -5.3 & -8.5 & -5.1 & -7.4 & -3.3 & 3.4 & 2.8 & 5.1 & 9.5 & .1 \\
\hline 6 & 4.5 & .7 & -1.8 & -10.7 & -4.1 & -8.0 & -3.6 & 3.4 & 3.5 & 5.1 & 10.9 & 2.4 \\
\hline 7 & 3.4 & .1 & -8.5 & -14.4 & -3.3 & -9.8 & -3.2 & 2.3 & 2.6 & 2.3 & 10.4 & 1.5 \\
\hline 8 & 2.5 & 3.3 & -8.9 & -13.5 & -6.4 & -5.9 & -3.0 & 3.2 & 3.1 & 5.9 & 10.8 & 3.2 \\
\hline 9 & 4.0 & .4 & -9.0 & -4.9 & -8.6 & -6.7 & -2.3 & -1.3 & 7.6 & 3.9 & 9.2 & 4.6 \\
\hline 10 & 6.8 & -3.1 & -6.2 & -3.5 & -4.9 & -9.5 & .2 & -1.6 & 9.8 & 6.3 & 10.3 & 11.0 \\
\hline 11 & 7.3 & -.6 & -7.7 & -13.0 & -5.5 & -4.7 & 5.1 & 1.8 & 8.2 & 5.7 & 13.0 & 4.8 \\
\hline 12 & 4.4 & -.3 & -9.5 & -14.9 & -5.1 & -5.2 & 3.7 & 1.4 & -6.1 & 4.6 & 10.5 & 1.8 \\
\hline 13 & 4.9 & -6.8 & -7.7 & -10.9 & -9.2 & .4 & .5 & 4.2 & -7.8 & 3.4 & 11.0 & .7 \\
\hline 14 & 4.6 & -10.8 & -2.2 & -7.7 & -7.9 & -1.7 & -1.8 & 3.5 & -6.0 & 5.3 & 12.2 & 3.3 \\
\hline 15 & 3.7 & -10.1 & 2.5 & -7.9 & -10.2 & -3.6 & -3.4 & 1.4 & -6.6 & 8.1 & 10.5 & 3.1 \\
\hline 16 & 4.1 & -8.5 & -3.0 & -6.1 & -13.5 & -6.8 & -3.5 & 4.8 & -2.5 & 7.1 & 8.1 & 7.0 \\
\hline 17 & 3.4 & -6.9 & -2.2 & -8.0 & -11.0 & -9.9 & -2.9 & 8.2 & -.6 & 8.0 & 11.3 & 3.2 \\
\hline 18 & 3.1 & -11.7 & -9.0 & -8.2 & -7.6 & -11.3 & -6.6 & 6.0 & 1.9 & 7.0 & 12.6 & 2.7 \\
\hline 19 & 2.4 & -8.7 & -13.6 & -6.5 & -1.9 & -8.1 & -6.5 & -.5 & 1.7 & 4.8 & 8.8 & 1.9 \\
\hline 20 & -.8 & -.2 & -10.8 & -9.4 & -3.7 & -3.6 & -2.5 & -2.9 & 5.5 & 4.7 & 7.4 & 3.8 \\
\hline 21 & -2.1 & -11.3 & -7.8 & -9.1 & -3.1 & -6.1 & -3.1 & -.8 & 4.9 & 3.1 & 3.7 & 4.6 \\
\hline 22 & 1.8 & -11.8 & -7.9 & -9.6 & -7.6 & -7.5 & -5.5 & 2.0 & 10.3 & 4.0 & -.3 & 12.6 \\
\hline 23 & -6.5 & -5.7 & -5.8 & -8.1 & -9.7 & -8.4 & -5.2 & 1.8 & 9.1 & 2.3 & -2.8 & 6.2 \\
\hline 24 & -7.0 & -2.9 & -7.4 & -4.3 & -3.8 & -8.6 & 3.8 & 7.1 & 4.3 & 7.4 & .8 & -.1 \\
\hline 25 & -3.6 & -3.5 & -6.7 & -4.1 & -1.9 & -2.1 & 6.7 & 6.0 & 2.0 & 6.9 & 2.1 & -.9 \\
\hline 26 & -6.4 & -2.3 & -.2 & -5.5 & -2.5 & -4.9 & 3.4 & 5.5 & 5.2 & 8.5 & 1.6 & 1.7 \\
\hline 27 & -11.8 & -13.2 & -5.4 & -7.1 & -3.7 & -5.9 & 2.0 & 2.3 & 5.6 & 10.2 & 3.0 & 3.2 \\
\hline 28 & -13.7 & -12.8 & -7.8 & -4.4 & -3.7 & -4.8 & 2.5 & 6.5 & 8.6 & 12.0 & 4.8 & 4.5 \\
\hline 29 & -12.9 & -15.4 & -7.1 & -2.9 & -1.9 & -3.7 & 7.5 & 3.0 & 1.6 & 9.0 & 4.1 & 11.0 \\
\hline 30 & -15.0 & -18.2 & -10.9 & -1.5 & -- & -2.6 & -1.7 & 5.7 & .1 & 13.9 & 2.2 & 11.5 \\
\hline 31 & -6.5 & --- & -10.1 & -.6 & --- & -2.1 & --- & 3.8 & --- & 12.0 & .6 & -- \\
\hline MIN & -15.0 & -18.2 & -13.8 & -14.9 & -13.5 & -11.3 & -6.6 & -2.9 & -7.8 & -1.5 & -2.8 & -.9 \\
\hline
\end{tabular}

CAL YR 1991 MIN -18.2

WTR YR 1992 MIN -18.2 
EAST STEWART BASIN

EAST STEWART CREEK NEAR IONE, NV

DAILY MEAN SOIL TEMPERATURE, WATER YEAR OCTOBER 1991 TO SEPTEMBER 1992 DEGREES CELSIUS

\begin{tabular}{|c|c|c|c|c|c|c|c|c|c|c|c|c|}
\hline DAY & OCT & NOV & DEC & JAN & FEB & MAR & APR & MAY & JUN & JUL & AUG & SEP \\
\hline 1 & 6.3 & 1.2 & .3 & -.1 & -.4 & -.3 & .0 & 1.9 & 14.1 & 13.2 & 16.7 & 10.1 \\
\hline 2 & 6.1 & 1.1 & .3 & -.1 & -.4 & -.2 & .0 & 4.0 & 14.7 & 13.8 & 18.0 & 11.1 \\
\hline 3 & 5.8 & 1.1 & .3 & -.2 & -.4 & -.2 & .0 & 5.5 & 14.9 & 16.0 & 18.4 & 11.4 \\
\hline 4 & 5.3 & 1.0 & .3 & -.2 & -.5 & -.2 & .0 & 7.1 & 15.7 & 17.1 & 17.9 & 6.1 \\
\hline 5 & 5.3 & 1.0 & .3 & -.2 & -.6 & -.2 & .0 & 8.2 & 15.3 & 16.8 & 17.0 & 7.1 \\
\hline 6 & 5.3 & .9 & .2 & -.3 & -.6 & -.2 & .0 & 8.6 & 14.1 & 16.2 & 16.8 & 9.2 \\
\hline 7 & 5.4 & .9 & .2 & -.2 & -.6 & -.2 & .0 & 9.0 & 13.0 & 16.6 & 18.2 & 9.8 \\
\hline 8 & 5.2 & .9 & .2 & -.2 & -.5 & -.2 & .0 & 9.4 & 13.4 & 17.3 & 19.7 & 10.6 \\
\hline 9 & 5.3 & .7 & .2 & -.2 & -.4 & -.2 & .0 & 9.6 & 14.8 & 17.8 & 20.1 & 11.7 \\
\hline 10 & 5.3 & .7 & .2 & -.2 & -.4 & -.2 & .0 & 9.4 & 15.9 & 17.8 & 18.9 & 12.4 \\
\hline 11 & 5.7 & .8 & .2 & -.2 & -.4 & -.2 & .0 & 10.5 & 16.7 & 16.5 & 18.3 & 13.6 \\
\hline 12 & 6.9 & .8 & .2 & -.2 & -.4 & -.1 & .0 & 10.4 & 14.3 & 14.8 & 17.2 & 12.7 \\
\hline 13 & 5.8 & .7 & .2 & -.2 & -.3 & -.1 & .0 & 11.6 & 12.2 & 13.4 & 15.7 & 11.9 \\
\hline 14 & 5.2 & .6 & .2 & -.2 & -.3 & .0 & .0 & 11.9 & 9.9 & 13.7 & 15.7 & 12.2 \\
\hline 15 & 4.6 & .6 & .1 & -.2 & -.3 & .0 & .0 & 11.9 & 6.3 & 13.9 & 15.6 & 11.7 \\
\hline 16 & 5.1 & .5 & .1 & -.2 & -.3 & .0 & .0 & 11.7 & 4.4 & 14.4 & 15.8 & 12.0 \\
\hline 17 & 4.7 & .5 & .1 & -.2 & -.3 & .0 & .0 & 12.5 & 5.8 & 15.7 & 15.6 & 11.7 \\
\hline 18 & 4.5 & .5 & .1 & -.2 & -.3 & .0 & .0 & 12.7 & 9.2 & 15.3 & 15.9 & 10.2 \\
\hline 19 & 4.0 & .5 & .1 & -.2 & -.3 & .0 & .0 & 12.5 & 9.9 & 16.6 & 17.4 & 9.0 \\
\hline 20 & 3.4 & .5 & .1 & -.2 & -.3 & .0 & .0 & 10.8 & 11.3 & 17.6 & 17.2 & 9.6 \\
\hline 21 & 2.7 & .5 & .1 & -.2 & -.3 & .0 & .0 & 10.5 & 12.6 & 17.6 & 16.7 & 10.2 \\
\hline 22 & 3.5 & .5 & .1 & -.3 & -.3 & .0 & .0 & 10.5 & 14.5 & 18.3 & 15.2 & 11.3 \\
\hline 23 & 3.3 & .5 & .1 & -.3 & -.3 & .0 & .0 & 11.0 & 15.3 & 18.1 & 14.0 & 11.9 \\
\hline 24 & 1.5 & .4 & .1 & -.3 & -.2 & .0 & .0 & 12.1 & 15.0 & 18.5 & 13.9 & 11.2 \\
\hline 25 & 1.3 & .4 & .0 & -.4 & -.2 & .0 & .0 & 12.3 & 13.1 & 17.8 & 14.3 & 9.7 \\
\hline 26 & 1.5 & .4 & .0 & -.4 & -.2 & .0 & .0 & 13.2 & 14.0 & 17.1 & 14.4 & 9.0 \\
\hline 27 & 1.5 & .3 & .0 & -.4 & -.2 & .0 & .0 & 13.4 & 15.4 & 16.6 & 14.7 & 9.0 \\
\hline 28 & 1.5 & .3 & .0 & -.4 & -.2 & .0 & .0 & 13.1 & 16.4 & 17.3 & 15.1 & 9.1 \\
\hline 29 & 1.4 & .3 & .0 & -.4 & -.2 & .0 & .0 & 12.3 & 14.2 & 17.8 & 14.3 & 9.8 \\
\hline 30 & 1.3 & .3 & .0 & -.4 & $\ldots$ & .0 & .3 & 12.1 & 14.0 & 18.8 & 11.4 & 10.8 \\
\hline 31 & 1.3 & --- & .0 & -.4 & --- & .0 & --- & 13.0 & - & 17.5 & 10.9 & -- \\
\hline MEAN & 4.1 & .6 & .1 & -.3 & -.3 & -.1 & .0 & 10.4 & 13.0 & 16.4 & 16.2 & 10.5 \\
\hline $\operatorname{MAX}$ & 6.9 & 1.2 & .3 & -.1 & -.2 & .0 & .3 & 13.4 & 16.7 & 18.8 & 20.1 & 13.6 \\
\hline MIN & 1.3 & .3 & .0 & -.4 & -.6 & -.3 & .0 & 1.9 & 4.4 & 13.2 & 10.9 & 6.1 \\
\hline MED & 4.7 & .5 & .1 & -.2 & -.3 & .0 & .0 & 11.0 & 14.1 & 16.8 & 15.9 & 10.7 \\
\hline
\end{tabular}

CAL YR 1991 MEAN 4.9 MAX 21.2 MIN -3.8 MED $\quad .9$

WTR YR 1992 MEAN 5.9 MAX 20.1 MIN -.6 MED 1.3 
EAST STEWART BASIN

EAST STEWART CREEK NEAR IONE, NV

DAILY TOTAL SOLAR RADIATION, INCIDENTAL, WATER YEAR OCTOBER 1991 TO SEPTEMBER 1992 CALORIES PER SQUARE CENTIMETER

\begin{tabular}{|c|c|c|c|c|c|c|c|c|c|c|c|c|}
\hline DAY & OCT & NOV & DEC & JAN & FEB & MAR & APR & MAY & JUN & JUL & AUG & SEP \\
\hline 1 & 434 & 275 & 163 & 161 & 169 & 342 & 540 & 351 & 632 & 402 & 476 & 292 \\
\hline 2 & 426 & 261 & 180 & 166 & 247 & 179 & 557 & 455 & 549 & 732 & 539 & 410 \\
\hline 3 & 418 & 252 & 174 & 155 & 252 & 157 & 563 & 467 & 667 & 672 & 579 & 356 \\
\hline 4 & 417 & 253 & 174 & 161 & 256 & 361 & 458 & 525 & 731 & 724 & 448 & 349 \\
\hline 5 & 407 & 260 & 171 & 43 & 183 & 399 & 567 & 483 & 634 & 651 & 291 & 525 \\
\hline 6 & 350 & 256 & 175 & 18 & 137 & 385 & 564 & 433 & 327 & 555 & 375 & 562 \\
\hline 7 & 298 & 252 & 57 & 37 & 241 & 377 & 574 & 508 & 463 & 727 & 659 & 545 \\
\hline 8 & 394 & 155 & 34 & 146 & 264 & 162 & 535 & 516 & 467 & 586 & 665 & 521 \\
\hline 9 & 348 & 74 & 162 & 175 & 221 & 341 & 513 & 682 & 617 & 712 & 680 & 528 \\
\hline 10 & 380 & 149 & 165 & 173 & 247 & 442 & 405 & 686 & 692 & 559 & 335 & 471 \\
\hline 11 & 260 & 233 & 161 & 96 & 223 & 446 & 454 & 687 & 731 & 324 & 341 & 444 \\
\hline 12 & 306 & 207 & 157 & 188 & 147 & 456 & 373 & 512 & 388 & 391 & 204 & 520 \\
\hline 13 & 360 & 151 & 157 & 162 & 264 & 446 & 409 & 592 & 636 & 303 & 342 & 498 \\
\hline 14 & 362 & 85 & 146 & 187 & 315 & 368 & 262 & 616 & 60 & 388 & 341 & 507 \\
\hline 15 & 362 & 102 & 123 & 190 & 144 & 443 & 305 & 639 & 158 & 284 & 312 & 505 \\
\hline 16 & 356 & 218 & 156 & 175 & 208 & 247 & 490 & 544 & 460 & 524 & 418 & 485 \\
\hline 17 & 350 & 65 & 144 & 164 & 316 & 225 & 332 & 695 & 462 & 413 & 340 & 350 \\
\hline 18 & 348 & 108 & 158 & 200 & 257 & 475 & 636 & 625 & 576 & 474 & 379 & 95 \\
\hline 19 & 335 & 198 & 93 & 204 & 287 & 454 & 628 & 595 & 372 & 724 & 602 & 482 \\
\hline 20 & 338 & 85 & 157 & 208 & 306 & 320 & 532 & 394 & 497 & 724 & 613 & 474 \\
\hline 21 & 333 & 200 & 157 & 208 & 184 & 264 & 338 & 580 & 627 & 722 & 567 & 477 \\
\hline 22 & 229 & 200 & 145 & 213 & 245 & 272 & 599 & 542 & 688 & 691 & 583 & 465 \\
\hline 23 & 216 & 196 & 140 & 219 & 363 & 270 & 580 & 560 & 457 & 704 & 609 & 391 \\
\hline 24 & 177 & 163 & 152 & 181 & 360 & 450 & 590 & 630 & 570 & 704 & 527 & 460 \\
\hline 25 & 117 & 180 & 153 & 191 & 370 & 310 & 595 & 488 & 240 & 486 & 585 & 461 \\
\hline 26 & 84 & 177 & 159 & 148 & 379 & 395 & 482 & 633 & 497 & 433 & 597 & 456 \\
\hline 27 & 122 & 74 & 161 & 225 & 386 & 453 & 640 & 673 & 700 & 395 & 594 & 449 \\
\hline 28 & 290 & 165 & 150 & 78 & 377 & 370 & 628 & 364 & 612 & 478 & 524 & 446 \\
\hline 29 & 84 & 112 & 113 & 233 & 346 & 474 & 619 & 444 & 279 & 534 & 264 & 434 \\
\hline 30 & 297 & 173 & 151 & 232 & -- & 351 & 539 & 470 & 688 & 555 & 362 & 426 \\
\hline 31 & 292 & --- & 160 & 243 & --- & . 204 & --- & 664 & --- & 327 & 389 & -- \\
\hline TOTAL & 9490 & 5279 & 4548 & 5180 & 7694 & 10838 & 15307 & 17053 & 15477 & 16898 & 14540 & 13384 \\
\hline AL YR & 1991 & & & & & & & & & & & \\
\hline WTR YR & 1992 & TOTAL & 688 & & & & & & & & & \\
\hline
\end{tabular}


EAST STEWART BASIN

EAST STEWART CREEK NEAR IONE, NV

DAILY MEAN RELATIVE HUMIDITY, WATER YEAR OCTOBER 1991 TO SEPTEMBER 1992 PERCENT

\begin{tabular}{|c|c|c|c|c|c|c|c|c|c|c|c|c|}
\hline DAY & $O C T$ & Nov & DEC & JAN & FEB & MAR & APR & MAY & JUN & JUL & AUG & SEP \\
\hline 1 & 47 & 68 & 61 & 31 & 45 & 54 & 40 & 77 & 27 & 75 & 36 & 53 \\
\hline 2 & 37 & 81 & 53 & 25 & 47 & 96 & 30 & 38 & 35 & 39 & 32 & 30 \\
\hline 3 & 32 & 64 & 41 & 56 & 37 & 99 & 31 & 23 & 29 & 22 & 23 & 40 \\
\hline 4 & 22 & 65 & 32 & 59 & 62 & 83 & 32 & 23 & 29 & 19 & 22 & 83 \\
\hline 5 & 27 & 81 & 23 & 85 & 70 & 54 & 25 & 29 & 20 & 18 & 32 & 46 \\
\hline 6 & 23 & 80 & 21 & 96 & 96 & 81 & 29 & 60 & 26 & 20 & 39 & 28 \\
\hline 7 & 30 & 70 & 69 & 96 & 89 & 72 & 36 & 64 & 39 & 27 & 26 & 32 \\
\hline 8 & 28 & 54 & 61 & 61 & 75 & 94 & 34 & 53 & 41 & 26 & 17 & 26 \\
\hline 9 & 29 & 99 & 51 & 42 & 54 & 68 & 26 & 35 & 33 & 21 & 19 & 31 \\
\hline 10 & 25 & 73 & 63 & 22 & 81 & 48 & 39 & 27 & 23 & 29 & 19 & 18 \\
\hline 11 & 25 & 32 & 34 & 70 & 65 & 31 & 25 & 32 & 24 & 77 & 22 & 23 \\
\hline 12 & 38 & 32 & 56 & 65 & 77 & 33 & 20 & 30 & 47 & 86 & 44 & 31 \\
\hline 13 & 29 & 53 & 28 & 45 & 76 & 39 & 37 & 38 & 40 & 69 & 48 & 29 \\
\hline 14 & 21 & 93 & 23 & 66 & 54 & 41 & 80 & 38 & 100 & 68 & 49 & 19 \\
\hline 15 & 20 & 92 & 20 & 27 & 80 & 33 & 81 & 39 & 99 & 68 & 66 & 21 \\
\hline 16 & 22 & 47 & 22 & 35 & 93 & 73 & 62 & 24 & 84 & 42 & 65 & 35 \\
\hline 17 & 31 & 81 & 38 & 55 & 82 & 98 & 66 & 19 & 59 & 45 & 47 & 46 \\
\hline 18 & 36 & 98 & 64 & 33 & 71 & 69 & 50 & 20 & 40 & 44 & 36 & 92 \\
\hline 19 & 37 & 51 & 94 & 22 & 75 & 42 & 38 & 30 & 42 & 32 & 24 & 50 \\
\hline 20 & 48 & 67 & 27 & 25 & 65 & 51 & 25 & 49 & 38 & 26 & 27 & 45 \\
\hline 21 & 35 & 83 & 26 & 35 & 71 & 79 & 46 & 38 & 26 & 24 & 25 & 39 \\
\hline 22 & 38 & 24 & 36 & 24 & 83 & 84 & 34 & 27 & 17 & 20 & 22 & 33 \\
\hline 23 & 70 & 30 & 47 & 24 & 29 & 91 & 26 & 27 & 35 & 22 & 23 & 44 \\
\hline 24 & 49 & 53 & 40 & 22 & 34 & 63 & 22 & 20 & 41 & 21 & 20 & 35 \\
\hline 25 & 67 & 59 & 32 & 22 & 50 & 51 & 21 & 23 & 77 & 19 & 23 & 36 \\
\hline 26 & 96 & 27 & 24 & 28 & 24 & 63 & 22 & 35 & 73 & 21 & 19 & 26 \\
\hline 27 & 97 & 64 & 38 & 27 & 35 & 58 & 23 & 29 & 37 & 20 & 17 & 23 \\
\hline 28 & 95 & 94 & 71 & 46 & 32 & 60 & 24 & 22 & 29 & 18 & 18 & 21 \\
\hline 29 & 98 & 92 & 71 & 21 & 30 & 63 & 18 & 42 & 59 & 18 & 56 & 21 \\
\hline 30 & 75 & 82 & 82 & 20 & --- & 79 & 42 & 33 & 58 & 16 & 47 & 30 \\
\hline 31 & 52 & --- & 27 & 20 & -- & 82 & --- & 26 & --- & 29 & 67 & --- \\
\hline MEAN & 44 & 66 & 44 & 42 & 61 & 66 & 36 & 35 & 44 & 35 & 33 & 36 \\
\hline $\operatorname{MAX}$ & 98 & 99 & 94 & 96 & 96 & 99 & 81 & 77 & 100 & 86 & 67 & 92 \\
\hline MIN & 20 & 24 & 20 & 20 & 24 & 31 & 18 & 19 & 17 & 16 & 17 & 18 \\
\hline MED & 36 & 68 & 38 & 33 & 65 & 63 & 31 & 30 & 38 & 26 & 26 & 32 \\
\hline
\end{tabular}

CAL YR 1991 MEAN 47 MAX 99 MIN 15 MED 40 WTR YR 1992 MEAN 45 MAX 100 MIN 16 MED 38 
EAST STEWART BASIN

EAST STEWART CREEK NEAR IONE, NV

DAILY TOTAL PRECIPITATION, WATER YEAR OCTOBER 1991 TO SEPTEMBER 1992 INCHES

\begin{tabular}{|c|c|c|c|c|c|c|c|c|c|c|c|c|}
\hline DAY & OCT & NOV & $\mathrm{DEC}$ & JAN & FEB & MAR & APR & MAY & JUN & JUL & AUG & SEP \\
\hline 1 & .00 & .00 & .00 & .00 & .00 & .30 & .00 & .00 & .00 & .00 & .00 & .00 \\
\hline 2 & .00 & .00 & .00 & .00 & .00 & .20 & .00 & .00 & .00 & .00 & .00 & .00 \\
\hline 3 & .00 & .00 & .00 & .00 & .00 & .30 & .00 & .00 & .00 & .00 & .00 & 1.40 \\
\hline 4 & .00 & .00 & .00 & .00 & .00 & .20 & .00 & .00 & .00 & .00 & .00 & .11 \\
\hline 5 & .00 & .60 & .00 & 1.50 & .10 & .00 & .00 & .00 & .00 & .00 & .00 & .04 \\
\hline 6 & .00 & .10 & .00 & .20 & .50 & .35 & .00 & .00 & .00 & .00 & .00 & .00 \\
\hline 7 & .00 & .00 & .35 & .00 & .10 & .05 & .00 & .00 & .00 & .00 & .00 & .00 \\
\hline 8 & .00 & .00 & .00 & .00 & .00 & .00 & .00 & .10 & .00 & .00 & .00 & .00 \\
\hline 9 & .00 & .00 & .00 & .00 & .15 & .00 & .00 & .00 & .00 & .00 & .00 & .00 \\
\hline 10 & .00 & .30 & .00 & .00 & .35 & .00 & .00 & .00 & .00 & .00 & .00 & .00 \\
\hline 11 & .00 & .00 & .00 & .20 & .10 & .00 & .00 & .00 & .00 & .28 & .00 & .00 \\
\hline 12 & .00 & .00 & .00 & .00 & .20 & .00 & .00 & .00 & .08 & .00 & .08 & .00 \\
\hline 13 & .00 & .50 & .00 & .00 & .10 & .00 & .08 & .00 & .07 & .00 & .18 & .00 \\
\hline 14 & .00 & .00 & .00 & .00 & .05 & .00 & .12 & .00 & .45 & .00 & .02 & .00 \\
\hline 15 & .00 & .00 & .00 & .00 & .40 & .00 & .05 & .00 & .52 & .07 & .18 & .00 \\
\hline 16 & .00 & .00 & .00 & .00 & .25 & .10 & .00 & .00 & .18 & .00 & .02 & .00 \\
\hline 17 & .00 & .60 & .00 & .00 & .00 & .30 & .10 & .00 & .00 & .05 & .00 & .35 \\
\hline 18 & .00 & .00 & .30 & .00 & .05 & .00 & .00 & .00 & .00 & .00 & .00 & .15 \\
\hline 19 & .00 & .00 & .20 & .00 & .00 & .00 & .00 & .00 & .00 & .00 & .00 & .00 \\
\hline 20 & .00 & .00 & .00 & .00 & .00 & .00 & .00 & .00 & .00 & .00 & .00 & .00 \\
\hline 21 & .00 & .00 & .00 & .00 & .05 & .10 & .00 & .00 & .00 & .00 & .00 & .00 \\
\hline 22 & .00 & .00 & .00 & .00 & .20 & .30 & .00 & .00 & .00 & .00 & .00 & .00 \\
\hline 23 & .00 & .00 & .00 & .00 & .00 & .10 & .00 & .00 & .00 & .00 & .00 & .00 \\
\hline 24 & .00 & .00 & .00 & .00 & .00 & .00 & .00 & .00 & .00 & .00 & .00 & .00 \\
\hline 25 & .10 & .00 & .00 & .00 & .00 & .00 & .00 & .00 & .15 & .00 & .00 & .00 \\
\hline 26 & 1.10 & .00 & .00 & .00 & .00 & .00 & .00 & .00 & .00 & .00 & .00 & .00 \\
\hline 27 & .10 & .40 & .00 & .00 & .00 & .00 & .00 & .00 & .00 & .00 & .00 & .00 \\
\hline 28 & .90 & .10 & .20 & .10 & .00 & .00 & .00 & .00 & .05 & .00 & .00 & .00 \\
\hline 29 & .00 & .25 & .10 & .00 & .00 & .10 & .00 & .00 & .00 & .00 & .22 & .00 \\
\hline 30 & .00 & .00 & .00 & .00 & -- & .20 & .10 & .00 & .10 & .00 & .00 & .00 \\
\hline 31 & .00 & -- & .00 & .00 & -- & .10 & --- & .00 & --- & .04 & .15 & -- \\
\hline TOTAL & 2.20 & 2.85 & 1.15 & 2.00 & 2.60 & 2.70 & 0.45 & 0.10 & 1.60 & 0.44 & 0.85 & 2.05 \\
\hline
\end{tabular}

CAL YR 1991 TOTAL 23.64

WTR YR 1992 TOTAL 18.99 
EAST STEWART CREEK BASIN

EAST STEWART CREEK NEAR IONE, NV PRECIPITATION WATER QUALITY, WATER YEAR OCTOBER 1991 TO SEPTEMBER 1992

\begin{tabular}{|c|c|c|c|c|c|c|c|c|c|c|c|}
\hline DATE & TIME & $\begin{array}{l}\text { SPE- } \\
\text { CIFIC } \\
\text { CON- } \\
\text { DUCT- } \\
\text { ANCE } \\
\text { (US/CM) }\end{array}$ & $\begin{array}{c}\text { SPE- } \\
\text { CIFIC } \\
\text { CON- } \\
\text { DUCT- } \\
\text { ANCE } \\
\text { LAB } \\
\text { (US/CM) }\end{array}$ & $\begin{array}{c}\text { PH } \\
\text { WATER } \\
\text { WHOLE } \\
\text { FIELD } \\
\text { (STAND- } \\
\text { ARD } \\
\text { UNITS) }\end{array}$ & $\begin{array}{c}\text { PH } \\
\text { WATER } \\
\text { WHOLE } \\
\text { LAB } \\
\text { (STAND- } \\
\text { ARD } \\
\text { UNITS) }\end{array}$ & $\begin{array}{c}\text { ALKA- } \\
\text { LINITY } \\
\text { LAB } \\
\text { (MG/L } \\
\text { AS } \\
\text { CACO3) }\end{array}$ & $\begin{array}{l}\text { CALCIUM } \\
\text { DIS- } \\
\text { SOLVED } \\
\text { (MG/L } \\
\text { AS CA) }\end{array}$ & $\begin{array}{l}\text { MAGNE- } \\
\text { SIUM, } \\
\text { DIS- } \\
\text { SOLVED } \\
\text { (MG/L } \\
\text { AS MG) }\end{array}$ & $\begin{array}{c}\text { SODIUM, } \\
\text { DIS- } \\
\text { SOLVED } \\
\text { (MG/L } \\
\text { AS NA) }\end{array}$ & $\begin{array}{l}\text { POTAS- } \\
\text { SIUM, } \\
\text { DIS- } \\
\text { SOLVED } \\
\text { (MG/L } \\
\text { AS K) }\end{array}$ & $\begin{array}{c}\text { SULFATE } \\
\text { DIS- } \\
\text { SOLVED } \\
\text { (MG/L } \\
\text { AS SO4) }\end{array}$ \\
\hline $\begin{array}{c}\text { FEB } 1992 \\
19 \ldots\end{array}$ & 1100 & 9 & 10 & 6.6 & 6.5 & 2.6 & 0.73 & 0.17 & $<0.20$ & 1.1 & 0.26 \\
\hline $\begin{array}{l}A P R \\
09 \ldots \\
\text { JUN }\end{array}$ & 1045 & 33 & 6 & 7.1 & 6.1 & $<0.5$ & 0.20 & 0.02 & $<0.20$ & $<0.10$ & 0.60 \\
\hline${ }_{\text {JUL }}^{11 \ldots}$ & 1135 & 36 & 38 & 6.3 & 7.1 & 6.1 & 2.4 & 0.23 & 2.0 & 0.70 & 3.4 \\
\hline $\begin{array}{l}29 . . . \\
\text { SEP }\end{array}$ & 0915 & 23 & 21 & 6.2 & 5.7 & 5.7 & 0.90 & 0.21 & $<0.20$ & 3.7 & 0.93 \\
\hline $16 \ldots$ & 1100 & 12 & 10 & 5.9 & 6.2 & 0.7 & 0.63 & 0.06 & $<0.20$ & 0.20 & 1.1 \\
\hline DATE & $\begin{array}{l}\text { CHLO- } \\
\text { RIDE, } \\
\text { DIS- } \\
\text { SOLVED } \\
\text { (MG/L } \\
\text { AS CL) }\end{array}$ & $\begin{array}{l}\text { FLUO- } \\
\text { RIDE, } \\
\text { DIS- } \\
\text { SOLVED } \\
\text { (MG/L } \\
\text { AS F) }\end{array}$ & $\begin{array}{c}\text { BROMIDE } \\
\text { DIS- } \\
\text { SOLVED } \\
\text { (MG/L } \\
\text { AS BR) }\end{array}$ & $\begin{array}{c}\text { SILICA, } \\
\text { DIS- } \\
\text { SOLVED } \\
\text { (MG/L } \\
\text { AS } \\
\text { SIO2) }\end{array}$ & $\begin{array}{c}\text { NITRO- } \\
\text { GEN, } \\
\text { NITRATE } \\
\text { DIS- } \\
\text { SOLVED } \\
\text { (MG/L } \\
\text { AS N) }\end{array}$ & $\begin{array}{l}\text { PHOS- } \\
\text { PHORUS } \\
\text { ORTHO, } \\
\text { DIS- } \\
\text { SOLVED } \\
\text { (MG/L } \\
\text { AS P) }\end{array}$ & $\begin{array}{l}\text { IRON, } \\
\text { DIS- } \\
\text { SOLVED } \\
\text { (UG/L } \\
\text { AS FE) }\end{array}$ & $\begin{array}{l}\text { MANGA- } \\
\text { NESE, } \\
\text { DIS- } \\
\text { SOLVED } \\
\text { (UG/L } \\
\text { AS MN) }\end{array}$ & $\begin{array}{c}\text { H-2 / } \\
\text { H-1 } \\
\text { STABLE } \\
\text { ISOTOPE } \\
\text { RATIO } \\
\text { PER } \\
\text { MIL }\end{array}$ & $\begin{array}{c}\text { O-18 } \\
\text { O-16 } \\
\text { STABLE } \\
\text { ISOTOPE } \\
\text { RATIO } \\
\text { PER } \\
\text { MIL }\end{array}$ & \\
\hline $\begin{array}{c}\text { FEB } 1992 \\
19 \ldots \\
\text { APR }\end{array}$ & 0.43 & 0.09 & $<0.010$ & $<0.10$ & 0.010 & $<0.010$ & 8 & 15 & -118.0 & -16.50 & \\
\hline $\begin{array}{c}09 . . \\
\text { JUN }\end{array}$ & 0.07 & $<0.01$ & $<0.010$ & $<0.10$ & 0.230 & $<0.010$ & 8 & 2 & -109.0 & -15.10 & \\
\hline${ }_{\text {JuL }}^{11 \ldots}$ & 1.1 & 0.16 & $<0.010$ & $<0.10$ & 1.20 & $<0.010$ & $<3$ & 19 & - & -- & \\
\hline $\operatorname{SEP}^{29} \cdots$ & 0.31 & 0.13 & 0.040 & $<0.10$ & $<0.010$ & 0.220 & $<3$ & 12 & -111.0 & -15.70 & \\
\hline $16 \ldots$ & 0.22 & 0.07 & $<0.010$ & $<0.10$ & 0.710 & $<0.010$ & $<3$ & 11 & -85.0 & -12.05 & \\
\hline
\end{tabular}


EAST STEWART CREEK BASIN

EAST STEWART CREEK NEAR IONE, NV

WATER-QUALITY ANALYSIS FROM THE UNSATURATED-ZONE LYSIMETER, WATER YEAR OCTOBER 1991 TO SEPTEMBER 1992

\begin{tabular}{|c|c|c|c|c|c|c|c|c|}
\hline & IME & $\begin{array}{c}\text { SPE- } \\
\text { CIFIC } \\
\text { CON- } \\
\text { DUCT- } \\
\text { ANCE } \\
\text { LAB } \\
\text { (US/CM) }\end{array}$ & $\begin{array}{c}\text { PH } \\
\text { WATER } \\
\text { WHOLE } \\
\text { LABB } \\
\text { (STAND- } \\
\text { ARD } \\
\text { UNITS) }\end{array}$ & $\begin{array}{c}\text { CALCIUM } \\
\text { DIS- } \\
\text { SOLVED } \\
\text { (MG/L } \\
\text { AS CA) }\end{array}$ & $\begin{array}{l}\text { MAGNE- } \\
\text { SIUM, } \\
\text { DIS- } \\
\text { SOLVED } \\
\text { (MG/L } \\
\text { AS MG) }\end{array}$ & $\begin{array}{l}\text { SODIUM, } \\
\text { DIS- } \\
\text { SOLVED } \\
\text { (MG/L } \\
\text { AS NA) }\end{array}$ & $\begin{array}{l}\text { SULFATE } \\
\text { DIS- } \\
\text { SOLVED } \\
\text { (MG/L } \\
\text { AS SO4) }\end{array}$ & $\begin{array}{l}\text { CHLO- } \\
\text { RIDE, } \\
\text { DIS- } \\
\text { SOLVED } \\
\text { (MG/L } \\
\text { AS CL) }\end{array}$ \\
\hline
\end{tabular}

JUN 1992

$11 \ldots$

1430

130

7.3

9.6

1.9

5.9

2.2

1.4

DATE

CLUO-

FLUO-

RIDE,

BROMIDE
DIS-
SOLVED
(MG/L
AS BR)

SILICA,
DIS-
SOLVED
(MG/L
AS
SIO)

IRON,
DIS-
SOLVED
(UG/L
AS FE)

MANGA- $\begin{array}{cc}\mathrm{H}-2 \\ \mathrm{H}-1\end{array} \mid \begin{aligned} & 0-18 \\ & 0-16\end{aligned}$

NESE, STABLE

O-16

SOLVED

AS F)

AS BR)

SIO2)

AS FE)

DIS-

ISOTOPE ISOTOPE

SOLVED RATIO RATIO

(UG/L PER $\quad$ PER

JUN 1992

$11 .$. .

$<0.10$

0.020

8.3

18

$3 \quad-90.0$

$-12.05$ 
EAST STEWART BASIN

EAST STEWART CREEK NEAR IONE, NV

DAILY MEAN DISCHARGE, WATER YEAR OCTOBER 1991 TO SEPTEMBER 1992 CUBIC FEET PER SECOND

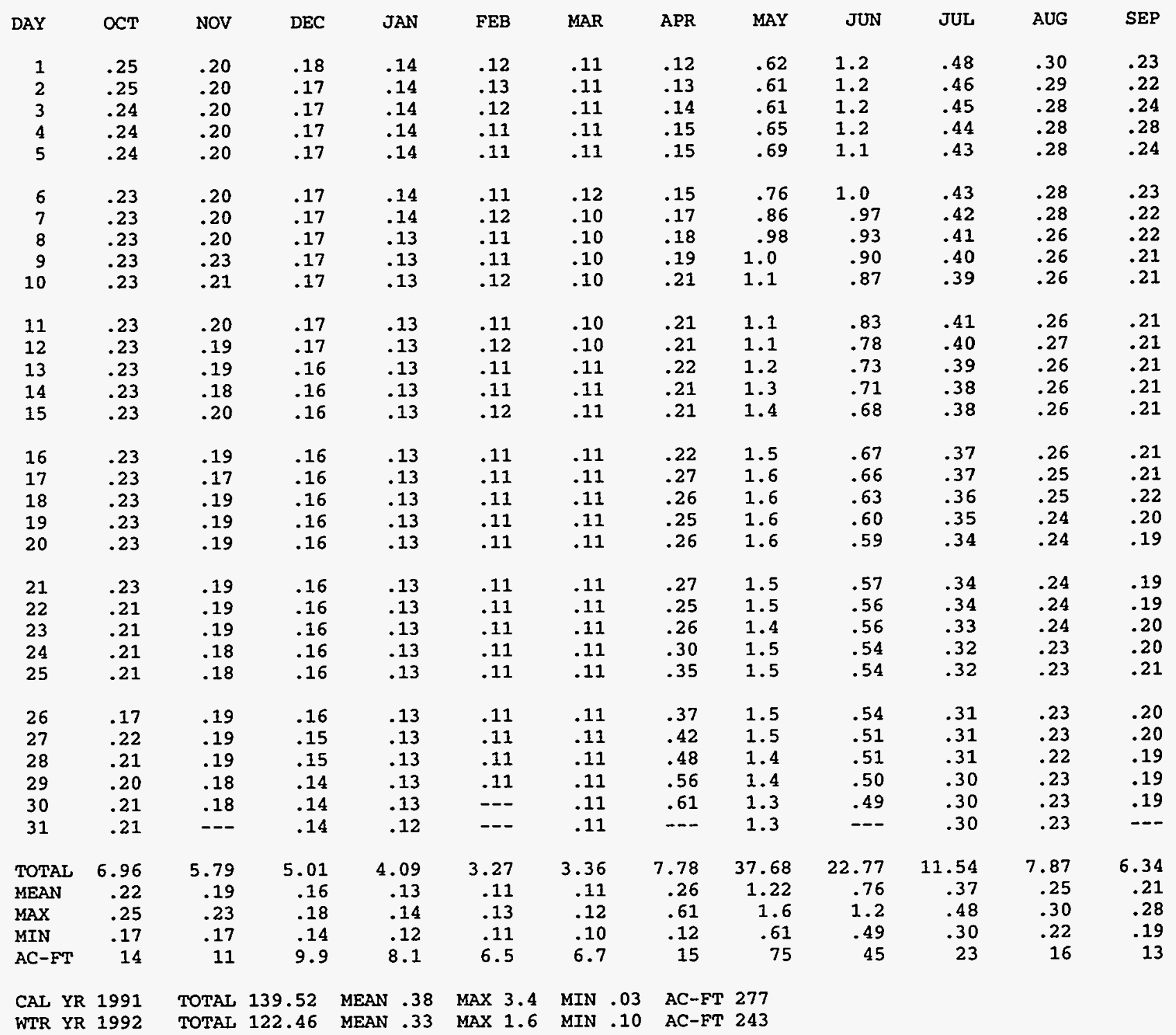


EAST STEWART CREEK BASIN

EAST STEWART CREEK NEAR IONE, NV

SURFACE-WATER QUALITY, WATER YEAR OCTOBER 1991 TO SEPTEMBER 1992

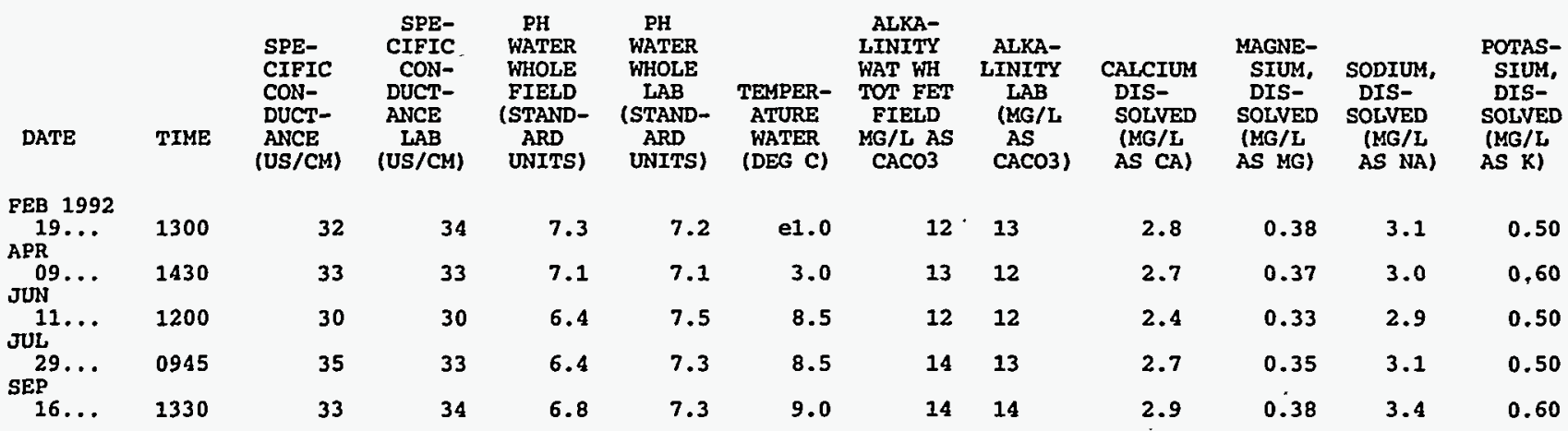

\begin{tabular}{|c|c|c|c|c|c|c|c|c|c|c|c|}
\hline DATE & $\begin{array}{l}\text { SULFATE } \\
\text { DIS- } \\
\text { SOLVED } \\
\text { (MG/L } \\
\text { AS SO4) }\end{array}$ & $\begin{array}{l}\text { CHLO- } \\
\text { RIDE, } \\
\text { DIS- } \\
\text { SOLVED } \\
\text { (MG/L } \\
\text { AS CL) }\end{array}$ & $\begin{array}{l}\text { FLUO- } \\
\text { RIDE, } \\
\text { DIS- } \\
\text { SOLVED } \\
\text { (MG/L } \\
\text { AS F) }\end{array}$ & $\begin{array}{c}\text { BROMIDE } \\
\text { DIS- } \\
\text { SOLVED } \\
\text { (MG/L } \\
\text { AS BR) }\end{array}$ & $\begin{array}{l}\text { SILICA, } \\
\text { DIS- } \\
\text { SOLVED } \\
\text { (MG/L } \\
\text { AS } \\
\text { SIO2) }\end{array}$ & $\begin{array}{c}\text { NITRO- } \\
\text { GEN, } \\
\text { NITRATE } \\
\text { DIS- } \\
\text { SOLVED } \\
\text { (KG/L } \\
\text { AS N) }\end{array}$ & $\begin{array}{c}\text { PHOS- } \\
\text { PHORUS } \\
\text { ORTHO, } \\
\text { DIS- } \\
\text { SOLVED } \\
\text { (MG/L } \\
\text { AS P) }\end{array}$ & $\begin{array}{l}\text { IRON, } \\
\text { DIS- } \\
\text { SOLVED } \\
\text { (UG/L } \\
\text { AS FE) }\end{array}$ & $\begin{array}{l}\text { MANGA- } \\
\text { NESE, } \\
\text { DIS- } \\
\text { SOLVED } \\
\text { (UG/L } \\
\text { AS NN) }\end{array}$ & $\begin{array}{c}\text { H-2 / } \\
\text { H-1 } \\
\text { STABLE } \\
\text { ISOTOPE } \\
\text { RATIO } \\
\text { PER } \\
\text { MIL }\end{array}$ & $\begin{array}{c}0-18 \text {, } \\
0-16 \\
\text { STABLE } \\
\text { ISOTOPE } \\
\text { RATIO } \\
\text { PER } \\
\text { MIL }\end{array}$ \\
\hline $\begin{array}{l}992 \\
\cdots\end{array}$ & 1.4 & 0.77 & 0.09 & $<0.010$ & 16 & $<0.010$ & $<0.010$ & 7 & 3 & -119.0 & -16.35 \\
\hline & 1.6 & 0.80 & 0.08 & $<0.010$ & 15 & 0.230 & 0.020 & 4 & $<1$ & -120.0 & -16.30 \\
\hline . & 1.3 & 0.56 & $<0.01$ & $<0.010$ & 16 & 0.190 & $<0.010$ & $<3$ & $<1$ & -119.0 & -16.25 \\
\hline & 1.3 & 0.62 & 0.07 & $<0.010$ & 16 & 0.100 & $<0.010$ & 4 & $<1$ & -122.0 & -16.35 \\
\hline & 1.3 & 0.71 & 0.06 & $<0.010$ & 18 & 0.030 & $<0.010$ & 4 & $<1$ & -118.0 & -16.30 \\
\hline
\end{tabular}


LOCATION. -- Lat $38^{\circ} 53^{\prime} 24^{\circ}$, Iong $117^{\circ} 21^{\prime} 36^{\prime}$, Nye County, Hydrologic Unit 16040107, 250 ft northeast of the East Stewart Creek surface-water equipment, $1.2 \mathrm{mi}$ southeast of the Columbine campground, and $13.3 \mathrm{mi}$ east of Ione on State Route 21 . PERIOD OF RECORD. -- MaY 1986 to current year. SURFACE-WATER QUALITY, WATER YEAR OCTOBER 1991 TO SEPTEMBER 1992

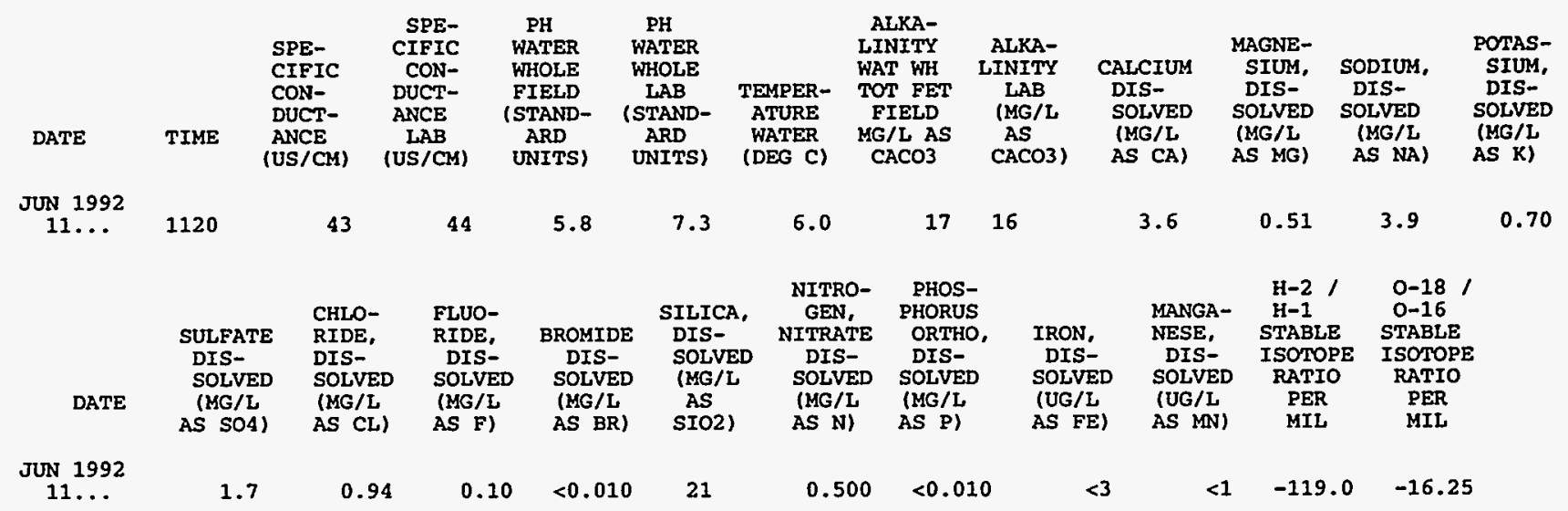

